\title{
BUILDING 579 WASTE ION EXCHANGE FACILITY CHARACTERIZATION REPORT
}

\section{ARGONNE NATIONAL LABORATORY ARGONNE, ILLINOIS}

\author{
Prepared by \\ C. M. Sholeen \\ D. C. Geraghty \\ Environment Safety and Health Division \\ Health Physics Section \\ Argonne National Laboratory \\ Argonne, Il 60439
}

DRAFT REPORT

March 1997 


\section{DISCLAMMER}

Portions of this document may be illegible in electronic image products. Images are produced from the best available original document. 


\title{
BUILDING 579 WASTE ION EXCHANGE FACILITY CHARACTERIZATION REPORT
}

\section{ARGONNE NATIONAL LABORATORY ARGONNE, ILLINOIS}

\author{
March 1997 \\ Characterization Performed and Report Prepared by \\ Health Physics Section \\ Environment, Safety and Health Division \\ Argonne National Laboratory
}

Site Preparation, Characterization and Report Team:

Charlotte Sholeen

Dolores Geraghty

Nick Contos

Timothy Branch

Joseph Cooney

Sue Santarelli

Cindy Sullivan

Keith Dunn

David Reilly

Willis Ray

Consultant:

Judd Johnson 



\section{TABLE OF CONTENTS}

LIST OF TABLES $\ldots \ldots \ldots \ldots \ldots \ldots \ldots \ldots \ldots \ldots \ldots \ldots \ldots \ldots \ldots \ldots \ldots$

LIST OF FIGURES $\ldots \ldots \ldots \ldots \ldots \ldots \ldots \ldots \ldots \ldots \ldots \ldots \ldots \ldots \ldots \ldots \ldots$

ACKNOWLEDGMENTS $\ldots \ldots \ldots \ldots \ldots \ldots \ldots \ldots \ldots \ldots \ldots \ldots \ldots \ldots \ldots \ldots$ vii

ABSTRACT $\ldots \ldots \ldots \ldots \ldots \ldots \ldots \ldots \ldots \ldots \ldots \ldots \ldots \ldots \ldots \ldots \ldots \ldots$ viii

I. OBJECTIVE $\ldots \ldots \ldots \ldots \ldots \ldots \ldots \ldots \ldots \ldots \ldots \ldots \ldots \ldots \ldots \ldots \ldots$

II. HISTORY OF THE WASTE ION EXCHANGE FACILITY $\ldots \ldots \ldots \ldots \ldots$

III. CHARACTERIZATION METHODOLOGY $\ldots \ldots \ldots \ldots \ldots \ldots \ldots \ldots \ldots$

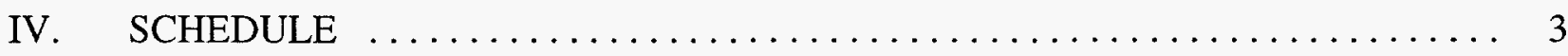

V. QUALITY ASSURANCE $\ldots \ldots \ldots \ldots \ldots \ldots \ldots \ldots \ldots \ldots \ldots \ldots \ldots \ldots$

VI. SAMPLES AND MEASUREMENTS IDENTIFICATION SYSTEM $\ldots \ldots \ldots \ldots 4$

VII. RELEASE CRITERIA AND BACKGROUND VALUES $\ldots \ldots \ldots \ldots \ldots \ldots$

VIII. INSTRUMENTATION $\ldots \ldots \ldots \ldots \ldots \ldots \ldots \ldots \ldots \ldots \ldots \ldots \ldots \ldots \ldots \ldots$

IX. NUMBER OF MEASUREMENTS AND SAMPLES $\ldots \ldots \ldots \ldots \ldots \ldots \ldots \ldots$

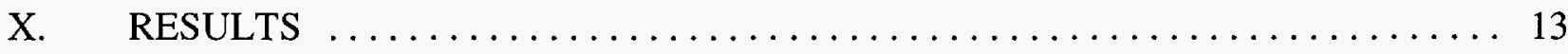

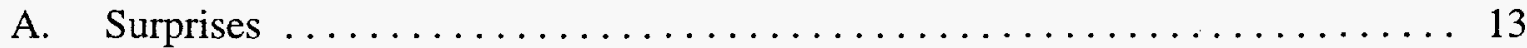

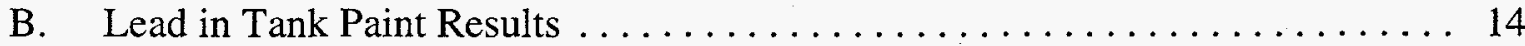

C. Asbestos Results . . . . . . . . . . . . . . . . . . . . . . . . . 14

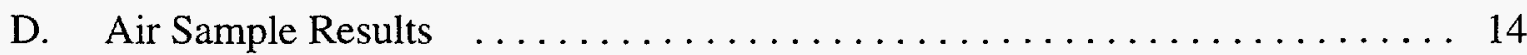

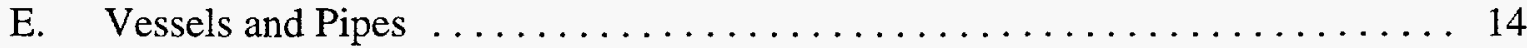

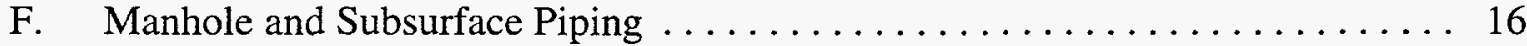

G. Building Interior, Trench and Pit $\ldots \ldots \ldots \ldots \ldots \ldots \ldots \ldots \ldots \ldots \ldots \ldots \ldots \ldots$

XI. SUMMARY OF RESULTS ABOVE BACKGROUND $\ldots \ldots \ldots \ldots \ldots \ldots \ldots$

XII. ESTIMATE OF INVENTORY OF ACTIVITY IN COLUMNS $\ldots \ldots \ldots \ldots \ldots$

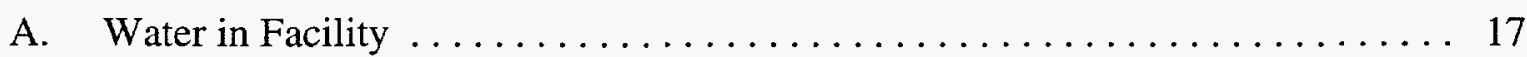

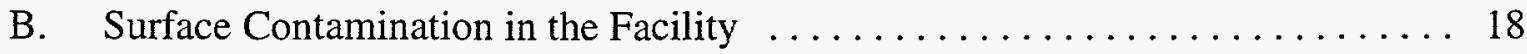

XIII. LESSONS LEARNED $\ldots \ldots \ldots \ldots \ldots \ldots \ldots \ldots \ldots \ldots \ldots \ldots \ldots \ldots \ldots$ 


\section{TABLE OF CONTENTS (Cont.)}

XIV. REFERENCES $\ldots \ldots \ldots \ldots \ldots \ldots \ldots \ldots \ldots \ldots \ldots \ldots \ldots \ldots \ldots \ldots \ldots$

APPENDIX A: Documentation of Samples $\ldots \ldots \ldots \ldots \ldots \ldots \ldots \ldots \ldots \ldots \ldots \ldots$

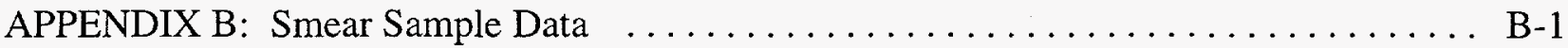

APPENDIX C: Air and Water Sample Data $\ldots \ldots \ldots \ldots \ldots \ldots \ldots \ldots \ldots \ldots \ldots$

APPENDIX D: Analytical Results $\ldots \ldots \ldots \ldots \ldots \ldots \ldots \ldots \ldots \ldots \ldots \ldots \ldots \ldots \ldots$

APPENDIX E: Instrument Calibration and Operational Check Records $\ldots \ldots \ldots \ldots \ldots$ E-1 


\section{LIST OF TABLES}

$1 \quad$ Allowable Residual Surface Contamination Limits $\ldots \ldots \ldots \ldots \ldots \ldots \ldots$

2 Typical Background Concentrations in Soil and Sediments $\ldots \ldots \ldots \ldots \ldots \ldots$

3 Instruments Used for Characterization $\ldots \ldots \ldots \ldots \ldots \ldots \ldots \ldots \ldots \ldots \ldots \ldots$

$4 \quad$ Measurements \& Samples Required for Retention Tank Characterization . . . . . . . 13

$5 \quad$ Gross $\alpha$ and $\beta$ Activity in Water Samples $\ldots \ldots \ldots \ldots \ldots \ldots \ldots \ldots \ldots \ldots \ldots$

$6 \quad$ Exchange Facility Water \& Resin Samples dated $9 / 20 / 96 \ldots \ldots \ldots \ldots \ldots \ldots$

$7 \quad$ Volumes in the Vessels, Tanks \& Manhole $\ldots \ldots \ldots \ldots \ldots \ldots \ldots \ldots \ldots \ldots$

$8 \quad$ RCRA Metal Contamination in the Cation Exchange Vessel .............. 19

9 Radioactive Contamination in the Cation Exchange Vessel \& Manhole . . . . . . 19

A1 Building 579 Characterization Sample Labels $\ldots \ldots \ldots \ldots \ldots \ldots \ldots$ A-4

B1 Composite Smear and Scraping Data for Building $579 \ldots \ldots \ldots \ldots \ldots \ldots$ B-3

B2 Smear Scraping and Water Data from Tennelec for Building $579 \ldots \ldots \ldots \ldots$ B-5

B3 Building 579 Smear Data logged on the DABRAS or NE $\ldots \ldots \ldots \ldots \ldots$ B-6

C1 Air Sample Data from Tennelec for Building $579 \ldots \ldots \ldots \ldots \ldots \ldots \ldots \ldots$ C-3

C2 Building 579 Characterization Water Samples $\ldots \ldots \ldots \ldots \ldots \ldots \ldots \ldots \ldots \ldots$

D1 Documents Containing Analytical Results $\ldots \ldots \ldots \ldots \ldots \ldots \ldots \ldots \ldots \ldots \ldots \ldots$

D2 Water and Resin from Exchange Vessels $\ldots \ldots \ldots \ldots \ldots \ldots \ldots \ldots \ldots \ldots \ldots \ldots \ldots$

E1 Source Data from Tennelec for Building $579 \ldots \ldots \ldots \ldots \ldots \ldots \ldots \ldots \ldots$ E-3

E2 Blank Data from Tennelec for Building $579 \ldots \ldots \ldots \ldots \ldots \ldots \ldots \ldots \ldots$ 


\section{LIST OF FIGURES}

$1 \quad$ Building 579 Piping to Adjacent Facilities

2 Building 579 Ion Exchange Piping \& Equipment $\ldots \ldots \ldots \ldots \ldots \ldots$

3 Retrospective Air Sampling System (Giraffe) . . . . . . . . . . . . . . . 9

$4 \quad$ NE Technology Model Electra with $100 \mathrm{~cm}^{2}$ Detector for

Measuring $\alpha \beta \gamma$ Surface Contamination . . . . . . . . . . . . . . . 11

$5 \quad$ Tennelec APC MII Smear Counting System $\ldots \ldots \ldots \ldots \ldots \ldots \ldots \ldots \ldots \ldots$

6 Dual Alpha Beta Radioactivity Assay System for smear counting . . . . . . . . 12

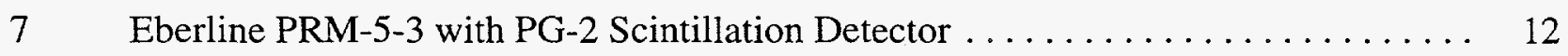

A1 Sample Labels $\ldots \ldots \ldots \ldots \ldots \ldots \ldots \ldots \ldots \ldots \ldots \ldots \ldots \ldots \ldots$

E1 Calibration Check Data for the Tennelec System $\ldots \ldots \ldots \ldots \ldots \ldots \ldots$

E2 Background Check Data for the Tennelec System $\ldots \ldots \ldots \ldots \ldots \ldots \ldots$ 


\section{ACKNOWLEDGMENTS}

Special thanks to Cindy Sullivan (ESH-HP) who compiled the draft document. The draft of the report could not have been produced on time without her dedication and efficiency.

Thanks to Tim Branch and Joe Cooney (ESH-HP) for the survey and sampling efforts. Thanks to Dolores Geraghty for coordinating the paper work, supervising the characterization effort and insuring the job was completed in a timely manner. Thanks to Nick Contos and Dave Reilly (ESH-HP) for generating the CAD drawings for the report.

Thanks to Sue Santarelli, Willis Ray and Keith Dunn (EMO-WM) for the use of equipment and the removal of the bolts from the tanks that permitted sample collection. Thanks to Jud Johnson (WMO-WM) for reviewing this document and recommending the TCLP analysis for the resin sample.

This effort is supported by the Department of Energy EM-40 Office in conjunction with the Technology Development Division of Argonne National Laboratory. 


\begin{abstract}
External direct surveys were performed for elevated $\gamma$ levels with a PG2 portable detector connected to a PRM 5-3 meter and for elevated $\alpha$ and $\beta$ levels with an NE portable detector. No $\gamma$ activity above background was detected. Several locations, the floor and west wall of building 579 and the manhole, had low levels of $\beta$ activity, up to $87 \pm 49 \mathrm{dis} / \mathrm{min}$. These values are below the allowable residual surface contamination limits for removable beta activity.

There is water in the Mixed Bed Exchange Vessel, the Cation Exchange Vessel, the Closed Drain Tank, the manhole and some of the pipes. The accessible internal surfaces of the pipes, tanks and columns had higher levels of $\beta$ activity up to $172 \pm 52 \mathrm{dis} / \mathrm{min}$ and some $\alpha$ activity up to $106 \pm$ $29 \mathrm{dis} / \mathrm{min}$. After the water is removed from the vessels, tanks, and lines, they should be surveyed to determine whether the areas accessible for smear surveys are representative of the general inside contamination levels.

There are elevated levels of radionuclides in the resin from the Cation Exchange Vessel and in the water from the manhole. Since the radionuclide concentrations in the manhole water are less than ten times the site release criteria, it does not need any processing before it is released to the onsite drains. Although there are RCRA metals on the resin in the Cation Exchange Vessel, the amount that is removed during a leaching analysis is below the toxicity Characteristic level. Therefore, the resin is a radioactive waste not a mixed waste.
\end{abstract}




\section{BUILDING 579 WASTE ION EXCHANGE FACILITY CHARACTERIZATION REPORT}

\section{OBJECTIVE}

The two primary goals to be achieved by a characterization are:

- Collection of enough data on the amount of contamination present to allow realistic cost estimates for radioactive and otherwise contaminated waste disposal.

- Identification of the extent of contaminated materials, areas, and equipment, to allow optimum task planning for the eventual Decontamination and Decommissioning $(\mathrm{D} \& \mathrm{D})$ process.

The 579 Waste Ion Exchange Facility has been slated for a D\&D Project leading to complete decontamination and full release for unrestricted use. Thus, the identification of radioactivity levels and hazardous materials levels above free release levels is critical for accurate D\&D planning.

\section{HISTORY OF THE WASTE ION EXCHANGE FACILITY}

The Waste Ion Exchange Facility was used to process waste fluids from a collecting lagoon. It was constructed in the 1950's, and is now non-functional. The ion exchange equipment was connected to the collecting lagoon, equalization tanks, and building 575 distribution pit by underground piping (see Figure 1). There are also a pad and influent lines on the south side of the building and another pad and effluent lines on the north side of the building.

\section{CHARACTERIZATION METHODOLOGY}

The general approach of the characterization can be summarized by the following steps:

- took samples for airborne radioactive particulates,

- thoroughly alpha and beta scanned the floor and walls to look for localized regions or spots with elevated radiation levels, 
March 19, 1997

Page 2

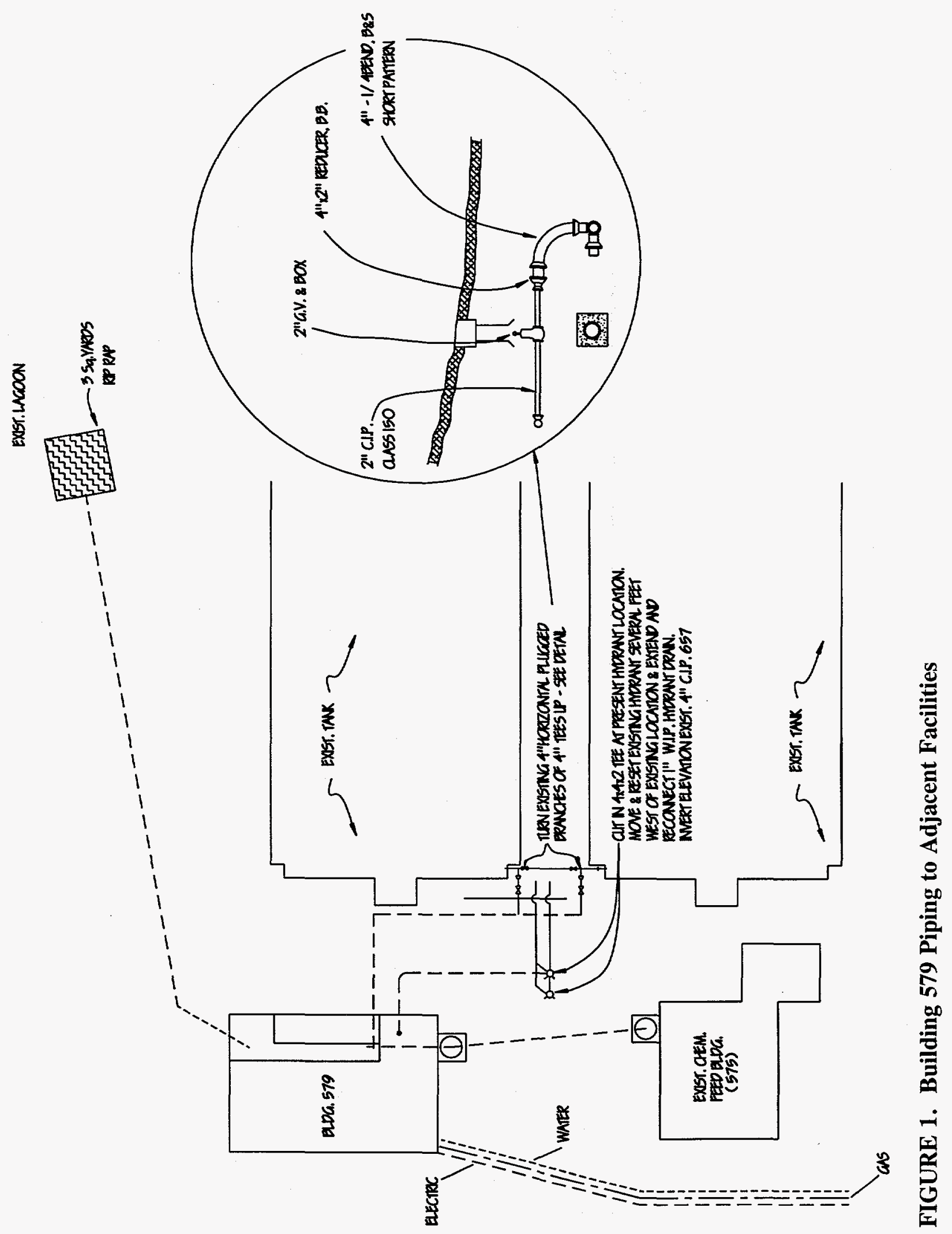


- gamma scanned the tanks and pipes to measure maximum radiation exposure rates,

- took large area smear samples at randomly selected locations on walls, floors, pipes and tanks.

- took smear samples from inside the tanks to determine removable $\alpha$ and $\beta \gamma$ activities,

- took samples of insulation on the pipes in the trench and tested for asbestos,

- took smears or water samples at preselected locations to assess the internal contamination of the lines,

- took water and resin samples from inside the exchange vessels and closed drain tank to analyze for RCRA metals, volatile organic compounds, semivolatile organic compounds, and radioactive contaminants.

Samples were pre-numbered and labels for the sample envelopes or plastic bags were pre-printed. An example of a sheet of sample labels is given in Appendix A. The samples were checked off when completed and also when analyzed for gross $\alpha$ and $\beta$ activity. Full lists of sample results are given in Appendix B for smear, Q-tip and scraping samples; Appendix C for air and water samples; and Appendix D for samples sent to the Control Laboratory (ESH-DA), or Argonne's Analytical Chemistry Laboratory (ACL) for analysis.

\section{SCHEDULE}

The Health Physics Section of the ANL-E Environment Safety and Health Division began characterization field work on September 12, 1996 and completed field work on September 25, 1996. The water and resin in the exchange vessels were sampled on September 20, 1996. Analyses of the water samples were completed on October 29, 1996. Analysis of the resin sample was completed on December 18, 1996. On February 6, 1997 a leachate analysis was requested because of the high level of metals in the resin. The leachate analysis was completed on February 25, 1997. 


\section{QUALITY ASSURANCE}

The Quality of the characterization process was assured by the following actions:

- All work was performed in compliance to the QA plans of the ESH Division, and the Health Physics Section.

- Calibration and Operational Checks - all instrumentation used in the characterization surveys were checked daily for proper operation. A log was maintained for operational checks. All radiation detection instrumentation were calibrated with NIST traceable radioactive sources. Instruments' operational check and calibration records are given in Appendix F.

- Data Protection - all data stored on computers were backed up daily.

- Special attention was given to contamination control during sampling of potentially contaminated materials to assure that the characterization process did not alter or bias the true condition of the facility.

\section{SAMPLES AND MEASUREMENTS IDENTIFICATION SYSTEM}

Samples were identified by a five element code:

Type sample . . . . . . . . . . . . . . . . . Element 1

Component No. .................... Element 2

Component Name $\ldots \ldots \ldots \ldots \ldots \ldots \ldots$ Element 3

Sequential Number . . . . . . . . . . . . . . . . Element 4

Location $\ldots \ldots \ldots \ldots \ldots \ldots \ldots \ldots \ldots \ldots \ldots \ldots \ldots \ldots$ Element 5

Following are the notations used for each element.

\section{Type of sample}

SA Smear

LA Smear

Q-tip smear

water

scrapings

air
$100 \mathrm{~cm}^{2}$ smears

$>100 \mathrm{~cm}^{2}$ smears

Q-tips were used for very small or difficult to reach areas

sample of water from a line or component

metal surface was scrapped with a blunt instrument

Retrospective air sample 


\section{Component No. and Name}

The component numbers and names given below are shown in Figure 2.

\begin{tabular}{|c|c|c|c|c|c|}
\hline$\# 1$ & $\begin{array}{c}\text { Strainer with Monel } \\
\text { Screen }\end{array}$ & $\# 11$ & $\begin{array}{c}\text { Mixed Bed } \\
\text { Exchange Vessel }\end{array}$ & $\# 21$ & Filter \\
\hline \#2 & 50 gal Surge Tank & \#12 & Acid Regenerant Tank & $\# 22$ & Trench \\
\hline \#3 & Forwarding Pump & \#13 & Lead Grid Agitator & \#23 & Building \\
\hline \# 4 & Influent \& Effluent Filters & \#14 & Acid Foot Pump & $\# 24$ & Manhole \\
\hline \# 5 & Flow Indicator & $\# 15$ & Caustic Regenerant Tank & $\# 25$ & Pit \\
\hline \# 6 & Water Meter & \#16 & Caustic Mixer & $\# 26$ & Effluent Pipe \\
\hline \# 7 & Acid \& Caustic Ejectors & \#17 & $\begin{array}{l}\text { Conductivity } \\
\text { Indicator }\end{array}$ & $\# 27$ & $\begin{array}{c}\text { Miscellaneous items } \\
\text { in the building }\end{array}$ \\
\hline$\# 8$ & $\begin{array}{c}\text { Inlet \& Outlet } \\
\text { Pressure Gauges }\end{array}$ & \#18 & Blower & \#28 & $\begin{array}{c}\text { Exchanger Testing } \\
\text { Equipment }\end{array}$ \\
\hline \# 9 & $\begin{array}{c}\text { Inlet \& Outlet } \\
\text { Sampling Cocks }\end{array}$ & \#19 & Closed Drain Tank & $\# 29$ & Influent Pipe \\
\hline \#10 & Cation Exchange Vessel & $\# 20$ & Drain Pump & \#30 & Air Samples \\
\hline
\end{tabular}

\section{Location}

This field gives information that further specifies the part of the component that was sampled.

\section{RELEASE CRITERIA AND BACKGROUND VALUES}

To interpret the quantitative results of the characterization, the data were compared to currently accepted release criteria and background values typically found in the natural environment.

Following are the principal release and background values:

Surface Contamination Release Criteria - Removable and total surface contamination limits (for unrestricted release of nonporous materials) used by the USNRC and by DOE are given in USNRC Regulatory Guide 1.86 (reference 1) and are listed in the following Table 1. The Regulatory Guide 1.86 values were adopted by DOE and are also listed in DOE Order 5400.5A (reference 2) and the draft rule 10 CFR 834 (reference 3 ).

Lead in paint release criteria - paint is considered to be lead-containing if it has greater than $0.5 \%$ lead by weight. 


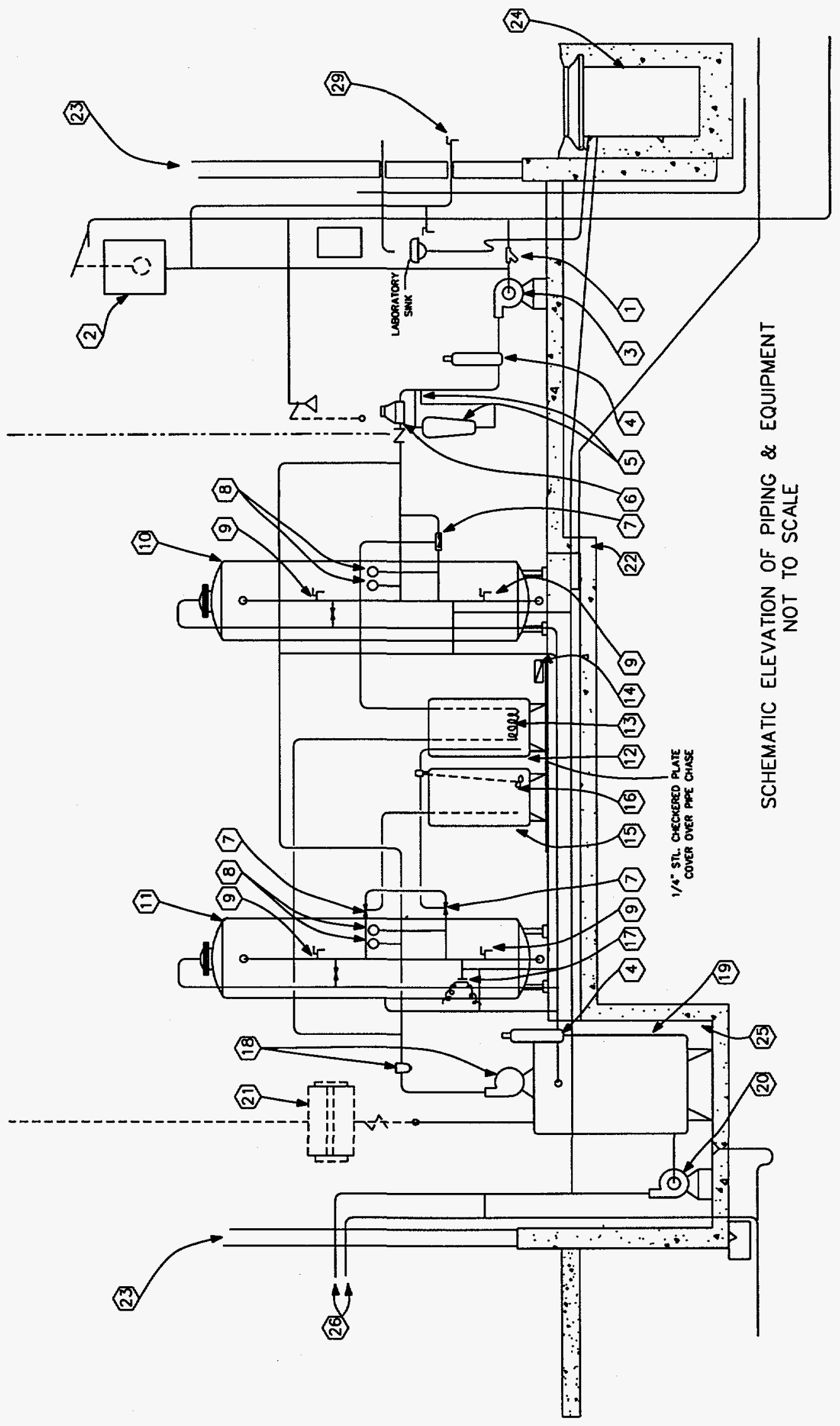

葛 
TABLE 1. Allowable Residual Surface Contamination Limits $\left(\mathrm{dpm} / 100 \mathrm{~cm}^{2}\right)$

\begin{tabular}{lrrr}
\hline \multicolumn{1}{c}{ Total (fixed and removable) Radionuclides } & Average & Maximum & Removable \\
\hline $\begin{array}{l}\text { Transuranics, I-125, I-129, Ra-226, Ac-227, } \\
\text { Ra-228, Th-228, Th-230, Pa-231. }\end{array}$ & 100 & 300 & 20 \\
$\begin{array}{l}\text { Th-Natural, Sr-90, I-126, I-131, I-133, Ra-233, } \\
\text { Ra-224, U-232, Th-232. }\end{array}$ & 1,000 & 3,000 & 200 \\
$\begin{array}{l}\text { U-Natural, U-235, U-238, and associated decay } \\
\text { product, alpha emitters }\end{array}$ & 5,000 & 15,000 & 1,000 \\
\begin{tabular}{l} 
Beta-gamma emitters (except Sr-90) \\
\hline
\end{tabular} & 5,000 & 15,000 & 1,000 \\
\hline
\end{tabular}

Background radiation exposure rate - the radiation exposure rate inside most buildings at ANL-E is typically $10-15 \mu \mathrm{R} / \mathrm{h}$. This value is consistent with the natural background exposure rate in the midwestern USA (reference 4). The environmental penetrating radiation from areas surrounding ANL were $92 \pm 4 \mathrm{mrem} / \mathrm{y}(10.5 \pm 0.5 \mu \mathrm{R} / \mathrm{h})$; from the ANL boundaries the penetrating radiation ranged from $89 \pm 6 \mathrm{mrem} / \mathrm{y}(10.2 \pm 0.7 \mu \mathrm{R} / \mathrm{h})$ to $129 \pm 12 \mathrm{mrem} / \mathrm{y}(14.7 \pm 1.4 \mu \mathrm{R} / \mathrm{h})($ from reference 8).

Background radioactivity in soil - the activity concentration in soil is predominantly due to naturally occurring radionuclides in the uranium and thorium series. Some non natural radionuclides found in soil are due to atmospheric fallout. Typical concentrations in soil of the major natural and fallout radionuclides are listed in Table 2 from reference 8 .

Asbestos release criteria - a material (e.g., insulation, pipe joint compound, floor tile) is considered asbestos-containing if it contains greater than $1 \%$ asbestos by weight.

Hazardous material release criteria - a RCRA (Resource Conservation Recovery Act) hazardous waste is defined as waste which meets one of the following two criteria:

1. it exhibits the 40 CFR 261.20 (reference 7) specific properties of

- ignitability

- corrosivity

- reactivity

- toxicity, and

2. it is listed in 40 CFR 265 (reference 7) as a RCRA hazardous waste, and it exceeds the specified concentration limits. 
TABLE 2. Typical Background Concentrations in Soil and Sediments

\begin{tabular}{|c|c|c|c|c|}
\hline \multirow[b]{2}{*}{ Nuclide } & \multicolumn{2}{|c|}{ Soil Average } & \multicolumn{2}{|c|}{ Bottom Sediment Average } \\
\hline & ANL Perimeter & Off-Site & ANL Perimeter & Off-Site \\
\hline & \multicolumn{4}{|c|}{ Activity $\mathrm{pCi} / \mathrm{g}$} \\
\hline${ }^{40} \mathrm{~K}$ & $18.48 \pm 5.42$ & $16.55 \pm 5.79$ & $12.23 \pm 10.92$ & $14.50 \pm 14.37$ \\
\hline${ }^{137} \mathrm{Cs}$ & $0.62 \pm 0.37$ & $0.53 \pm 0.90$ & $0.86 \pm 2.15$ & $0.14 \pm 0.55$ \\
\hline${ }^{226} \mathrm{Ra}$ & $1.02 \pm 0.41$ & $1.04 \pm 0.51$ & $0.73 \pm 0.56$ & $0.99 \pm 0.94$ \\
\hline${ }^{228} \mathrm{Th}$ & $1.08 \pm 0.41$ & $1.05 \pm 0.71$ & $0.62 \pm 0.67$ & $1.15 \pm 2.37$ \\
\hline \multirow[t]{2}{*}{${ }^{232} \mathrm{Th}$} & $0.81 \pm 0.25$ & $0.83 \pm 0.62$ & $0.50 \pm 0.48$ & $0.94 \pm 2.19$ \\
\hline & \multicolumn{4}{|c|}{ Activity fCi/g } \\
\hline${ }^{238} \mathrm{Pu}$ & $0.9 \pm 0.3$ & $0.5 \pm 0.3$ & $2.2 \pm 6.8$ & $0.3 \pm 1.3$ \\
\hline${ }^{239} \mathrm{Pu}$ & $15.0 \pm 2.1$ & $11.2 \pm 4.9$ & $159.4 \pm 721.7$ & $6.2 \pm 25.5$ \\
\hline${ }^{241} \mathrm{Am}$ & $5.3 \pm 0.9$ & $4.4 \pm 1.8$ & $16.8 \pm 58.5$ & $2.4 \pm 7.8$ \\
\hline
\end{tabular}

Airborne radioactivity limits - annual average concentration guidelines for the workplace are listed as derived air concentrations (DAC) in the DOE rule 10 CFR 835 (reference 5). However, the concentration of non naturally occurring radionuclides is expected to be non detectable in non radiological areas.

Retention tanks radioactivity release limits - at ANL-E, laboratory waste water is collected in retention tanks and is sampled and analyzed for alpha and beta radioactivity before being released to the laboratory waste water treatment plant. The ANL site release limits are $0.03 \mathrm{pCi} / \mathrm{ml}(0.067$ $\mathrm{dpm} / \mathrm{ml}$, the derived concentration guide for $\left.{ }^{239} \mathrm{Pu}\right)$ for alpha activity, and $1.0 \mathrm{pCi} / \mathrm{ml}(2.22 \mathrm{dpm} / \mathrm{ml}$, the derived concentration guide for ${ }^{90} \mathrm{Sr}$ ) for beta activity. Liquid waste in retention tanks exceeding ten times these limits is processed to remove the contamination, normally by evaporation, and the residue disposed of as radioactive waste. 


\section{INSTRUMENTATION}

Instruments were chosen to assure that the MDAs for the selected instrument/technique was less than the release criteria. A listing of the principal instruments used and their typical MDAs is given in Table 3. Photographs of the principal instruments used for the characterization are shown in Figures 3 through 7 .

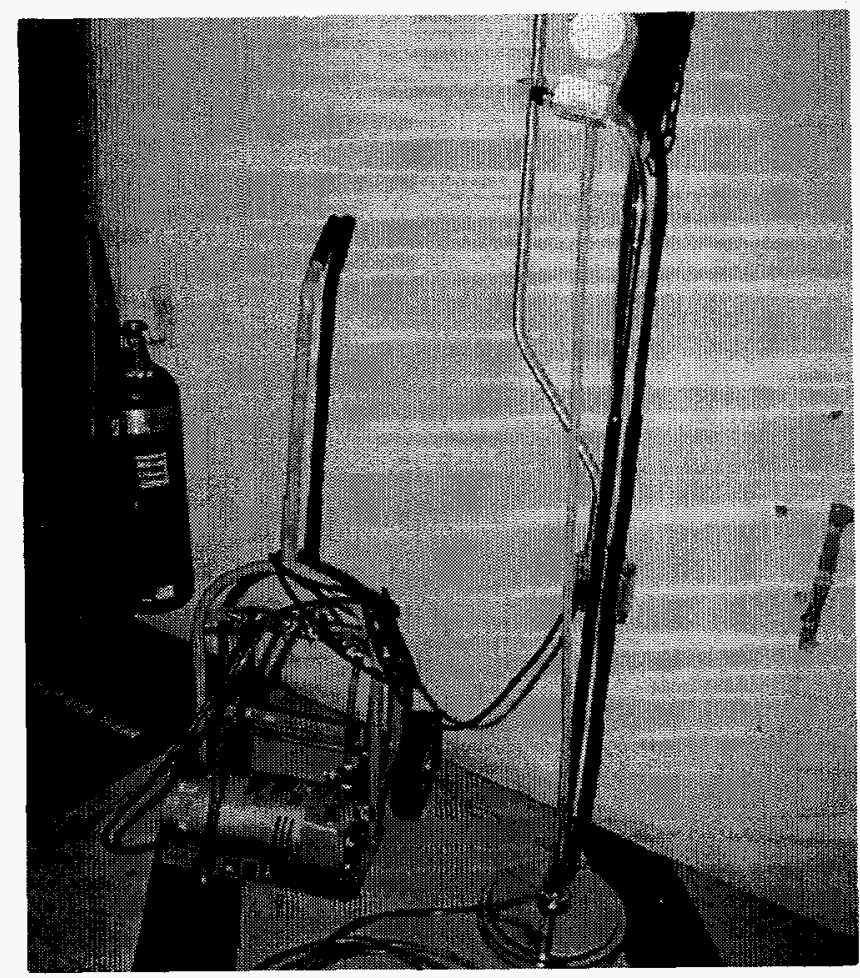

FIGURE 3. Retrospective Air Sampling System (Giraffe) 
TABLE 3. Instruments Used for Characterization

\begin{tabular}{|c|c|c|c|c|c|}
\hline Purpose & Instrument & $\begin{array}{c}\text { Detector } \\
\text { Description }\end{array}$ & $\begin{array}{l}\text { Measurement } \\
\text { Units }\end{array}$ & Typical Characteristics & $\begin{array}{l}\text { Typical } \\
\text { MDA }^{\mathrm{a}}\end{array}$ \\
\hline Total Surface $(\alpha)$ & $\begin{array}{l}\text { NE Technology, } \\
\text { Ltd. ELECTRA }\end{array}$ & $\begin{array}{c}\text { Dual Scintillator } \\
100 \mathrm{~cm}^{2} \text { sampling area } \\
0.5 \mathrm{mg} / \mathrm{cm}^{2}\end{array}$ & $\mathrm{cpm}$ & $\begin{array}{r}21 \%{ }^{241} \text { Am efficiency, } \\
30 \text { sec residence time, } \\
\leq 7 \mathrm{cpm} \text { background }\end{array}$ & $98 \mathrm{dpm}$ \\
\hline Total Surface $(\beta-\gamma)$ & $\begin{array}{l}\text { NE Technology, } \\
\text { Lid. ELECTRA }\end{array}$ & $\begin{array}{c}\text { Dual Scintillator } \\
100 \mathrm{~cm}^{2} \text { sampling area } \\
\sim 6 \mathrm{mg} / \mathrm{cm}^{2}\end{array}$ & $\mathrm{cpm}$ & $\begin{array}{l}29 \%{ }^{90} \mathrm{Sr}-\mathrm{Y} \text { efficiency, } \\
30 \mathrm{sec} \text { residence time, } \\
\leq 400 \mathrm{cpm} \text { background }\end{array}$ & $410 \mathrm{dpm}$ \\
\hline Removable Surface $(\alpha)$ & Tennelec APC & $\begin{array}{c}\text { Gas Proportional } \\
5 \mathrm{~cm} \mathrm{dia.} \\
0.1 \mathrm{mg} / \mathrm{cm}^{2}\end{array}$ & $\mathrm{cpm}$ & $\begin{array}{l}30 \%{ }^{241} \mathrm{Am} \text { efficiency, } \\
2 \mathrm{~min} \text { count time } \\
\leq 1 \mathrm{cpm} \text { background }\end{array}$ & $17 \mathrm{dpm}$ \\
\hline Removable Surface $(\beta-\gamma)$ & Tennelec APC & $\begin{array}{c}\text { Gas Proportional } \\
5 \mathrm{~cm} \mathrm{dia} . \\
0.1 \mathrm{mg} / \mathrm{cm}^{2} \\
\end{array}$ & cpm & $\begin{array}{l}42 \%{ }^{90} \mathrm{Sr}-\mathrm{Y} \text { efficiency, } \\
2 \text { min count time, } \\
\leq 40 \mathrm{cpm} \text { background }\end{array}$ & $86 \mathrm{dpm}$ \\
\hline Removable Surface $(\alpha)$ & DABRAS & $\begin{array}{l}\text { Gas Proportional } \\
200 \mathrm{~cm}^{2} \\
0.4 \mathrm{mg} / \mathrm{cm}^{2} \\
\end{array}$ & $\mathrm{cpm}$ & $\begin{array}{l}27 \%{ }^{241} \text { Am efficiency, } \\
2 \text { min count time, } \\
\leq 2 \mathrm{cpm} \text { background }\end{array}$ & $18 \mathrm{dpm}$ \\
\hline Removable Surface $(\beta-\gamma)$ & DABRAS & $\begin{array}{l}\text { Gas Proportional } \\
200 \mathrm{~cm}^{2} \\
0.4 \mathrm{mg} / \mathrm{cm}^{2} \\
\end{array}$ & cpm & $\begin{array}{l}49 \%{ }^{90} \mathrm{Sr}-\mathrm{Y} \text { efficiency, } \\
2 \text { min count time, } \\
\leq 270 \mathrm{cpm} \text { background }\end{array}$ & $86 \mathrm{dpm}$ \\
\hline Find Elevated $x-\gamma$ & $\begin{array}{c}\text { Eberline } \\
\text { PRM 5-3 with } \\
\text { PG-2 detector }\end{array}$ & $\begin{array}{c}5 \mathrm{~cm} \text { diameter } \times 2 \mathrm{~mm} \\
\text { thick } \mathrm{NaI}(\mathrm{Tl})\end{array}$ & cpm & $\begin{array}{l}2 \mathrm{sec} \text { residence time } \\
500 \mathrm{cpm} \text { background }\end{array}$ & $1500 \mathrm{cpm}$ net \\
\hline
\end{tabular}

a Note: The typical MDA for the NE Technology, Ltd. ELECTRA, the Ludlum Model 3 and the Eberline PRM 5-3 with a PG-2 detector is based upon the use of audio output to enhance the discernment of recordable measurements. 


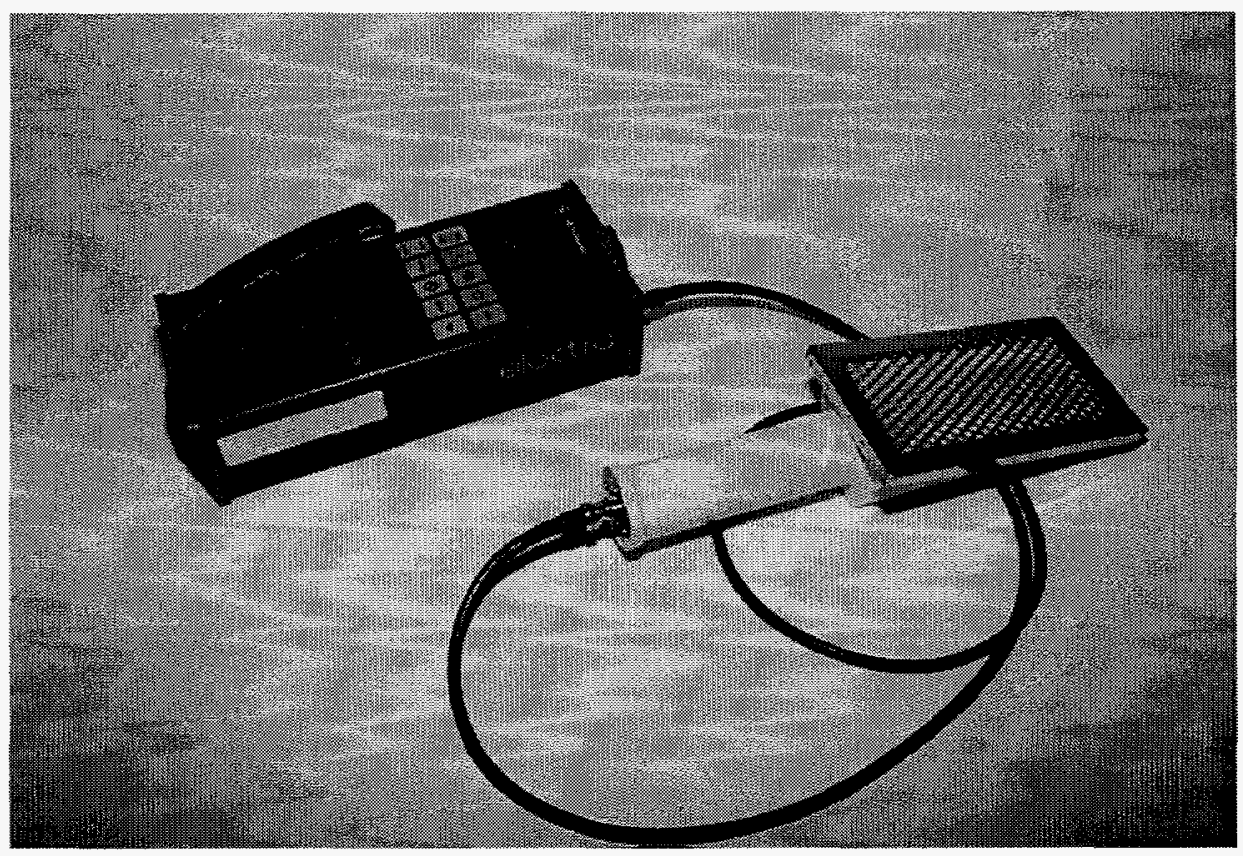

FIGURE 4. NE Technology Model Electra with $100 \mathrm{~cm}^{2}$ Detector for Measuring $\alpha \beta \gamma$ Surface Contamination

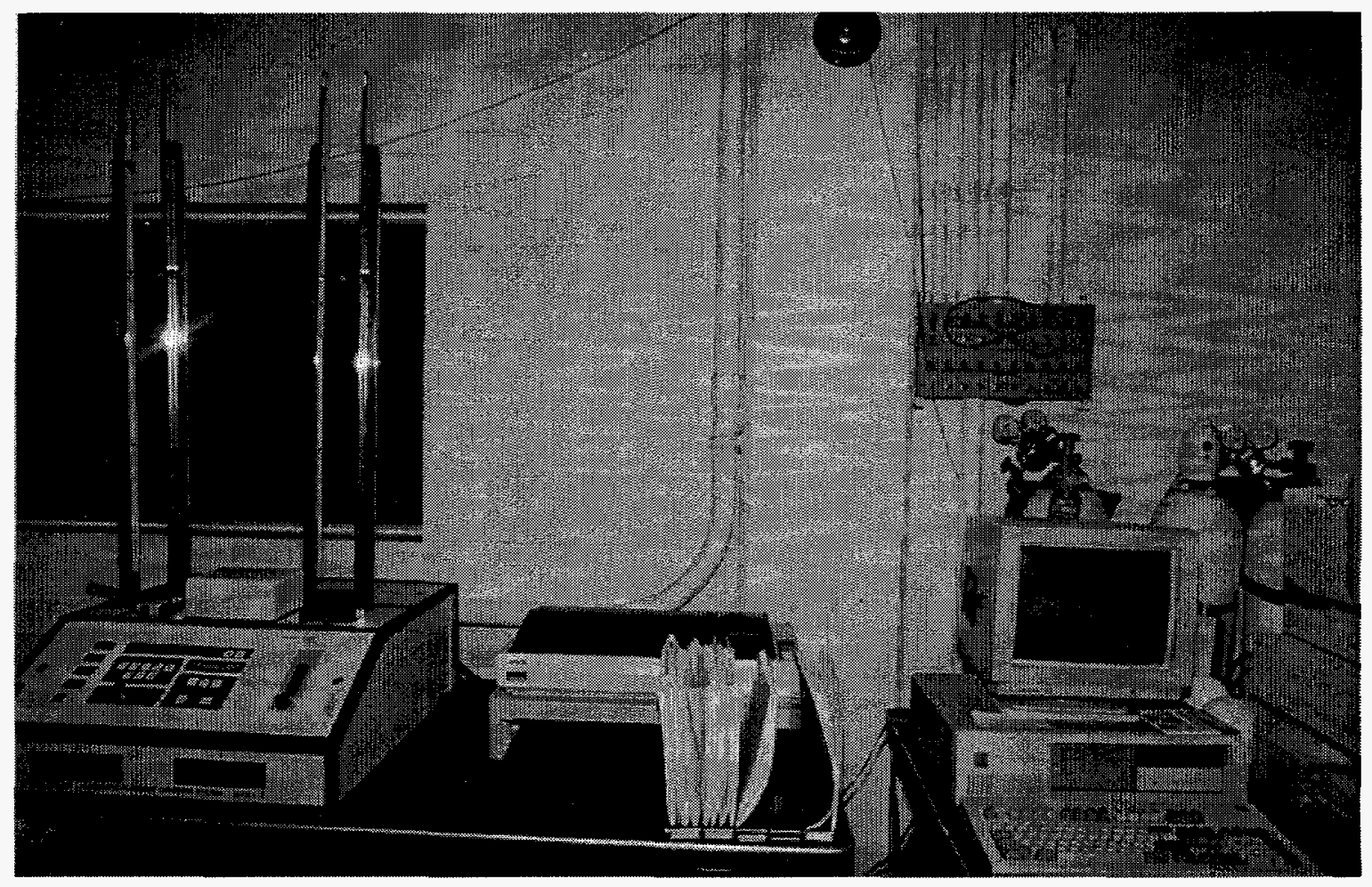

FIGURE 5. Tennelec APC MII Smear Counting System 


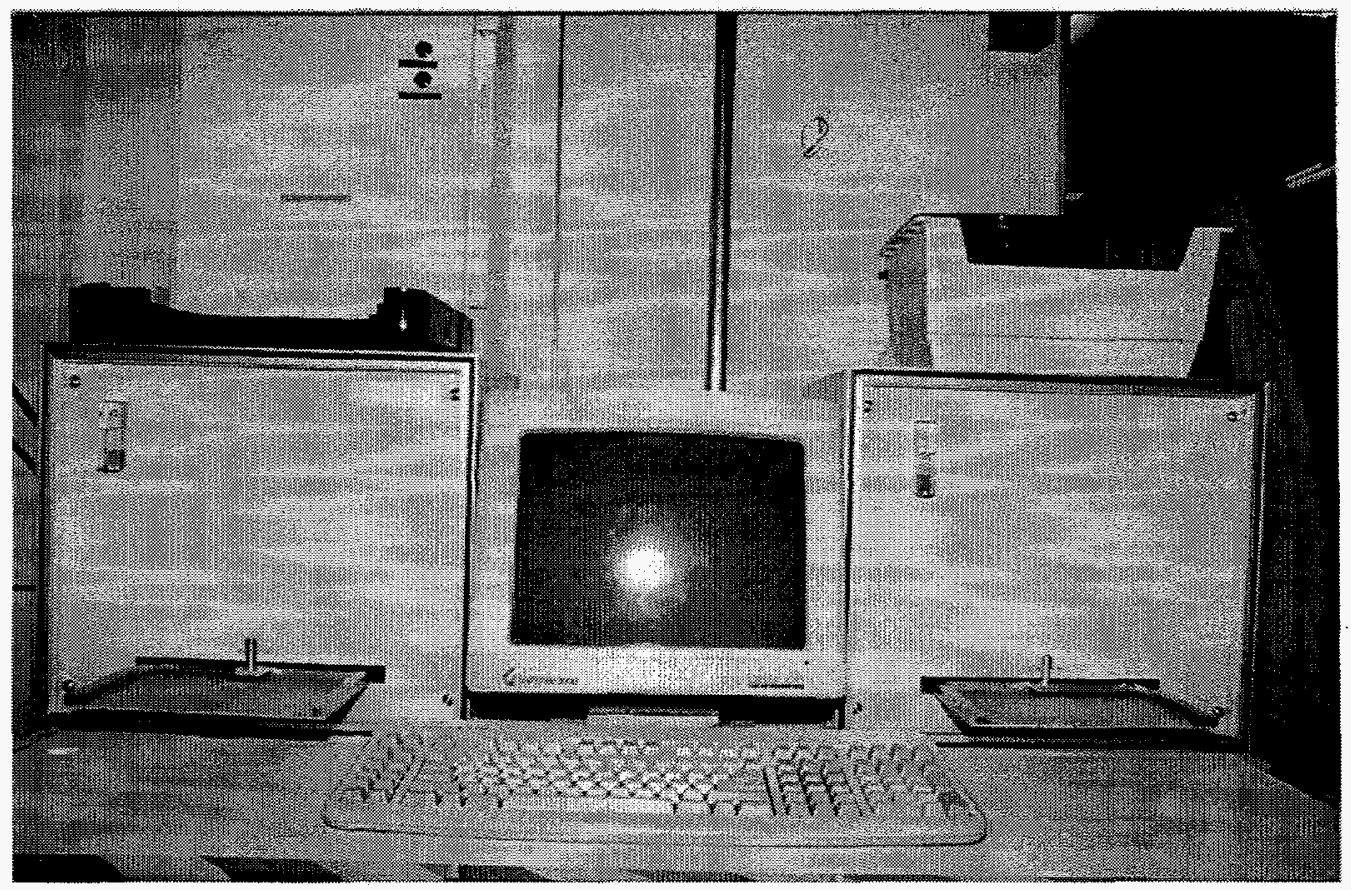

FIGURE 6. Dual Alpha Beta Radioactivity Assay System (DABRAS) for smear counting

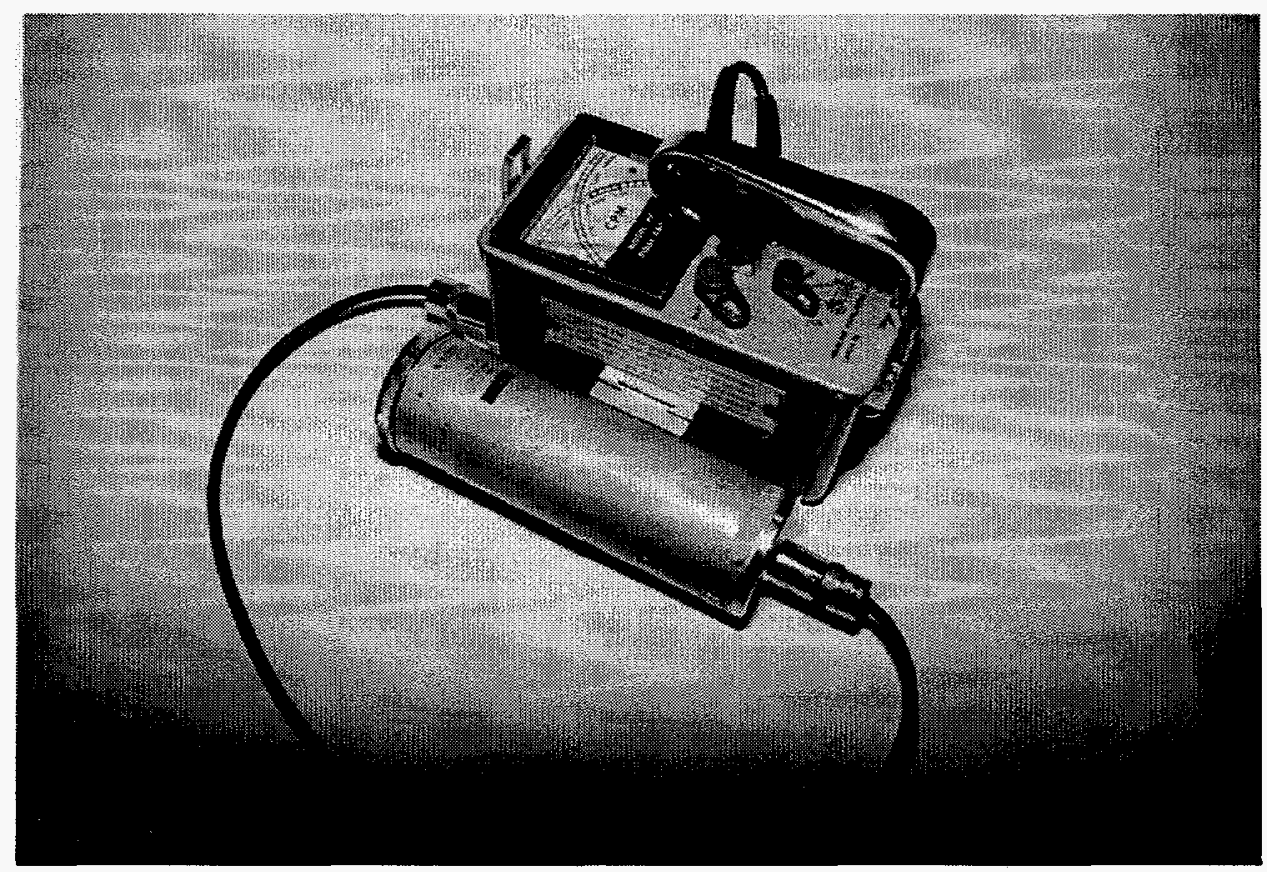

FIGURE 7. Eberline PRM-5-3 with PG-2 Scintillation Detector $\left(2^{\prime \prime} \times 2 \mathrm{~mm}\right.$ thick $\mathrm{NaI}$ detector) 


\section{NUMBER OF MEASUREMENTS AND SAMPLES}

A listing of the type and number of measurements performed and samples collected during the characterization is given in Table 4 .

\section{RESULTS}

\section{A. Surprises}

The characterization identified the following unexpected conditions:

1. There was water in the lines

TABLE 4. Measurements \& Samples Required for Retention Tank Characterization

\begin{tabular}{lr}
\hline \multicolumn{1}{c}{ Item } & Total Number \\
\hline & \\
Type of measurement & 23 \\
General $\alpha$ and $\beta$ scan & 23 \\
General exposure rate & \\
& \\
Type of samples collected & 50 \\
Smears & 1 \\
Scrapings & 1 \\
Asbestos & 11 \\
Water and Resin & 2 \\
Air & \\
& \\
Type of sample analyses & 64 \\
Gross $\alpha \beta \gamma$ & 3 \\
$\gamma$ spectroscopy & 1 \\
Asbestos & 6 \\
Hazardous materials & 3 \\
pH & 11 \\
Metals & \\
\hline
\end{tabular}


2. There was water in the Cation and Mixed Bed Exchange Vessels

3. There was water in the Manhole

4. A pipe in the trench connected to the Building 575 Manhole

5. A pipe from the pit went under the floor and drained to a lagoon

6. A pipe from the trench went under the concrete under the exchange vessels.

\section{B. Lead in Paint Results}

The pipes and tanks are not painted. Therefore there were no tests for lead in paint.

\section{Asbestos Results}

The pipe in the trench has insulation covering it. This was tested for asbestos. None was found. The insulation is concrete.

\section{Air Sample Results}

Two grab air samples were collected during the sampling period. There were low levels of long lived activity on the samples; up to $4.7 \pm 3.0 \mathrm{dis} / \mathrm{min} \alpha$ and $37 \pm 10 \mathrm{dis} / \mathrm{min} \beta$ on the filters. These are assumed to be naturally occurring radionuclides.

\section{E. Vessels and Pipes}

The main components of the waste Ion Exchange Facility are the exchange vessels and the associated piping. The original plans were to open the lines and take smears at strategic locations to assess the internal contamination. However, there is an aqueous solution in much of the piping, in the cation and mixed bed exchange vessels. Instead of smears, water samples were removed from the five locations indicated in Table 5. The samples were counted for gross $\alpha$ and $\beta$ activities in the Control Laboratory (ESH-DA). The water from the influent filter (\#4), the Cation Exchange Vessel (\#10) and the Mixed Bed Exchange Vessel (\#11) had no activity in the water above the retention tank release criteria. A more detailed summary of the water samples is given in Appendix C. 
TABLE 5. Gross $\alpha$ and $\beta$ Activity in Water Samples

\begin{tabular}{|c|c|c|c|c|c|}
\hline \multirow[b]{2}{*}{ Date } & \multirow[b]{2}{*}{ Location } & \multirow{2}{*}{$\begin{array}{l}\text { Sample } \\
\text { Number }\end{array}$} & \multirow{2}{*}{$\begin{array}{l}\text { Request } \\
\text { Number }\end{array}$} & \multicolumn{2}{|c|}{$\mathrm{dpm} / \mathrm{mL}$} \\
\hline & & & & $\alpha$ & $\beta$ \\
\hline $9 / 18 / 96$ & \#4 Influent Filter & 45 & 6469 & 0.069 & 0.218 \\
\hline $9 / 18 / 96$ & \#10 lower drain & 46 & 6470 & 0.040 & 0.324 \\
\hline $9 / 18 / 96$ & \#11 lower drain & 47 & 6471 & 0.044 & 0.871 \\
\hline $9 / 18 / 96$ & \#11 upper drain & 48 & 6472 & 0.039 & 0.486 \\
\hline $9 / 18 / 96$ & \#24 outside & 50 & 6474 & 0.03 & 0.10 \\
\hline $9 / 24 / 96$ & $\# 10$ tank & 57 & 6417 & 0.03 & 0.004 \\
\hline \multirow[t]{2}{*}{$9 / 25 / 96$} & \#24 inside & 67 & 6414 & 0.37 & 0.99 \\
\hline & Site Release Criteria ${ }^{a}$ & & & 0.067 & 2.22 \\
\hline
\end{tabular}

a The Department of Energy Derived Concentration Guide (DCG) limits

Some of the dry smears were counted in the DABRAS and some were counted on the Tennelec (see Table 3). For ease of evaluation, Table B1 in Appendix B combines the data from both counters; Table B2 has the data from the Tennelec; and Table B3 has the data from the DABRAS. Several components had elevated activities; \#10 the cation exchange vessel, $28 \pm 15 \mathrm{dis} / \mathrm{min} \alpha$ and $105 \pm 28$ $\mathrm{dis} / \mathrm{min} \beta ; \# 12$ the acid regenerant tank, $172 \pm 52 \mathrm{dis} / \mathrm{min} \beta ; \# 13$ the mixer in the acid tank, $84 \pm 49$ $\mathrm{dis} / \mathrm{min} \beta$; \#19 the closed drain tank, $106 \pm 29 \mathrm{dis} / \mathrm{min} \alpha$ and $48 \pm 22 \mathrm{dis} / \mathrm{min} \beta$; and \#15 the caustic regenerant tank, $58 \pm 48 \mathrm{dis} / \mathrm{min} \beta$. No isotopic identification was made of the activities on the smears.

Two water samples and a resin sample from the exchange vessels and the closed drain tank were sent to ACL for hazardous constituent analysis. The water sample from the closed drain tank (\#19) had no detectable contaminants. The water sample from the mixed bed exchange vessel (\#11) had no metal or radioactive contaminants. However, there was a low concentration of the semivolatile organic compound bis(2-ethylhexyl) phthalate, below the toxicity characteristic level. The resin sample from the cation exchange vessel (\#10) contains levels of semivolatile organic compounds less than the toxicity characteristic level; radionuclides up to 1420 times the background levels for ${ }^{241} \mathrm{Am}$; and metal contaminants. The only organic compound that was not found in the blank was bis(2-ethylhexyl) phthalate. The metal contaminants could be up to 11 times the toxicity characteristic level chromium. This level of contaminants indicates the need for a TCLP analysis that measures the amount of metals that will actually be leached from the resin. This analysis was requested on February 6, 1997. The only metal concentrations in the resin leachate above the 
detection level are mercury and barium. Because their concentrations are below the regulatory level, the resin is a radioactive waste not a mixed waste. Both the cation exchange resin sample and the water sample from the closed drain tank are acidic with a pH between 2 and 3 . The results are summarized in Table 6 and presented in detail in Table D1 followed by the supporting documentation in Appendix D.

\section{F. Manhole and Subsurface Piping}

The piping from the facility is connected to other facilities (see Figure 1) such as building 575 and a lagoon. The other end of some of the piping appears to end in the laboratory tanks and equalization tanks. One water sample, \#67, from the manhole (component \#24) had activity above the release criteria for the site (see Table 5). Since it is less than ten times the site release criteria, it does not need any processing before it is released to the on site drains. A smear sample, \#44, from the top sides had elevated activity $87 \pm 49 \mathrm{dis} / \mathrm{min} \beta$. The scraping sample, $\# 55$, appeared to be naturally occurring radioactivity $\left({ }^{238} \mathrm{U}\right.$ and ${ }^{232} \mathrm{Th}$ daughters).

TABLE 6. Cation Exchange Resin Sample dated 9/20/96

\begin{tabular}{lll}
\hline \multicolumn{1}{c}{ Analysis } & \multicolumn{1}{c}{$\begin{array}{c}\text { Highest } \\
\text { Concentration/Activity }\end{array}$} & $\begin{array}{c}\text { Limit or } \\
\text { Background Values }\end{array}$ \\
\hline $\mathrm{pH}$ & 2.4 most acidic & $\leq 2 \mathrm{or} \geq 12.5$ \\
Volatile Organic & none detected & $>$ detection level ${ }^{\mathrm{a}}$ \\
Semivolatile Organic & $912 \mu \mathrm{g} / \mathrm{L}$ & $1,000,000 \mu \mathrm{g} / \mathrm{L}$ \\
bis(2-Ethylhexyl) Phthalate & & \\
Mercury Metal & $0.49 \mu \mathrm{g} / \mathrm{L}$ & $200 \mu \mathrm{g} / \mathrm{L}$ \\
Barium Metal & $0.20 \mu \mathrm{g} / \mathrm{mL}$ & $100 \mu \mathrm{g} / \mathrm{mL}$ \\
${ }^{241}$ Am radionuclide & $24.1 \mathrm{pCi} / \mathrm{g}$ & $0.017 \mathrm{pCi} / \mathrm{g}$ \\
${ }^{137} \mathrm{Cs}$ radionuclide & $2.6 \mathrm{pCi} / \mathrm{g}$ & $0.86 \mathrm{pCi} / \mathrm{g}$ \\
${ }^{60}$ Co radionuclide & $4.3 \mathrm{pCi} / \mathrm{g}$ & $\mathrm{BDL}^{\mathrm{b}}$ \\
\hline
\end{tabular}

a Currently list of compounds regulated under RCRA has individual limits above the detection level.

b $\mathrm{BDL}=$ Below detectable levels. 


\section{G. Building Interior, Trench and Pit}

No elevated activity was found on the smears of the trench. Low levels of $\beta$ activity were found on the floor $58 \pm 48 \mathrm{dis} / \mathrm{min}$ and the west wall $76 \pm 48 \mathrm{dis} / \mathrm{min}$ of building 579 . No elevated activity was found on the smears of the pit. There are many miscellaneous items stored or abandoned in the building. Smears of these items showed a maximum of $53 \pm 49 \mathrm{dis} / \mathrm{min} \beta$ on a mixer on the table. This mixer may not be related to the Waste Ion Exchange Facility.

Direct surveys of the Building detected no alpha or beta activity above background with the NE electra. No gamma activity above background was detected with the PG-2 detector.

\section{SUMMARY OF RESULTS ABOVE BACKGROUND}

Several locations, the floor and west wall of building 579 and the manhole, had low levels of $\beta$ activity, up to $87 \pm 49 \mathrm{dis} / \mathrm{min}$. These values are below the allowable residual surface contamination limits for removable beta activity.

The internal surfaces of the pipes, tanks and columns had higher levels of $\beta$ activity up to $172 \pm 52$ $\mathrm{dis} / \mathrm{min}$ and some $\alpha$ activity up to $106 \pm 29 \mathrm{dis} / \mathrm{min}$. The beta values are below the allowable residual removable surface contamination limits. Depending upon the isotopes involved the alpha values are above the allowable residual removable surface contamination limits for transuranics such as ${ }^{241} \mathrm{Am}$.

The resin sample had elevated concentrations of RCRA metals, up to 11 times the toxicity characteristic level for chromium. The leachate from the resin had no metal concentrations above the toxicity characteristic level. The resin sample also had radionuclide concentrations up to 1,420 times the background level for ${ }^{241} \mathrm{Am}$ and detectable ${ }^{60} \mathrm{Co}$ levels. ${ }^{60} \mathrm{Co}$ concentrations in the background samples are below the instrument detection levels.

Two of the samples were acidic, with $\mathrm{pH}$ values between 2 and 3 .

\section{ESTIMATE OF INVENTORY OF ACTIVITY IN COLUMNS}

\section{A. Water in Facility}

Water was found in four components and resin was found in one. Volume estimates were calculated for the water and resin and are listed in Table 7 with the calculational parameters. 
Table 7. Volumes in the Vessels, Tanks \& Manhole

\begin{tabular}{|c|c|c|c|c|}
\hline Dimensions & $\begin{array}{l}\text { Mixed Bed } \\
\text { Exchange } \\
\text { Vessel }\end{array}$ & $\begin{array}{c}\text { Cation } \\
\text { Exchange } \\
\text { Vessel }\end{array}$ & $\begin{array}{l}\text { Closed } \\
\text { Drain Tank }\end{array}$ & Manhole \\
\hline Vessel height $(\mathrm{ft})=\mathrm{h}$ & 8.5 & 8.5 & 4 & 3.83 \\
\hline circumference $(\mathrm{ft})=2 \pi \mathrm{r}$ & 7.92 & 7.92 & 9.42 & 6.28 \\
\hline radius $(\mathrm{ft})=\mathrm{r}$ & 1.25 & 1.25 & 1.5 & 1 \\
\hline Vessel Volume $\left(\mathrm{ft}^{3}\right) \mathrm{V}=\pi \mathrm{r}^{2} \mathrm{~h}$ & 41.7 & 41.7 & 28.3 & 12.0 \\
\hline Vessel Volume $(\mathrm{L})=30.48^{3} \mathrm{~V} / 1000$ & 1,180 & 1,180 & 801 & 341 \\
\hline Vessel Volume $(\mathrm{gal})=7.841 \mathrm{~V}$ & 312 & 312 & 212 & 90.1 \\
\hline resin height $(\mathrm{ft})=\mathrm{h}_{\mathrm{r}}$ & & 5.83 & & \\
\hline Resin Volume $\left(\mathrm{ft}^{3}\right) \mathrm{V}_{\mathrm{r}}=0.1965 \pi \mathrm{r}^{2} \mathrm{~h}_{\mathrm{r}}$ & & 5.63 & & \\
\hline Resin Volume $(\mathrm{L})=30.48^{3} \mathrm{~V}_{\mathrm{r}} / 1000$ & & 159 & & \\
\hline Resin Volume $(\mathrm{gal})=7.841 \mathrm{~V}_{\mathrm{r}}$ & & 42.1 & & \\
\hline water level height $(\mathrm{ft})=\mathrm{h}_{\mathrm{w}}$ & 5.67 & 7.5 & 1.25 & 2.67 \\
\hline $\begin{array}{l}\text { Water Volume }\left(\mathrm{ft}^{3}\right) \\
\mathrm{V}_{\mathrm{w}}=0.2 \pi \mathrm{r}^{2} \mathrm{~h}_{\mathrm{r}}+\pi \mathrm{r}^{2}\left(\mathrm{~h}_{\mathrm{w}}-\mathrm{h}_{\mathrm{r}}\right)\end{array}$ & 27.8 & 31.2 & 8.84 & 8.38 \\
\hline Water Volume $(\mathrm{L})=30.48^{3} \mathrm{~V}_{\mathrm{w}} / 1000$ & 788 & 833 & 250 & 237 \\
\hline Water Volume $(\mathrm{gal})=7.841 \mathrm{~V}_{\mathrm{w}}$ & 208 & 233 & 66.1 & 62.7 \\
\hline
\end{tabular}

Two of the components in Table 7 have concentrations of contaminants that are of concern. An assumed resin density of $0.95 \mathrm{~g} / \mathrm{ml}$ gives a total resin mass of $151 \mathrm{~kg}$. The resin sample was $19.65 \%$ solids by weight. All of the contaminants in the cation exchange vessel are assumed to be associated with the resin. The water in the cation exchange vessel is assumed to have only a low level of semivolatile organic compounds similar to those found in the mixed bed exchange vessel and the closed drain tank. The total calculated amount of RCRA metal contaminants on the resin are given in Table 8. The total calculated amount of radioactive contaminants on the resin are given in Table 9.

\section{B. Surface Contamination in the Facility}

There are two small area smears $\left(100 \mathrm{~cm}^{2}\right)$ that had alpha activity above the release criteria for removable surface contamination found in Table 1 for transuranic nuclides. One was from inside the Cation Exchange Vessel. If that smear was representative of the activity on the entire $9,190 \mathrm{~cm}^{2}$ inside surface, then there are $1,160 \mathrm{pCi}$ of alpha activity inside the vessel. The second smear was 
TABLE 8. RCRA Metal Contamination in the Cation Exchange Vessel

\begin{tabular}{lcr}
\hline Contaminant & $\begin{array}{c}\text { Concentration } \\
(\mathrm{mg} / \mathrm{kg})\end{array}$ & $\mathrm{mg}$ \\
\hline $\mathrm{Cd}$ & 20.7 & 3,130 \\
$\mathrm{Cr}$ & 1120 & 169,500 \\
$\mathrm{~Pb}$ & 248 & 37,500 \\
$\mathrm{Hg}$ & 8.3 & 1,250 \\
$\mathrm{Se}$ & 132 & 20,000 \\
$\mathrm{Ag}$ & 124 & 18,800 \\
\hline
\end{tabular}

TABLE 9. Radioactive Contamination in the Cation Exchange Vessel \& Manhole

\begin{tabular}{lccccc}
\hline & \multicolumn{2}{c}{ Cation Exchange Vessel } & & \multicolumn{2}{c}{ Manhole } \\
\cline { 2 - 3 } Contaminant & $\begin{array}{c}\text { Concentration } \\
(\mathrm{pCi} / \mathrm{g})\end{array}$ & $\begin{array}{c}\text { Activity } \\
(\mu \mathrm{Ci})\end{array}$ & & $\begin{array}{c}\text { Concentration } \\
(\mathrm{pCi} / \mathrm{ml})\end{array}$ & $\begin{array}{c}\text { Activity } \\
(\mu \mathrm{Ci})\end{array}$ \\
\hline${ }^{60} \mathrm{Co}$ & 4.3 & 0.65 & & & \\
${ }^{137} \mathrm{Cs}$ & 2.6 & 0.39 & & & \\
${ }^{241} \mathrm{Am}$ & 24.1 & 3.65 & & & 0.19 \\
${ }_{\mathrm{Gross}} \alpha$ & 19.2 & 2.91 & & 0.8214 & 0.52 \\
Gross $\beta$ & 4.3 & 0.65 & & 2.1978 & \\
\hline
\end{tabular}

from inside the Closed Drain Tank. If that smear was representative of the activity on the entire $13,130 \mathrm{~cm}^{2}$ inside surface, then there are $6,270 \mathrm{pCi}$ of alpha activity inside the tank.

After the water is removed from the vessels, tanks and lines, they should be surveyed to determine whether the areas accessible for smear surveys are representative of the general inside contamination levels. 


\section{LESSONS LEARNED}

Trace all pipes to and from the building.

\section{REFERENCES}

1. USNRC Regulatory Guide 1.86, "Termination of Operating Licenses for Nuclear Reactors" 1974

2. DOE Order 5400.5, "Radiation Protection of the Public and the Environment" (1990)

3. Draft DOE Rule 10 CFR 834, "Radiation Protection of the Public" (1995)

4. NCRP Report No. 94, "Exposure of the Population in the United States and Canada from Natural Background Radiation," National Council on Radiation Protection and Measurements, Bethesda, Maryland (1987)

5. DOE 10 CFR 835, "Occupational Radiation Protection" (1993)

6. EPA-5201/1-88-020, "Limiting Values of Radionuclide Intake And Air Concentration and Dose Conversion Factors For Inhalation, Submersion, And ingestion", Federal Guidance Report No. 11

7. 40 CFR Parts 260-299, Code of Federal Regulations, Protection of Environment (July 1, 1995), U.S. Government Printing Office, Washington

8. "Argonne National Laboratory-East, Site Environmental Report for Calendar Year 1995" by N. W. Golchert and R. G. Kolzow, ANL-96/3, September 1996 (UC-607) 
APPENDIX A: Documentation of Samples 


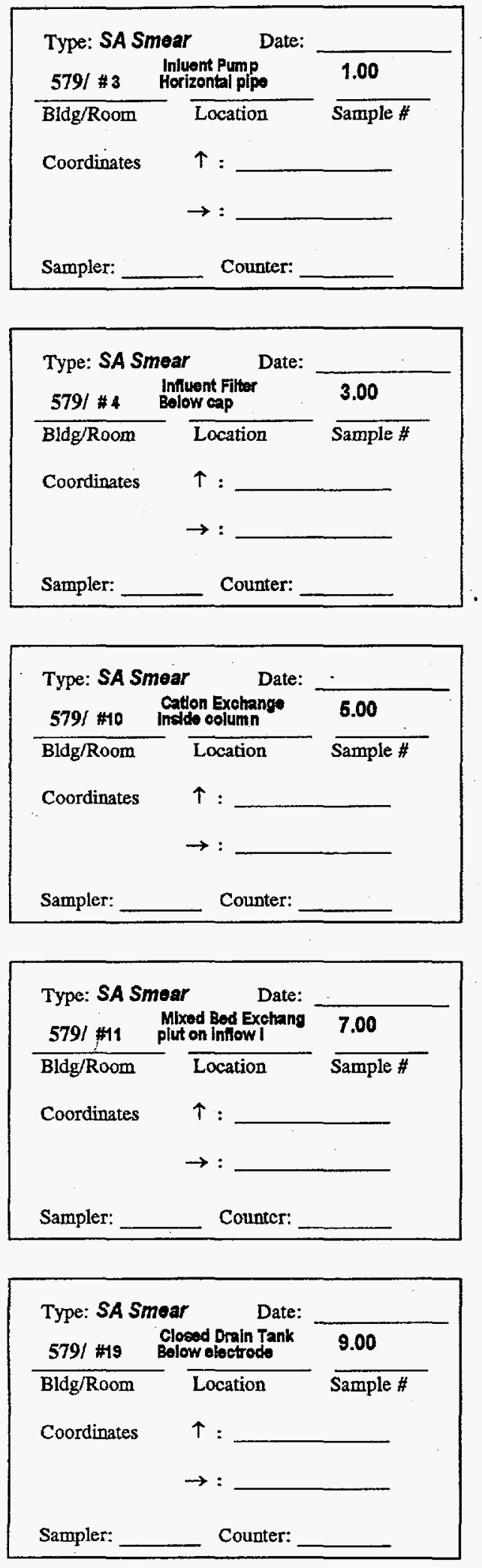
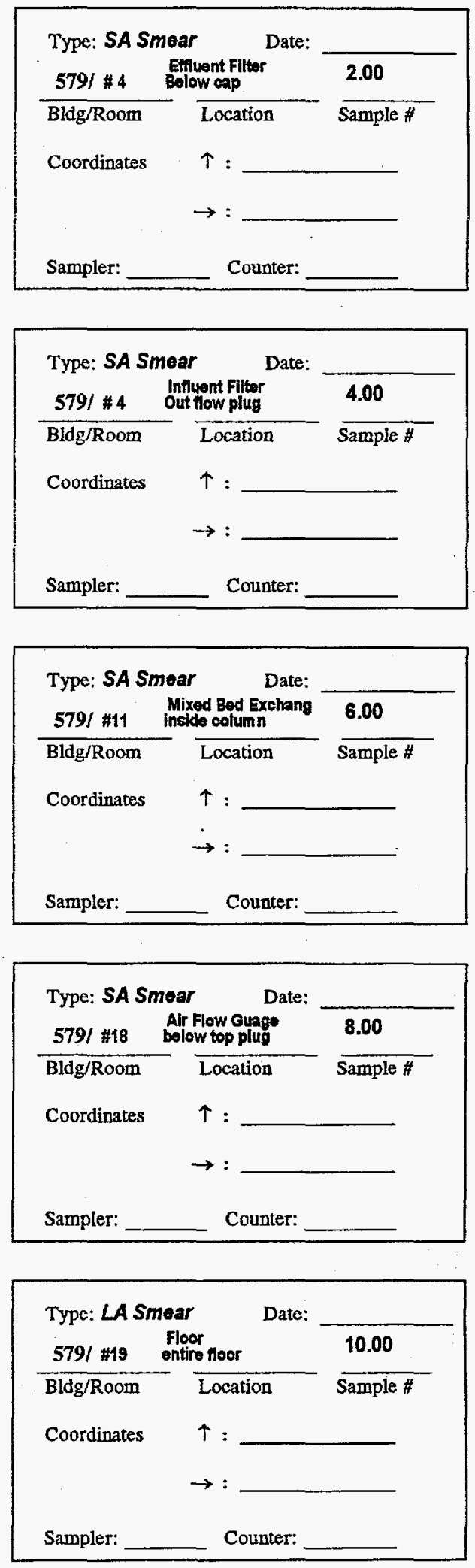

\section{FIGURE A1. Sample Labels}


TABLE A1. Building 579 Characterization Sample Labels

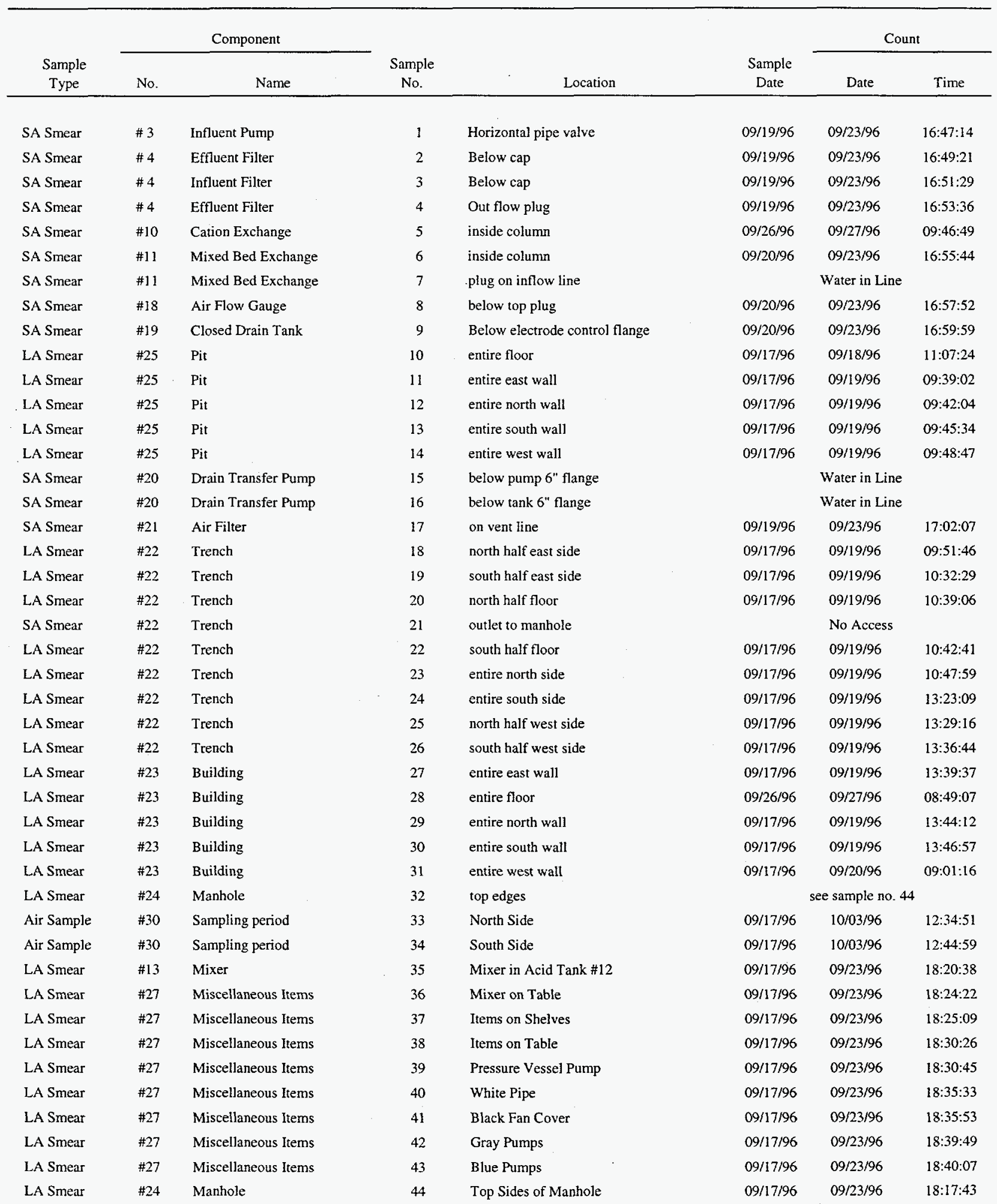


TABLE A1. (Cont.)

\begin{tabular}{|c|c|c|c|c|c|c|c|}
\hline \multirow{2}{*}{$\begin{array}{c}\text { Sample } \\
\text { Type }\end{array}$} & \multicolumn{2}{|r|}{ Component } & \multirow{2}{*}{$\begin{array}{c}\text { Sample } \\
\text { No. }\end{array}$} & \multirow[b]{2}{*}{ Location } & \multirow{2}{*}{$\begin{array}{c}\text { Sample } \\
\text { Date }\end{array}$} & \multicolumn{2}{|c|}{ Count } \\
\hline & No. & Name & & & & Date & Time \\
\hline Water 6469 & $\# 4$ & Influent Filter & 45 & Influent filter drain & $09 / 18 / 96$ & $09 / 25 / 96$ & \\
\hline Water 6470 & $\# 10$ & Cation Exchange & 46 & Column lower drain & $09 / 18 / 96$ & $09 / 25 / 96$ & \\
\hline Water 6471 & $\# 11$ & Mixed Bed Exchange & 47 & Column lower drain & $09 / 18 / 96$ & $09 / 25 / 96$ & \\
\hline Water 6472 & \#11 & Mixed Bed Exchange & 48 & Column upper drain & $09 / 18 / 96$ & $09 / 25 / 96$ & \\
\hline Water & \#4 & Effluent Filter & 49 & Effluent filter Drain & $09 / 18 / 96$ & $09 / 20 / 96$ & $05: 51: 43$ \\
\hline Water 6474 & $\# 24$ & Manhole & 50 & Top of Manhole & $09 / 18 / 96$ & $09 / 26 / 96$ & \\
\hline Q-tip Smear & $\# 4$ & Influent Filter & 51 & Influent filter (bottom) & $09 / 19 / 96$ & $09 / 23 / 96$ & $17: 04: 14$ \\
\hline Q-tip Smear & $\# 4$ & Influent Filter & 52 & Effluent filter (bottom) & $09 / 19 / 96$ & $09 / 23 / 96$ & $17: 06: 22$ \\
\hline SA Smear & \#26 & Effluent Pipe Outlet (east) & 53 & Exterior North Wall & $09 / 19 / 96$ & $09 / 23 / 96$ & 17:08:30 \\
\hline SA Smear & $\# 26$ & Effluent Pipe Outlet (west) & 54 & Exterior North Wall & $09 / 19 / 96$ & $09 / 23 / 96$ & $17: 10: 38$ \\
\hline Scrapings & \#24 & Manhole & 55 & Manhole (top) & $09 / 19 / 96$ & $09 / 23 / 96$ & $17: 12: 45$ \\
\hline SA Smear & \#19 & Closed Drain Tank & 56 & Drain Tank under vent pipe & $09 / 20 / 96$ & $09 / 23 / 96$ & $17: 14: 53$ \\
\hline Water 6417 & $\# 10$ & Cation Exchange & 57 & Inside column & $09 / 24 / 96$ & $09 / 26 / 96$ & \\
\hline Resin & $\# 10$ & Cation Exchange & 58 & Inside column & $09 / 20 / 96$ & $09 / 25 / 96$ & \\
\hline Water & \#11 & Mixed Bed Exchange & 59 & Inside column & $09 / 20 / 96$ & $09 / 25 / 96$ & \\
\hline Water & $\# 20$ & Drain tank & 60 & Drain Transfer Pump & $09 / 20 / 96$ & $09 / 25 / 96$ & \\
\hline SA Smear & $\# 16$ & Mixer & 61 & Mixer in Caustic Tank \#15 & $09 / 26 / 96$ & $09 / 27 / 96$ & $09: 48: 56$ \\
\hline SA Smear & $\# 13$ & Mixer & 62 & Mixer in Acid Tank \#12 & $09 / 26 / 96$ & $09 / 27 / 96$ & $09: 51: 04$ \\
\hline LA Smear & \#12 & Acid Regenerant Tank & 63 & Outside of Tank & $09 / 26 / 96$ & $09 / 27 / 96$ & $08: 54: 14$ \\
\hline LA Smear & $\# 12$ & Acid Regenerant Tank & 64 & Inside of Tank & $09 / 26 / 96$ & $09 / 27 / 96$ & $08: 59: 58$ \\
\hline LA Smear & $\# 15$ & Caustic Regenerant Tank & 65 & Outside of Tank & $09 / 26 / 96$ & $09 / 27 / 96$ & $09: 30: 34$ \\
\hline LA Smear & $\# 15$ & Caustic Regenerant Tank & 66 & Inside of Tank & $09 / 26 / 96$ & $09 / 27 / 96$ & $09: 37: 54$ \\
\hline Water 6414 & $\# 24$ & Manhole & 67 & Inside manhole & $09 / 25 / 96$ & $09 / 26 / 96$ & \\
\hline SA Smear & $\# 29$ & Influent Pipe Inlet & 68 & Exterior South Wall & $10 / 02 / 96$ & $10 / 03 / 96$ & $12: 49: 52$ \\
\hline LA Smear & $\# 23$ & Floor & 601 & Water from bucket under Influent Filter & $09 / 17 / 96$ & $09 / 18 / 96$ & 09:07:41 \\
\hline
\end{tabular}



APPENDIX B: Smear Sample Data 

TABLE B1. Composite Smear and Scraping Data for Building 579 (594)

\begin{tabular}{|c|c|c|c|c|c|c|}
\hline \multirow[b]{2}{*}{$\begin{array}{c}\text { Sample } \\
\text { Type }\end{array}$} & \multicolumn{2}{|r|}{ Component } & \multirow[b]{2}{*}{$\begin{array}{c}\text { Sample } \\
\text { No. }\end{array}$} & \multirow[b]{2}{*}{ Location } & \multicolumn{2}{|c|}{$\mathrm{dis} / \mathrm{min}$} \\
\hline & No & Name & & & Alpha & Beta \\
\hline SA Smear. & $\# 3$ & Influent Pump & 1 & Horizontal pipe valve & $3.44 \pm 5.78$ & $-8.4 \pm 15.6$ \\
\hline SA Smear & $\# 4$ & Effluent Filter & 2 & Below cap & $7.60 \pm 8.17$ & $21.3 \pm 19.5$ \\
\hline SA Smear & $\# 4$ & Effluent Filter & 4 & Out flow plug & $3.44 \pm 5.78$ & $2.3 \pm 17.1$ \\
\hline Water & $\# 4$ & Effluent Filter & 49 & Effluent filter Drain & $0.46 \pm 0.26$ & $1.1 \pm 1.8$ \\
\hline SA Smear & $\# 4$ & Influent Filter & 3 & Below cap & $3.44 \pm 5.78$ & $5.8 \pm 17.5$ \\
\hline Q-tip Smear & $\# 4$ & Influent Filter & 51 & Influent filter (bottom) & $-0.73 \pm 4.09$ & $8.2 \pm 17.8$ \\
\hline Q-tip Smear & $\# 4$ & Influent Filter & 52 & Effluent filter (bottom) & $-0.73 \pm 4.09$ & $-6.1 \pm 15.9$ \\
\hline SA Smear & $\# 10$ & Cation Exchange & 5 & inside column & $28.50 \pm 15.28$ & $105.1 \pm 27.7$ \\
\hline SA Smear & $\# 11$ & Mixed Bed Exchange & 6 & inside column & $13.85 \pm 10.81$ & $5.8 \pm 17.5$ \\
\hline LA Smear & $\# 12$ & Acid Regenerant Tank & 63 & Outside of Tank & $3.1 \pm 7.8$ & $44.4 \pm 47.1$ \\
\hline LA Smear & $\# 12$ & Acid Regenerant Tank & 64 & Inside of Tank & $7.8 \pm 9.5$ & $171.8 \pm 52.1$ \\
\hline LA Smear & $\# 13$ & Lead Grip Agitator & 35 & Mixer in Acid Tank \#12 & $9.4 \pm 10.0$ & $83.6 \pm 48.7$ \\
\hline SA Smear & $\# 13$ & Lead Grip Agitator & 62 & Mixer in Tank \#12 & $-0.67 \pm 4.09$ & $11.1 \pm 18.3$ \\
\hline LA Smear & $\# 15$ & Caustic Regenerant Tank & 65 & Outside of Tank & $1.5 \pm 7.1$ & $58.1 \pm 47.7$ \\
\hline LA Smear & $\# 15$ & Caustic Regenerant Tank & 66 & Inside of Tank & $6.2 \pm 9.0$ & $26.7 \pm 46.4$ \\
\hline SA Smear & \#16 & Caustic Mixer & 61 & Mixer in Tank \#15 & $-0.67 \pm 4.09$ & $-11.6 \pm 15.2$ \\
\hline SA Smear & $\# 18$ & Air Flow Gauge & 8 & below top plug & $11.77 \pm 10.00$ & $2.3 \pm 17.1$ \\
\hline SA Smear & $\# 19$ & Closed Drain Tank & 9 & Below electrode control flange & $-0.73 \pm 4.09$ & $11.8 \pm 18.3$ \\
\hline SA Smear & $\# 19$ & Closed Drain Tank & 56 & Drain Tank under vent pipe & $105.52 \pm 29.16$ & $47.5 \pm 22.3$ \\
\hline SA Smear & $\# 21$ & Air Filter & 17 & on vent line & $-0.73 \pm 4.09$ & $2.3 \pm 17.1$ \\
\hline LA Smear & $\# 22$ & Trench & 18 & north half east side & $3.1 \pm 7.8$ & $-9.5 \pm 44.8$ \\
\hline LA Smear & $\# 22$ & Trench & 19 & south half east side & $-0.1 \pm 6.4$ & $2.2 \pm 45.3$ \\
\hline LA Smear & $\# 22$ & Trench & 20 & north half floor & $3.1 \pm 7.8$ & $44.4 \pm 47.1$ \\
\hline LA Smear & $\# 22$ & Trench & 22 & south half floor & $1.5 \pm 7.1$ & $3.2 \pm 45.4$ \\
\hline LA Smear & $\# 22$ & Trench & 23 & entire north side & $-1.7 \pm 5.5$ & $27.7 \pm 46.4$ \\
\hline LA Smear & $\# 22$ & Trench & 24 & entire south side & $6.2 \pm 9.0$ & $24.8 \pm 46.3$ \\
\hline LA Smear & $\# 22$ & Trench & 25 & north half west side & $12.5 \pm 11.0$ & $42.4 \pm 47.0$ \\
\hline LA Smear & $\# 22$ & Trench & 26 & south half west side & $6.2 \pm 9.0$ & $-2.7 \pm 45.1$ \\
\hline LA Smear & $\# 23$ & Building & 27 & entire east wall & $6.2 \pm 9.0$ & $37.5 \pm 46.8$ \\
\hline LA Smear & $\# 23$ & Building & 28 & entire floor & $3.1 \pm 7.8$ & $58.1 \pm 47.7$ \\
\hline LA Smear & $\# 23$ & Building & 29 & entire north wall & $-0.1 \pm 6.4$ & $27.7 \pm 46.4$ \\
\hline LA Smear & $\# 23$ & Building & 30 & entire south wall & $-0.1 \pm 6.4$ & $8.1 \pm 45.6$ \\
\hline LA Smear & $\# 23$ & Building & 31 & entire west wall & $12.5 \pm 11.0$ & $75.7 \pm 48.4$ \\
\hline LA Smear & $\# 23$ & Floor & 601 & Water from bucket under Influent Filter & $-0.7 \pm 6.4$ & $-46.8 \pm 44.6$ \\
\hline LA Smear & $\# 24$ & Manbole & 44 & Top Sides of Manhole & $3.1 \pm 7.8$ & $86.5 \pm 48.8$ \\
\hline Scrapings & $\# 24$ & Manhole & 55 & Manhole (top) & $1.35 \pm 4.09$ & $1.1 \pm 16.9$ \\
\hline LA Smear & $\# 25$ & Pit & 10 & entire floor & $-3.3 \pm 4.6$ & $49.3 \pm 47.3$ \\
\hline LA Smear & $\# 25$ & Pit & 11 & entire east wall & $-1.7 \pm 5.5$ & $43.4 \pm 47.1$ \\
\hline LA Smear & $\# 25$ & Pit & 12 & entire north wall & $-0.1 \pm 6.4$ & $19.9 \pm 46.1$ \\
\hline LA Smear & $\# 25$ & Pit & 13 & entire south wall & $4.6 \pm 8.4$ & $42.4 \pm 47.0$ \\
\hline LA Smear & $\# 25$ & Pit & 14 & entire west wall & $7.8 \pm 9.5$ & $34.6 \pm 46.7$ \\
\hline SA Smear & $\# 26$ & Effluent Pipe Outlet (east) & 53 & Exterior North Wall & $3.44 \pm 5.78$ & $2.3 \pm 17.1$ \\
\hline SA Smear & $\# 26$ & Effluent Pipe Outlet (west) & 54 & Exterior North Wall & $5.52 \pm 7.08$ & $24.9 \pm 19.9$ \\
\hline LA Smear & $\# 27$ & Miscellaneous Items & 36 & Mixer on Table & $4.8 \pm 9.0$ & $52.7 \pm 48.9$ \\
\hline
\end{tabular}


March 19, 1997

Page B-4

TABLE B1. (Cont.)

\begin{tabular}{|c|c|c|c|c|c|c|}
\hline \multirow[b]{2}{*}{$\begin{array}{c}\text { Sample } \\
\text { Type }\end{array}$} & \multicolumn{2}{|r|}{ Component } & \multirow[b]{2}{*}{$\begin{array}{c}\text { Sample } \\
\text { No. }\end{array}$} & & \multicolumn{2}{|c|}{$\mathrm{dis} / \mathrm{min}$} \\
\hline & No & Name & & Location & Alpha & Beta \\
\hline LA Smear & $\# 27$ & Miscellaneous Items & 37 & Items on Shelves & $3.1 \pm 7.8$ & $-9.5 \pm 44.8$ \\
\hline LA Smear & $\# 27$ & Miscellaneous Items & 38 & Items on Table & $-2.6 \pm 5.3$ & $-17.7 \pm 45.9$ \\
\hline LA Smear & $\# 27$ & Miscellaneous Items & 39 & Pressure Vessel Pump & $1.5 \pm 7.1$ & $49.3 \pm 47.3$ \\
\hline LA Smear & $\# 27$ & Miscellaneous Items & 40 & White Pipe & $4.8 \pm 9.0$ & $-19.7 \pm 45.8$ \\
\hline LA Smear & $\# 27$ & Miscellaneous Items & 41 & Black Fan Cover & $7.8 \pm 9.5$ & $22.8 \pm 46.2$ \\
\hline LA Smear & $\# 27$ & Miscellaneous Items & 42 & Grey Pumps & $-0.7 \pm 6.4$ & $36.6 \pm 48.2$ \\
\hline LA Smear & $\# 27$ & Miscellaneous Items & 43 & Blue Pumps & $7.8 \pm 9.5$ & $45.3 \pm 47.2$ \\
\hline \multirow[t]{6}{*}{ SA Smear } & $\# 29$ & Influent Pipe Inlet & 68 & Exterior South Wall & $1.33 \pm 4.09$ & $2.3 \pm 17.4$ \\
\hline & & & & Minimum & $-3.30 \pm 0.26$ & $-46.8 \pm 1.8$ \\
\hline & & & & Maximum & $105.52 \pm 29.16$ & $171.8 \pm 52.1$ \\
\hline & & & & Average & $5.81 \pm 7.62$ & $25.8 \pm 36.1$ \\
\hline & & & & Standard Deviation & $14.95 \pm 3.91$ & $35.9 \pm 14.5$ \\
\hline & & & & Count & 52 & 52 \\
\hline
\end{tabular}


TABLE B2. Smear Scraping and Water Data from Tennelec for Building 579 (594)

\begin{tabular}{|c|c|c|c|c|c|c|}
\hline \multirow[b]{2}{*}{ Sample Type } & \multicolumn{2}{|r|}{ Component } & \multirow{2}{*}{$\begin{array}{c}\text { Sample } \\
\text { No. }\end{array}$} & \multirow[b]{2}{*}{ Location } & \multicolumn{2}{|c|}{$\mathrm{dis} / \mathrm{min}$} \\
\hline & No & Name & & & Alpha & Beta \\
\hline SA Smear & $\# 3$ & Influent Pump & 1 & Horizontal pipe valve & $3.44 \pm 5.78$ & $-8.4 \pm 15.6$ \\
\hline SA Smear & $\# 4$ & Effluent Filter & 2 & Below cap & $7.60 \pm 8.17$ & $21.3 \pm 19.5$ \\
\hline SA Smear & $\# 4$ & Influent Filter & 3 & Below cap & $3.44 \pm 5.78$ & $5.8 \pm 17.5$ \\
\hline SA Smear & $\# 4$ & Effluent Filter & 4 & Out flow plug & $3.44 \pm 5.78$ & $2.3 \pm 17.1$ \\
\hline Water & $\# 4$ & Effluent Filter & 49 & Effluent filter Drain & $0.46 \pm 0.26$ & $1.1 \pm 1.8$ \\
\hline Q-tip Smear & $\# 4$ & Influent Filter & 51 & Influent filter (bottom) & $-0.73 \pm 4.09$ & $8.2 \pm 17.8$ \\
\hline Q-tip Smear & $\# 4$ & Influent Filter & 52 & Effluent filter (bottom) & $-0.73 \pm 4.09$ & $-6.1 \pm 15.9$ \\
\hline SA Smear & $\# 10$ & Cation Exchange & 5 & inside column & $28.50 \pm 15.28$ & $105.1 \pm 27.7$ \\
\hline SA Smear & $\# 11$ & Mixed Bed Exchange & 6 & inside column & $13.85 \pm 10.81$ & $5.8 \pm 17.5$ \\
\hline SA Smear & $\# 13$ & Mixer & 62 & Mixer in Tank $\# 12$ & $-0.67 \pm 4.09$ & $11.1 \pm 18.3$ \\
\hline SA Smear & $\# 16$ & Mixer & 61 & Mixer in Tank \#15 & $-0.67 \pm 4.09$ & $-11.6 \pm 15.2$ \\
\hline SA Smear & $\# 18$ & Air Flow Gauge & 8 & below top plug & $11.77 \pm 10.00$ & $2.3 \pm 17.1$ \\
\hline SA Smear & $\# 19$ & Closed Drain Tank & 9 & Below electrode control flange & $-0.73 \pm 4.09$ & $11.8 \pm 18.3$ \\
\hline SA Smear & $\# 19$ & Closed Drain Tank & 56 & Drain Tank under vent pipe & $105.52 \pm 29.16$ & $47.5 \pm 22.3$ \\
\hline SA Smear & $\# 21$ & Air Filter & 17 & on vent line & $-0.73 \pm 4.09$ & $2.3 \pm 17.1$ \\
\hline Scrapings & $\# 24$ & Manhole & 55 & Manhole (top) & $1.35 \pm 4.09$ & $1.1 \pm 16.9$ \\
\hline SA Smear & $\# 26$ & Effluent Pipe Outlet (east) & 53 & Exterior North Wall & $3.44 \pm 5.78$ & $2.3 \pm 17.1$ \\
\hline SA Smear & $\# 26$ & Effluent Pipe Outlet (west) & 54 & Exterior North Wall & $5.52 \pm 7.08$ & $24.9 \pm 19.9$ \\
\hline \multirow[t]{6}{*}{ SA Smear } & $\# 29$ & Influent Pipe Inlet & 68 & Exterior South Wall & $1.33 \pm 4.09$ & $2.3 \pm 17.4$ \\
\hline & & & & Minimum & $-0.73 \pm 0.26$ & $-11.6 \pm 1.8$ \\
\hline & & & & Maximum & $105.52 \pm 29.16$ & $105.1 \pm 27.7$ \\
\hline & & & & Average & $9.76 \pm 7.19$ & $12.1 \pm 17.4$ \\
\hline & & & & Standard Deviation & $23.63 \pm 6.08$ & $25.4 \pm 4.6$ \\
\hline & & & & Count & 19 & 19 \\
\hline
\end{tabular}


TABLE B3. Building 579 Smear Data logged on the DABRAS or NE

\begin{tabular}{|c|c|c|c|c|c|c|}
\hline \multirow{2}{*}{$\begin{array}{c}\text { Sample } \\
\text { Type }\end{array}$} & \multicolumn{2}{|r|}{ Component } & \multirow{2}{*}{$\begin{array}{c}\text { Sample } \\
\text { No. }\end{array}$} & & \multicolumn{2}{|c|}{$\mathrm{dis} / \mathrm{min}$} \\
\hline & No & Name & & Location & Alpha & Beta \\
\hline LA Smear & $\# 12$ & Acid Regenerant Tank & 63 & Outside of Tank & $3.1 \pm 7.8$ & $44.4 \pm 47.1$ \\
\hline LA Smear & $\# 12$ & Acid Regenerant Tank & 64 & Inside of Tank & $7.8 \pm 9.5$ & $171.8 \pm 52.1$ \\
\hline LA Smear & $\# 13$ & Mixer & 35 & Mixer in Acid Tank \#12 & $9.4 \pm 10.0$ & $83.6 \pm 48.7$ \\
\hline LA Smear & $\# 15$ & Caustic Regenerant Tank & 65 & Outside of Tank & $1.5 \pm 7.1$ & $58.1 \pm 47.7$ \\
\hline LA Smear & $\# 15$ & Caustic Regenerant Tank & 66 & Inside of Tank & $6.2 \pm 9.0$ & $26.7 \pm 46.4$ \\
\hline LA Smear & $\# 22$ & Trench & 18 & north half east side & $3.1 \pm 7.8$ & $-9.5 \pm 44.8$ \\
\hline LA Smear & $\# 22$ & Trench & 19 & south half east side & $-0.1 \pm 6.4$ & $2.2 \pm 45.3$ \\
\hline LA Smear & \#22 & Trench & 20 & north half floor & $3.1 \pm 7.8$ & $44.4 \pm 47.1$ \\
\hline LA Smear & $\# 22$ & Trench & 22 & south half floor & $1.5 \pm 7.1$ & $3.2 \pm 45.4$ \\
\hline LA Smear & $\# 22$ & Trench & 23 & entire north side & $-1.7 \pm 5.5$ & $27.7 \pm 46.4$ \\
\hline LA Smear & $\# 22$ & Trench & 24 & entire south side & $6.2 \pm 9.0$ & $24.8 \pm 46.3$ \\
\hline LA Smear & $\# 22$ & Trench & 25 & north half west side & $12.5 \pm 11.0$ & $42.4 \pm 47.0$ \\
\hline LA Smear & $\# 22$ & Trench & 26 & south half west side & $6.2 \pm 9.0$ & $-2.7 \pm 45.1$ \\
\hline LA Smear & $\# 23$ & Building & 27 & entire east wall & $6.2 \pm 9.0$ & $37.5 \pm 46.8$ \\
\hline LA Smear & $\# 23$ & Building & 28 & entire floor & $3.1 \pm 7.8$ & $58.1 \pm 47.7$ \\
\hline LA Smear & $\# 23$ & Building & 29 & entire north wall & $-0.1 \pm 6.4$ & $27.7 \pm 46.4$ \\
\hline LA Smear & $\# 23$ & Building & 30 & entire south wall & $-0.1 \pm 6.4$ & $8.1 \pm 45.6$ \\
\hline LA Smear & $\# 23$ & Building & 31 & entire west wall & $12.5 \pm 11.0$ & $75.7 \pm 48.4$ \\
\hline LA Smear & $\# 23$ & Floor & 601 & Water from bucket under Influent Filter & $-0.7 \pm 6.4$ & $-46.8 \pm 44.6$ \\
\hline LA Smear & $\# 24$ & Manhole & 44 & Top Sides of Manhole & $3.1 \pm 7.8$ & $86.5 \pm 48.8$ \\
\hline LA Smear & \#25 & Pit & 10 & entire floor & $-3.3 \pm 4.6$ & $49.3 \pm 47.3$ \\
\hline LA Smear & $\# 25$ & Pit & 11 & entire east wall & $-1.7 \pm 5.5$ & $43.4 \pm 47.1$ \\
\hline LA Smear & $\# 25$ & Pit & 12 & entire north wall & $-0.1 \pm 6.4$ & $19.9 \pm 46.1$ \\
\hline LA Smear & $\# 25$ & Pit & 13 & entire south wall & $4.6 \pm 8.4$ & $42.4 \pm 47.0$ \\
\hline LA Smear & $\# 25$ & Pit & 14 & entire west wall & $7.8 \pm 9.5$ & $34.6 \pm 46.7$ \\
\hline LA Smear & $\# 27$ & Miscellaneous Items & 36 & Mixer on Table & $4.8 \pm 9.0$ & $52.7 \pm 48.9$ \\
\hline LA Smear & $\# 27$ & Miscellaneous Items & 37 & Items on Shelves & $3.1 \pm 7.8$ & $-9.5 \pm 44.8$ \\
\hline LA Smear & $\# 27$ & Miscellaneous Items & 38 & Items on Table & $-2.6 \pm 5.3$ & $-17.7 \pm 45.9$ \\
\hline LA Smear & $\# 27$ & Miscellaneous Items & 39 & Pressure Vessel Pump & $1.5 \pm 7.1$ & $49.3 \pm 47.3$ \\
\hline LA Smear & $\# 27$ & Miscellaneous Items & 40 & White Pipe & $4.8 \pm 9.0$ & $-19.7 \pm 45.8$ \\
\hline LA Smear & $\# 27$ & Miscellaneous Items & 41 & Black Fan Cover & $7.8 \pm 9.5$ & $22.8 \pm 46.2$ \\
\hline LA Smear & $\# 27$ & Miscellaneous Items & 42 & Grey Pumps & $-0.7 \pm 6.4$ & $36.6 \pm 48.2$ \\
\hline \multirow[t]{6}{*}{ LA Smear } & $\# 27$ & Miscellaneous Items & 43 & Blue Pumps & $7.8 \pm 9.5$ & $45.3 \pm 47.2$ \\
\hline & & & & Minimum & $-3.3 \pm 4.6$ & $-47 \pm 45$ \\
\hline & & & & Maximum & $12.5 \pm 11.0$ & $172 \pm 52$ \\
\hline & & & & Average & $3.5 \pm 7.9$ & $34 \pm 47$ \\
\hline & & & & Standard Deviation & $4.1 \pm 1.6$ & $38 \pm 1$ \\
\hline & & & & Count & 33 & 33 \\
\hline
\end{tabular}


APPENDIX C: Air and Water Sample Data 

TABLE C1. Air Sample Data from Tennelec for Building 579 (594)

\begin{tabular}{|c|c|c|c|c|c|c|c|c|c|}
\hline \multirow{2}{*}{$\begin{array}{l}\text { Sample } \\
\text { Type }\end{array}$} & \multicolumn{2}{|r|}{ Component } & \multirow{2}{*}{$\begin{array}{c}\text { Sample } \\
\text { No. }\end{array}$} & \multirow[b]{2}{*}{ Location } & \multirow{2}{*}{$\begin{array}{l}\text { Date } \\
\text { Started }\end{array}$} & \multirow{2}{*}{$\begin{array}{c}\text { Date } \\
\text { Removed }\end{array}$} & \multicolumn{2}{|c|}{ initial dis/min } & \multirow{2}{*}{$\begin{array}{l}\text { Date } \\
\text { Count }\end{array}$} \\
\hline & No. & Name & & & & & Alpha & Beta & \\
\hline Air & $\# 30$ & Sampling period & 1 & North Side & $09 / 17 / 96$ & $09 / 27 / 96$ & $4.67 \pm 2.95$ & $37.3 \pm 9.7$ & $10 / 03 / 96$ \\
\hline \multirow[t]{6}{*}{ Air } & $\# 30$ & Sampling period & 2 & South Side & $09 / 17 / 96$ & $09 / 27 / 96$ & $1.75 \pm 2.02$ & $31.8 \pm 9.5$ & $10 / 03 / 96$ \\
\hline & & & & & & Minimum & $1.75 \pm 2.02$ & $31.8 \pm 9.5$ & \\
\hline & & & & & & Maximum & $4.67 \pm 2.95$ & $37.3 \pm 9.7$ & \\
\hline & & & & & & Average & $3.21 \pm 2.49$ & $34.6 \pm 9.6$ & \\
\hline & & & & & Stan & d Deviation & $1.46 \pm 0.47$ & $2.7 \pm 0.1$ & \\
\hline & & & & & & Count & 2 & 2 & \\
\hline
\end{tabular}

Working Volume $\left(\mathrm{m}^{3}\right)=40$ liters $/ \mathrm{min} \times$ time (time $=60 \mathrm{~min} / \mathrm{hr} \times 8$ hours $\mathrm{x}$ number of week days $)$

Alpha DAC $=6$ for ${ }^{239} \mathrm{Pu},{ }^{241} \mathrm{Am} \&{ }^{238} \mathrm{Pu}$

Beta DAC $=4,000$ for ${ }^{90} \mathrm{Sr}$

TABLE C2. Building 579 Characterization Water Samples

\begin{tabular}{|c|c|c|c|c|c|c|c|}
\hline \multirow{2}{*}{$\begin{array}{c}\text { Sample } \\
\text { Type }\end{array}$} & \multirow{2}{*}{$\begin{array}{c}\text { Analysis } \\
\text { No. }\end{array}$} & \multicolumn{2}{|r|}{ Component } & \multicolumn{2}{|r|}{ Sample } & \multicolumn{2}{|c|}{$\mathrm{dis} / \mathrm{min} / \mathrm{mL}$} \\
\hline & & No. & Name & No. & Location & Alpha & Beta \\
\hline Water & 6469 & $\# 4$ & Influent Filter & 45 & Influent filter drain & 0.069 & 0.218 \\
\hline Water & 6470 & $\# 10$ & Cation Exchange & 46 & Column lower drain & 0.04 & 0.324 \\
\hline Water & 6471 & $\# 11$ & Mixed Bed Exchange & 47 & Column lower drain & 0.044 & 0.871 \\
\hline Water & 6472 & $\# 11$ & Mixed Bed Exchange & 48 & Column upper drain & 0.039 & 0.486 \\
\hline Water & 6474 & $\# 24$ & Manhole & 50 & Top of Manhole & 0.03 & 0.1 \\
\hline Water & 6417 & $\# 10$ & Cation Exchange & 57 & Inside column & 0.03 & 0.004 \\
\hline Resin & $96-0483-01$ & $\# 10$ & Cation Exchange & 58 & Inside column & 19.2 & 4.3 \\
\hline Water & $96-0484-01$ & \#11 & Mixed Bed Exchange & 59 & Inside column & $<0.09$ & $<0.16$ \\
\hline Water & $96-0484-02$ & $\# 20$ & Drain Transfer Pump & 60 & Drain tank & $<0.09$ & $<0.16$ \\
\hline \multirow[t]{6}{*}{ Water } & 6414 & $\# 24$ & Manhole & 67 & Inside manhole & 0.37 & 0.99 \\
\hline & & & & & Minimum & 0.030 & 0.004 \\
\hline & & & & & Maximum & 19.2 & 4.3 \\
\hline & & & & & Average & 2.000 & 0.761 \\
\hline & & & & & Standard Deviation & 5.734 & 1.220 \\
\hline & & & & & Count & 10 & 10 \\
\hline
\end{tabular}


APPENDIX D: Analytical Results 


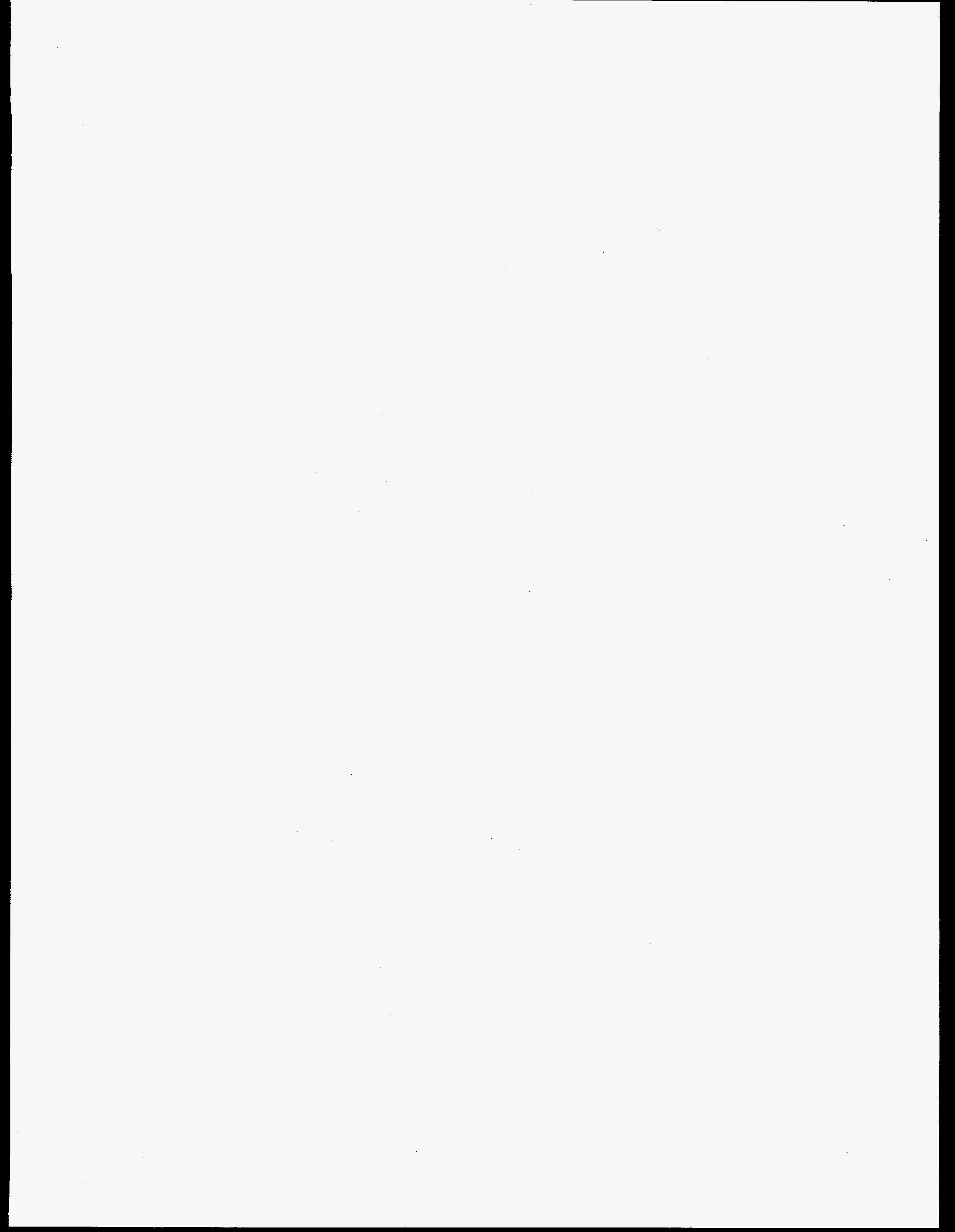


TABLE D1. Documents Containing Analytical Results

\begin{tabular}{ll}
\hline Identification & Page No. \\
\hline & \\
Control Lab analysis Results of Water & D-5 \\
Argonne ACL Resin and Water pH Results & D-16 \\
Argonne ACL Resin and Water Metals Results & D-17 \\
Argonne ACL Resin and Water Radionuclide Results & D-23 \\
Argonne ACL Resin and Water Volatile Organic Results & D-26 \\
Argonne ACL Resin and Water Semivolatile Organic Results & D-37 \\
\hline
\end{tabular}


TABLE D2. Water and Resin from Exchange Vessels

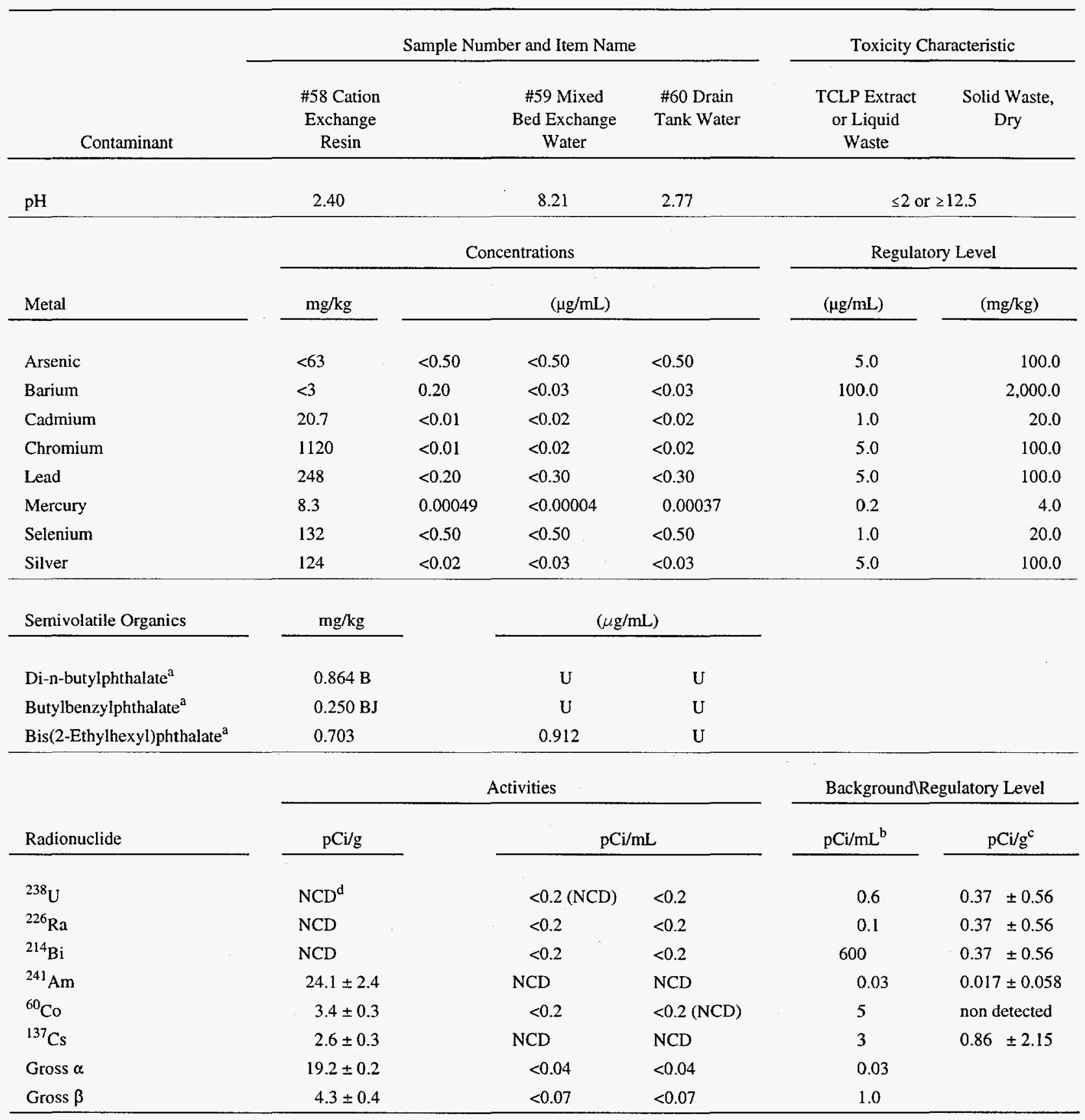

a See original data for definition of the qualifiers.

b DOE 5400.5 Derived Concentration Guide (DCG).

c ANL Perimeter Bottom Sediment Averages from Table 2.

d No Contamination Detected. 


\section{REQUEST FOR ANALYSIS $\quad 6470$}

Date: $9 / 18 / 96$ Bldg: 579 CL Sample: $\$ 6$ Type of Sample: Water - Cation Exchang-Columa Gallons: $6 \mathrm{cy}$. Requestor: Chamelte thalaw

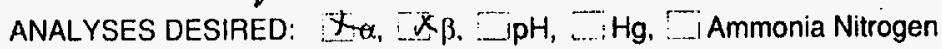
- Total Solids, -... Volume for $20 \%$ Bottoms

Other:

Date Analyzed: 9-25-96 Analyst: fai da cal Results: $\chi-0.040 \mathrm{~d} / \mathrm{m} / \mathrm{m} /$.

$$
3-0.324 \mathrm{~d} / \mathrm{m} / \mathrm{m} / \text {. }
$$

\begin{tabular}{|c|c|c|c|c|}
\hline Size & Determination & Reading & Result & \\
\hline & & & & \\
\hline & & & & \\
\hline & & & & \\
\hline & & & & \\
\hline & & & & \\
\hline & & & & \\
\hline
\end{tabular}

\begin{tabular}{|l|l|l|}
\hline & Total Solids & Total Solids \\
\hline Dish Number & & \\
\hline Wt. Dish + Wet Sample & & \\
\hline Wt. Dish & & \\
\hline Wt. Sample & & \\
\hline Wt. Dish + Dry Sample & & \\
\hline Wt. Dish & & \\
\hline Wt. Dry Sample & & \\
\hline Remarks and Calculations: & \\
\end{tabular}

\section{REQUEST FOR ANALYSIS}

6469

Date: $9 / 18 / 96$ Bldg: 529 CL. Sample: 45

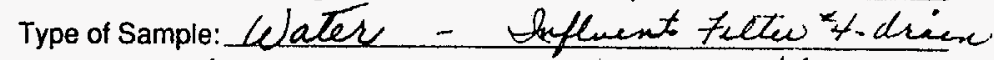
Gallons: 6 g. Requestor: Churlate threen ANALYSES DESIRED: $\mp \alpha, X_{i} \beta .-\mathrm{pH}, \ldots \mathrm{Hg}, \cdots$ Ammonia Nitrogen $\square$ Total Solids, $\square$ volume for $20 \%$ Bottoms

Other:

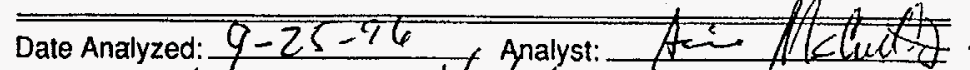
Results: $\alpha \geq 0.069 d / a_{1} / \mathrm{m} /$.

$$
3-0.218 \mathrm{~d} / \mathrm{m} / \mathrm{m} / \mathrm{.}
$$

\begin{tabular}{|c|c|c|c|}
\hline Size & Determination & Reading & Result \\
\hline & & & \\
\hline & & & \\
\hline & & & \\
\hline & & & \\
\hline & & & \\
\hline & & & \\
\hline
\end{tabular}

\begin{tabular}{|l|l|l|}
\hline \hline & Total Solids & Total Solids \\
\hline Dish Number & & \\
\hline Wt. Dish + Wet Sample & & \\
\hline Wt. Dish & & \\
\hline W. Sample & & \\
\hline Wt. Dish + Dry Sample & & \\
\hline W. Dish & & \\
\hline Wt. Dry Sample & & \\
\hline Remarks and Calculations: & & \\
& & \\
\end{tabular}


COUNTER DATA SHEET

sampie D-579 WATER. Intluertfilter \#4 DRAN volume 45 sample Date $9 / 18 / 96$ aPFS-11 QPO-97 Counted By Date Counted 9-25-96

\begin{tabular}{|c|c|c|c|c|c|c|c|}
\hline \multicolumn{3}{|c|}{ ALPHA-BETA COUNTING } & \multirow{2}{*}{\multicolumn{5}{|c|}{ SPIKED SAMPLE PERCENT RECOVERY DETERMINATION }} \\
\hline \multirow{2}{*}{$\begin{array}{c}\square \text { SPIKED } \\
\text { Counter Numbers } \\
\end{array}$} & \multicolumn{2}{|c|}{ 口 UNSPIKED } & & & & & \\
\hline & 17 & 18 & Counter Numbers & \multirow[b]{2}{*}{ ALPHA } & \multirow[b]{2}{*}{ BETA } & \multirow[b]{2}{*}{ ALPHA } & \multirow[b]{2}{*}{ BETA } \\
\hline Total $\alpha$ Counts & 8 & 16 & & & & & \\
\hline Counting Time & 30 & 3.0 & \multirow{2}{*}{$\begin{array}{l}\text { Standard Solution } \\
d / m / m \mid+50=\text { Known } \\
d / m / m \mid\end{array}$} & \multirow{2}{*}{$\overline{50}=$} & \multirow{2}{*}{$\overline{50}=$} & \multirow{2}{*}{$\overline{50}=$} & \multirow{2}{*}{$\overline{50}=$} \\
\hline Total $\alpha \mathrm{c} / \mathrm{m}$ & .27 & .53 & & & & & \\
\hline Background & .05 & .05 & & & & & \\
\hline Net $\alpha \mathrm{c} / \mathrm{m}$ & .22 & 48 & \multirow{2}{*}{$\begin{array}{l}\text { Spiked Sample } \alpha \text { \& } \beta \\
\text { Results in } \alpha / \mathrm{m} / \mathrm{ml}\end{array}$} & & & & \\
\hline mi Sample & 10 & 10 & & & & & \\
\hline$\alpha \mathrm{c} / \mathrm{m} / \mathrm{ml}$ & .022 & .048 & & & & & \\
\hline$\alpha$ Yield Factor & 1.96 & 1.96 & \multirow{2}{*}{\begin{tabular}{|l} 
Unspiked sample $\alpha \&$ \\
$\beta$ Results (from \\
PFS-11)
\end{tabular}} & & & & \\
\hline$\alpha d / m / m l$ & .043 & .094 & & & & & \\
\hline Total $\beta$ Counts & 1299 & 1297 & \multirow{2}{*}{$\begin{array}{l}\text { Spiked } d / \mathrm{m} / \mathrm{m} / \text { minus } \\
\text { Unspiked } d / \mathrm{m} / \mathrm{ml}= \\
\text { Recovered } \mathrm{d} / \mathrm{m} / \mathrm{ml}\end{array}$} & & & & \\
\hline Counting Time & $\frac{11}{30}$ & 30 & & & & & \\
\hline Total $\beta \mathrm{dm}$ & 46.63 & 46.40 & & & & & \\
\hline$\beta$ Background & 44,9 & 44.1 & \multirow{2}{*}{$\begin{array}{l}\text { Recovered d/m/ml }+ \\
\text { Known d/m/m/ } \times 100= \\
\text { Percent Recovery }\end{array}$} & & & & \multirow[b]{2}{*}{ - } \\
\hline $\mathrm{BKGD}+$ Net $\alpha \mathrm{c} / \mathrm{m}$ & $45 \cdot 12$ & 44.58 & & & & & \\
\hline Net $\beta c / m$ & 1.41 & 1.82 & & & & & \\
\hline mi Sample & 10 & 10 & \multicolumn{5}{|c|}{ REMARKS } \\
\hline$\beta \mathrm{c} / \mathrm{m} / \mathrm{ml}$ & .14 & .182 & & & & & \\
\hline$\beta$ Yield Factor & 1.35 & 1.35 & & & & & \\
\hline$\beta d / m / m l$ & .190 & .246 & & & & & \\
\hline \multicolumn{8}{|l|}{ REMARKS } \\
\hline \multicolumn{3}{|c|}{ the. $\alpha=0.069$} & & & & & \\
\hline \multicolumn{3}{|c|}{ Ane $B-0.218$} & & & & & \\
\hline & & & & & & & \\
\hline & & & & & & & \\
\hline
\end{tabular}




\section{COUNTER DATA SHEET}

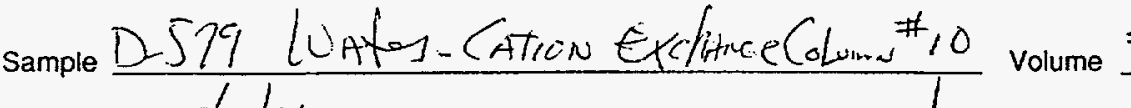
Sample Date $9 / 18 / 96$ _ 口PF-11 口PO-97 counted By

$$
\neq 46
$$

Date Counted $9-25-56$

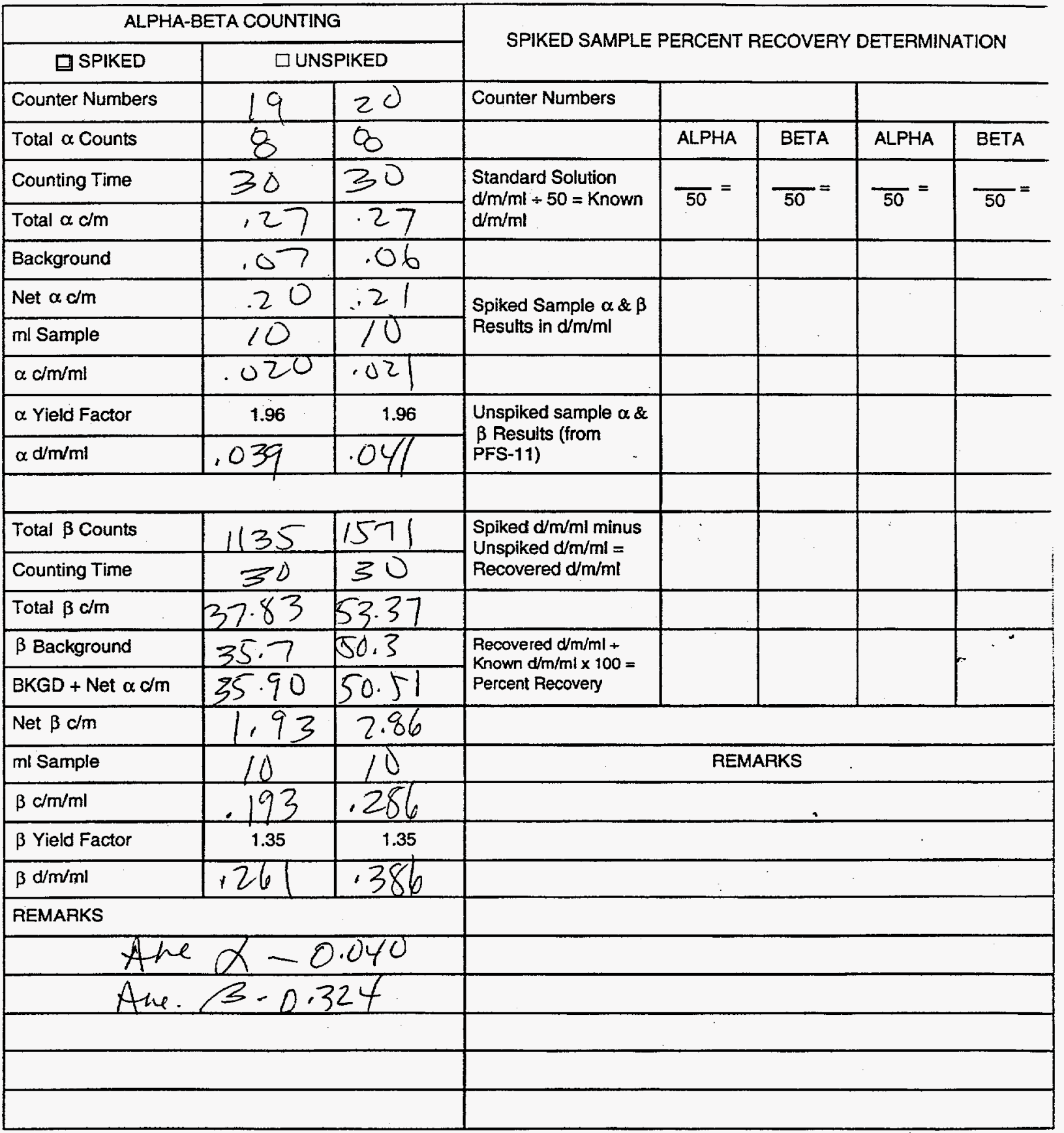


Date: $9 / 18 / 96$ Bldg: 579 CL. Sample: $\$ 48$

ype of Sample ch. Muied Bed Exchenge hppar Drawn Gallons: 63 . Requestor: Cheshte sh leex

ANALYSES DESIRED: $\quad \bar{X} \alpha, \bar{X} \beta .-\mathrm{jpH},=\mathrm{Hg}, \square$ Ammonia Nitrogen - Total Solids, _... Volume for $20 \%$ Bottoms

Other:

Date Analyzed: $9-2 T-96$ Results: $\alpha-0.039 \mathrm{~d} / \mathrm{m} / \mathrm{m} /$.

$$
3-0.486 \mathrm{~d} / \mathrm{m} / \mathrm{ml} \text {. }
$$

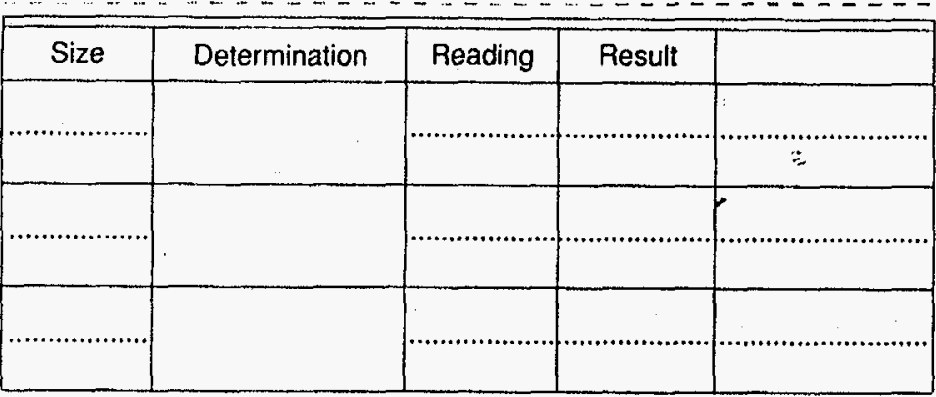

\begin{tabular}{|l|l|l|}
\hline & Total Solids & Total Solids \\
\hline Dish Number & & \\
\hline Wt. Dish + Wet Sample & & \\
\hline Wt. Dish & & \\
\hline Wt. Sample & & \\
\hline Wt. Dish + Dry Sample & & \\
\hline Wt. Dish & & \\
\hline Wt. Dry Sample & & \\
\hline Remarks and Calculations: & & \\
\end{tabular}

REQUEST FOR ANALYSIS

6471

Date: $9 / 18 / 96$ Bldg: 579 CL. Sample: 447 Type of Sample:Watew - Mifed Bul Exchenge Chumen - II Gallons: 63 Requestor: Chulath thelaex

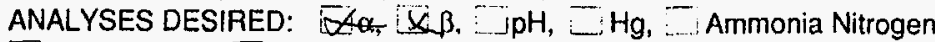
$\square$ Total Solids, - volume for $20 \%$ Bottoms

Other:

Date Analyzed: $9-25-96$ Analyst: Dein

Results: of $-0.044 \mathrm{~d} / \mathrm{m} / \mathrm{m} /$. $3-0.871 \mathrm{~d} / \mathrm{m} / \mathrm{m} /$.

\begin{tabular}{|c|c|c|c|c|}
\hline Size & Determination & Reading & Result & \\
\hline & & & & \\
\hline & & & & \\
\hline$\ldots$ & & & & \\
\hline & & & & $\therefore$ \\
\hline & & ' & & $z$ \\
\hline
\end{tabular}

\begin{tabular}{|l|c|c|}
\hline & Total Solids & Total Solids \\
\hline Dish Number & & \\
\hline Wt. Dish + Wet Sample & & \\
\hline Wt. Dish & & \\
\hline Wt. Sample & & \\
\hline Wt. Dish + Dry Sample & & \\
\hline Wt. Dish & & \\
\hline Wt. Dry Sample & & \\
\hline Remarks and Calculations: & & \\
\end{tabular}




\section{COUNTER DATA SHEET}

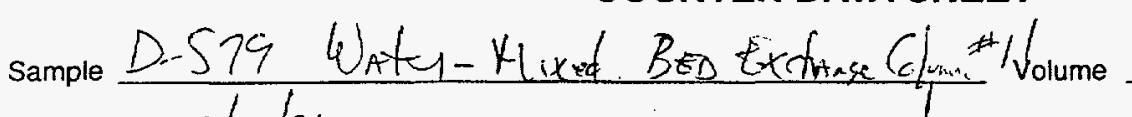
Sample Date $\frac{9 / 18 / 96}{\square P F S-11} \square$ PO-97 counted By

$\neq 47$

Date Counted $9 / 25 / 96$

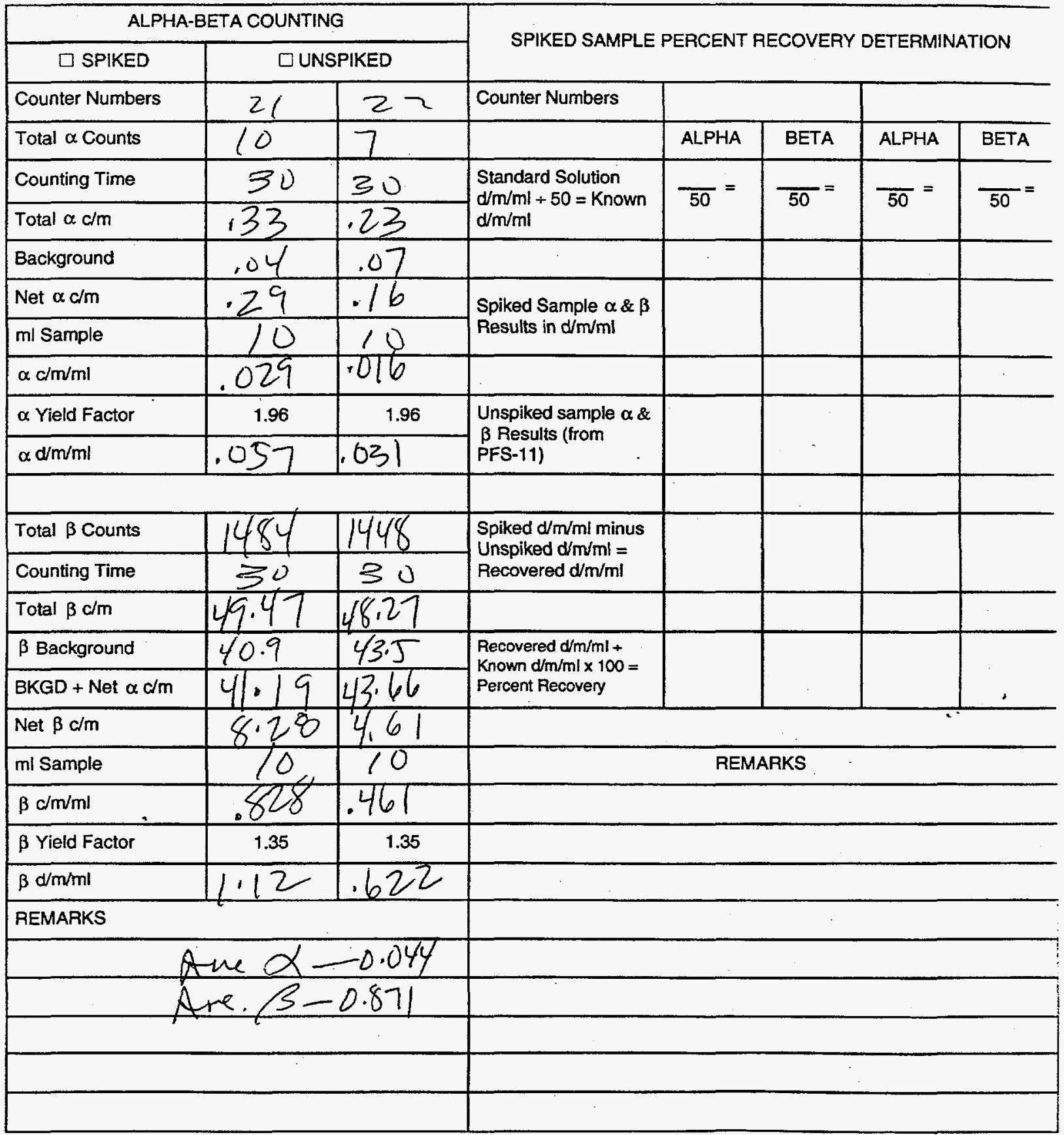




\section{COUNTER DATA SHEET}

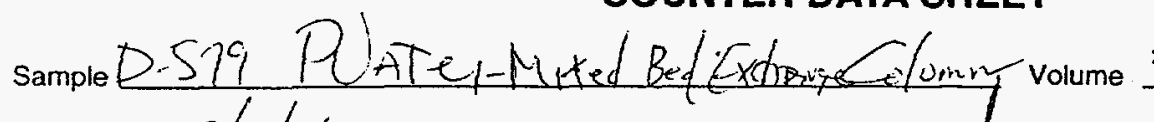
Sample Date $9 / 15 / 96$ DPFS-11 $\square$ PO-97 Counted By_t Date Counted $q / 25 / 76$

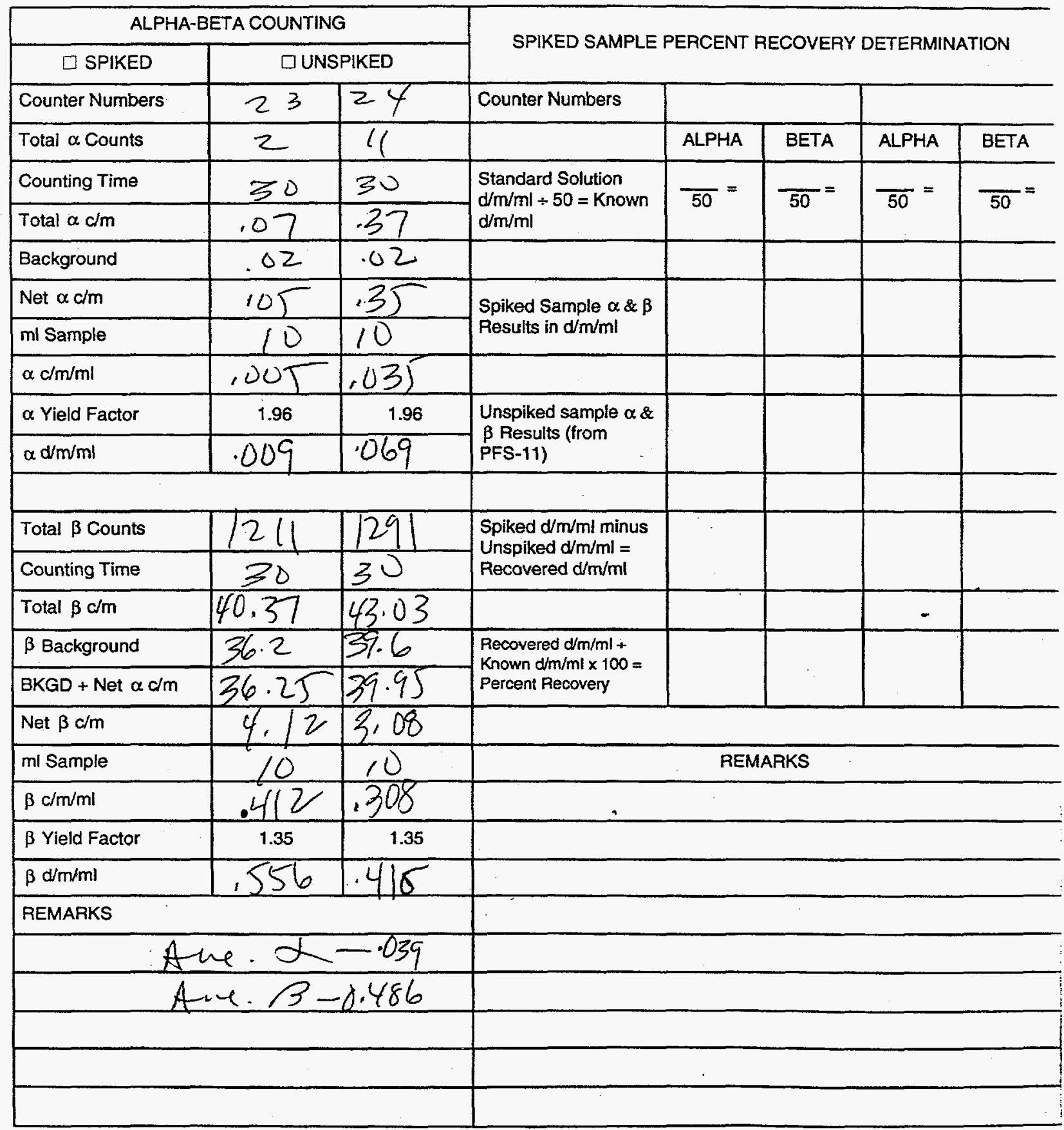


REQUEST FOR ANALYSIS

Date: $9 / 18 / 96$ Bldg: 579 CL. Sample: $260926-703$

Type of Sample: Watew - Queside thanher

Gallons: 63 .

Requestor: Chachate shalan

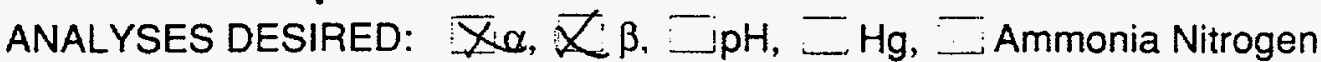
Z Total Solids, $=$ volume for $20 \%$ Bottoms

Other:

Date Analyzed:

Results:

Analyst: $M$

$$
\begin{aligned}
& \alpha=0.03 \mathrm{D} / \mathrm{m} / \mathrm{mL}, \\
& \beta=0.10 \mathrm{D} / \mathrm{m} / \mathrm{ml},
\end{aligned}
$$

\begin{tabular}{|c|c|c|c|c|}
\hline Size & Determination & Reading & Result & \\
\hline & & & & - \\
\hline & & & & \\
\hline & & & & \\
\hline & & & & \\
\hline & & & & \\
\hline & & & & \\
\hline
\end{tabular}

\begin{tabular}{|l|l|l|}
\hline & Total Solids & Total Solids \\
\hline Dish Number & & \\
\hline Wt. Dish + Wet Sample & & \\
\hline Wt. Dish & & \\
\hline Wt. Sample & & \\
\hline Wt. Dish + Dry Sample & & \\
\hline Wt. Dish & & \\
\hline Wt. Dry Sample & & \\
\hline Remarks and Calculations: & & \\
& & \\
\end{tabular}




\section{COUNTER DATA SHEET}

Sample RLD6 579 WACON- OUTSDO MNHOLE VOIUme 6 OZ Sample Date $9 / 18 / 46$ DPFS-11 QPO-97 Counted By DA Date Counted $9 / 26 / 96$

\begin{tabular}{|c|c|c|c|c|c|c|c|}
\hline \multicolumn{3}{|c|}{ ALPHA-BETA COUNTING } & \multirow{2}{*}{\multicolumn{5}{|c|}{ SPIKED SAMPLE PERCENT RECOVERY DETERMINATION }} \\
\hline \multirow{2}{*}{$\frac{\square \text { SPIKED }}{\text { Counter Numbers }}$} & \multicolumn{2}{|c|}{ ¿UNSPIKED } & & & & & \\
\hline & 27 & $2 B$ & \multirow[t]{2}{*}{ Counter Numbers } & \multirow[b]{2}{*}{ ALPHA } & \multirow[b]{2}{*}{ BETA } & \multirow[b]{2}{*}{ ALPHA } & \multirow[b]{2}{*}{ BETA } \\
\hline Total $\alpha$ Counts & 9 & 22 & & & & & \\
\hline Counting Time & 30 & 30 & \multirow{2}{*}{$\begin{array}{l}\text { Standard Solution } \\
d / m / m l+50=\text { Known } \\
d / m / m l\end{array}$} & \multirow{2}{*}{$\overline{50}=$} & \multirow{2}{*}{$\overline{50}=$} & \multirow{2}{*}{$\overline{50}=$} & \multirow{2}{*}{$\overline{50}=$} \\
\hline Total $\alpha \mathrm{c} / \mathrm{m}$ & 30 & .73 & & & & & \\
\hline Background & .09 & .34 & & & & & \\
\hline Net $\alpha \mathrm{c} / \mathrm{m}$ & .21 & .39 & \multirow{2}{*}{$\begin{array}{l}\text { Spiked Sample } \alpha \text { \& } \beta \\
\text { Results in } \alpha / m / m l\end{array}$} & & & & \\
\hline $\mathrm{ml}$ Sample & 20 & 20 & & & & & \\
\hline$\alpha \mathrm{c} / \mathrm{m} / \mathrm{ml}$ & .011 & .020 & & & & & \\
\hline$\alpha$ Yield Factor & 1.96 & 1.96 & \multirow{2}{*}{$\begin{array}{l}\text { Unspiked sample } \alpha \text { \& } \\
\beta \text { Results (from } \\
\text { PFS-11) }\end{array}$} & & & & \\
\hline$\alpha d / m / m l$ & .021 & .038 & & & & & \\
\hline & & & \multirow{3}{*}{$\begin{array}{l}\text { Spiked } d / \mathrm{m} / \mathrm{ml} \text { minus } \\
\text { Unspiked } d / m / m l= \\
\text { Recovered } d / \mathrm{m} / \mathrm{ml}\end{array}$} & & & & \\
\hline Total $\beta$ Counts & 1565 & 1554 & & & & & \\
\hline Counting Time & 30 & 30 & & & & & \\
\hline Total $\beta \mathrm{c} / \mathrm{m}$ & 52.2 & 51.8 & & & & & \\
\hline$\beta$ Background & 50.1 & 50.4 & \multirow{2}{*}{ 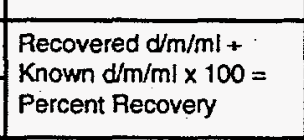 } & & & & \\
\hline BKGD + Net $\alpha \mathrm{c} / \mathrm{m}$ & 50.31 & 50.79 & & & & & \\
\hline Net $\beta c / m$ & 1.89 & 1.11 & \multirow{2}{*}{\multicolumn{5}{|c|}{ REMARKS }} \\
\hline mi Sample & 20 & 20 & & & & & \\
\hline$\beta \mathrm{c} / \mathrm{m} / \mathrm{ml}$ & .095 & .056 & \\
\hline$\beta$ Yield Factor & 1.35 & 1.35 & \\
\hline$\beta d / m / m l$ & .128 & .075 & \\
\hline \multicolumn{8}{|l|}{ REMARKS } \\
\hline \multicolumn{3}{|c|}{$\alpha=0.03 \mathrm{p} / \mathrm{m} / \mathrm{m}}$, & \\
\hline \multirow{2}{*}{\multicolumn{3}{|c|}{$\beta=0.10 \mathrm{D} / \mathrm{m} / \mathrm{M}_{\mathrm{.}}$}} & \\
\hline & & & \\
\hline \multicolumn{8}{|c|}{\begin{tabular}{|l|l|} 
& \\
\end{tabular}} \\
\hline
\end{tabular}




\section{REQUEST FOR ANALYSIS, $-9.649217-105$} Date: 4.34.4h Bldg: $57 \%$ CL. Sample: 157

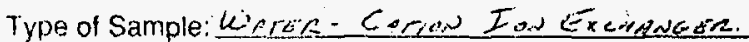
Gallong:s Dec: ise Requestor: Cingeoter SHoceren ANALYSES DESIRED: $\Varangle \alpha, \bar{X}, \beta, \ldots \mathrm{pH}, \ldots \mathrm{Hg}, \quad$ Ammonia Nitrogen Total Solids. .... Volume for $20 \%$ Bottoms

Other:

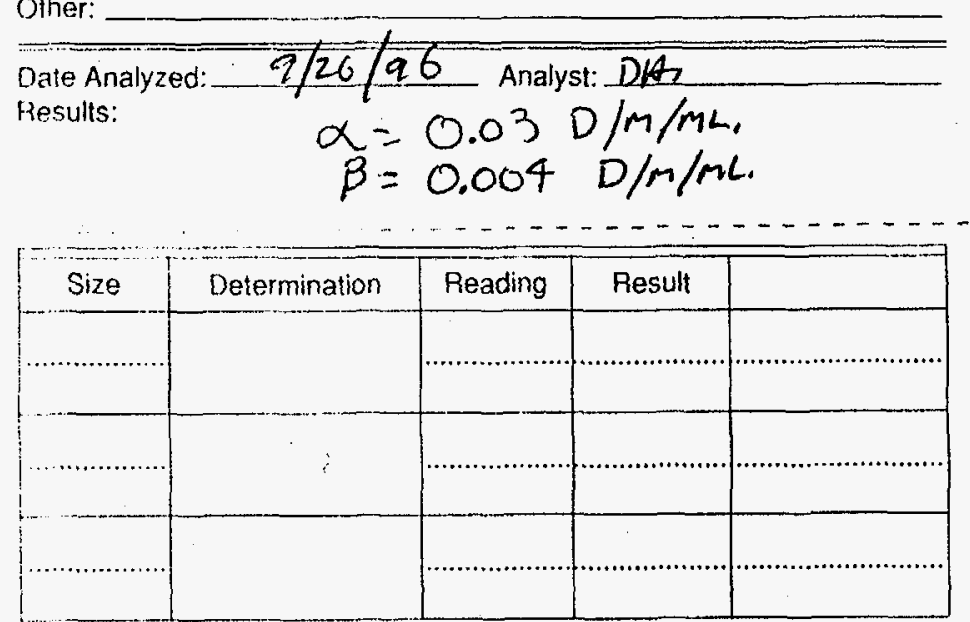

\begin{tabular}{|l|l|l|}
\hline & Total Solids & Total Solids \\
\hline Dish Number & & \\
\hline Wt. Dish + Wet Sample & & \\
\hline Wt. Dish & & \\
Wt. Sample & & \\
\hline Wt. Dish + Dry Sample & & \\
\hline Wt. Dish & & \\
Wt. Dry Sample & & \\
\hline Fiemarks and Calculations: & & \\
\hline
\end{tabular}

\section{- . - REQUEST FOR ANALYSIS}

$6: 14$

Date: $9-25$ Fic Bldg: 579 CL. Sample: $\quad 67$ Type of Sample: WarER - Manrec á CL-960926-104 Gattons: $60 \mathrm{~mL}$ Requestor: Cripetorte Shelezes

ANALYSES DESIRED: $\propto \alpha, X \beta, \cdots \mathrm{pH},-\mathrm{Hg}, \quad$ Ammonia Nitrogen Total Solids, Volume for $20 \%$ Bottoms

Other:

Date Analyzed: $\quad 9 / 26 / 96$ Analyst: $1 / 4$ Results:

$$
\begin{aligned}
& \alpha=0.37 \mathrm{P} / \mathrm{m} / \mathrm{mL} . \\
& \beta=0.99 \mathrm{D} / \mathrm{m} / \mathrm{mL} .
\end{aligned}
$$

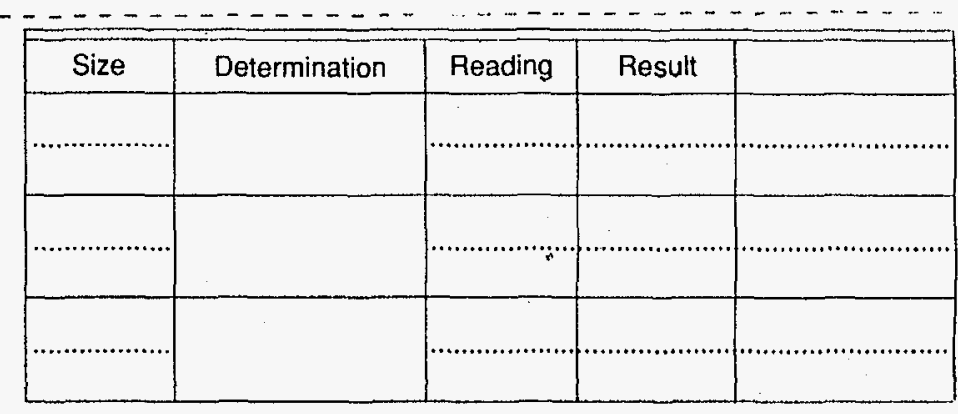

\begin{tabular}{|l|c|c|}
\hline & Total Solids & Total Solids \\
\hline Dish Number & & \\
\hline Wt. Dish + Wet Sample & & \\
\hline Wt. Dish & & \\
\hline Wt. Sample & & \\
\hline Wt. Dish + Dry Sample & & \\
\hline Wt. Dish & & \\
\hline Wt. Dry Sample & & \\
\hline Remarks and Calculations: & \\
\hline
\end{tabular}




\section{COUNTER DATA SHEET}

Sample BLO6 529 WAZE2-CATLON ExCHANGTR Volume

CL-960926-105

simple Date $9 / 24 / q b$ GPFS-11 $\square$ PO-97 counted By_Mk

Date Counted $\$ / 26 / q 6$

\begin{tabular}{|c|c|c|c|c|c|c|c|}
\hline \multicolumn{3}{|c|}{ ALPHA-BETA COUNTING } & \multirow{2}{*}{\multicolumn{5}{|c|}{ SPIKED SAMPLE PERCENT RECOVERY DETERMINATION }} \\
\hline \multirow{2}{*}{$\frac{\text { ¿ SPIKED }}{\text { Counter Numbers }}$} & \multicolumn{2}{|c|}{ UNSPIKED } & & & & & \\
\hline & 22 & 28 & \multirow[t]{2}{*}{ Counter Numbers } & \multirow[b]{2}{*}{ ALPHA } & \multirow[b]{2}{*}{ BETA } & \multirow[b]{2}{*}{ ALPHA } & \multirow{2}{*}{ BETA } \\
\hline Total $\alpha$ Counts & 7 & 27 & & & & & \\
\hline Counting Time & 20 & 30 & \multirow{2}{*}{$\begin{array}{l}\text { Standard Solution } \\
\mathrm{d} / \mathrm{m} / \mathrm{ml}+50=\text { Known } \\
\mathrm{d} / \mathrm{m} / \mathrm{ml}\end{array}$} & \multirow{2}{*}{$\overline{50}=$} & \multirow{2}{*}{$\overline{50}=$} & \multirow{2}{*}{$\overline{50}=$} & \multirow{2}{*}{$\overline{50}=$} \\
\hline Total $\alpha \mathrm{c} / \mathrm{m}$ & 23 & .90 & & & & & \\
\hline Background & .09 & .34 & & & & & \\
\hline Net $\alpha \mathrm{c} / \mathrm{m}$ & .14 & .56 & \multirow{2}{*}{$\begin{array}{l}\text { Spiked Sample } \alpha \& \beta \\
\text { Results in } d / \mathrm{m} / \mathrm{ml}\end{array}$} & & & & \multirow{2}{*}{$\cdot$} \\
\hline ml Sample & 20 & 20 & & & & & \\
\hline$\alpha \mathrm{c} / \mathrm{m} / \mathrm{ml}$ & .007 & .028 & & & & & \\
\hline$\alpha$ Yield Factor & 1.96 & 1.96 & \multirow{2}{*}{$\begin{array}{l}\text { Unspiked sample } \alpha \& \\
\beta \text { Results (from } \\
\text { PFS-11) }\end{array}$} & & & & \\
\hline$\alpha \mathrm{d} / \mathrm{m} / \mathrm{ml}$ & .014 & .054 & & & & & \\
\hline Total $\beta$ Counts & 1505 & 1533 & \multirow{2}{*}{$\begin{array}{l}\text { Spiked d/m/ml minus } \\
\text { Unspiked } \mathrm{d} / \mathrm{m} / \mathrm{ml}= \\
\text { Recovered } \mathrm{d} / \mathrm{m} / \mathrm{ml}\end{array}$} & & & & \\
\hline Counting Time & 30 & 30 & & & & & \\
\hline Total $\beta \mathrm{c} / \mathrm{m}$ & 50.2 & 51.1 & & & & & \\
\hline$\beta$ Background & 50.1 & 504 & \multirow{2}{*}{$\begin{array}{l}\text { Recovered } \mathrm{d} / \mathrm{m} / \mathrm{ml}+ \\
\text { Known } \mathrm{d} / \mathrm{m} / \mathrm{m} / \times 100= \\
\text { Percent Recovery }\end{array}$} & & & & \\
\hline $\mathrm{BKGD}+\mathrm{Net} \alpha \mathrm{cm}$ & 50.24 & 50.96 & & & & & \\
\hline Net $\beta c / m$ & & .14 & & & & & \\
\hline ml Sample & 20 & 20 & \multicolumn{5}{|c|}{ REMARKS } \\
\hline$\beta \mathrm{c} / \mathrm{m} / \mathrm{ml}$ & & .007 & \\
\hline$\beta$ Yield Factor & 1.35 & 1.35 & \\
\hline$\beta \mathrm{d} / \mathrm{m} / \mathrm{ml}$ & - & 6009 & \\
\hline \multicolumn{8}{|l|}{ REMARKS } \\
\hline \multicolumn{3}{|c|}{$\alpha=0.03 \mathrm{D} / \mathrm{m} / \mathrm{m}$} & \\
\hline \multicolumn{3}{|c|}{$\beta=0.004 \mathrm{D} / \mathrm{m} / \mathrm{mL}$} & \\
\hline \multicolumn{3}{|c|}{1} & & & & & \\
\hline 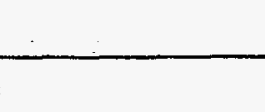 & & 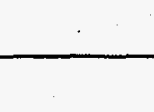 & \multicolumn{5}{|c|}{${ }_{2}$} \\
\hline
\end{tabular}




\section{COUNTER DATA SHEET}

Sample BLD6 579 WAER - MANAOWO 61 volume $60 \mathrm{~mL}$.

sampie Date $q / 25 / 96$ DPFS-11 D PO-97 Counted By_mir Date Counted $9 / 26 / 26$

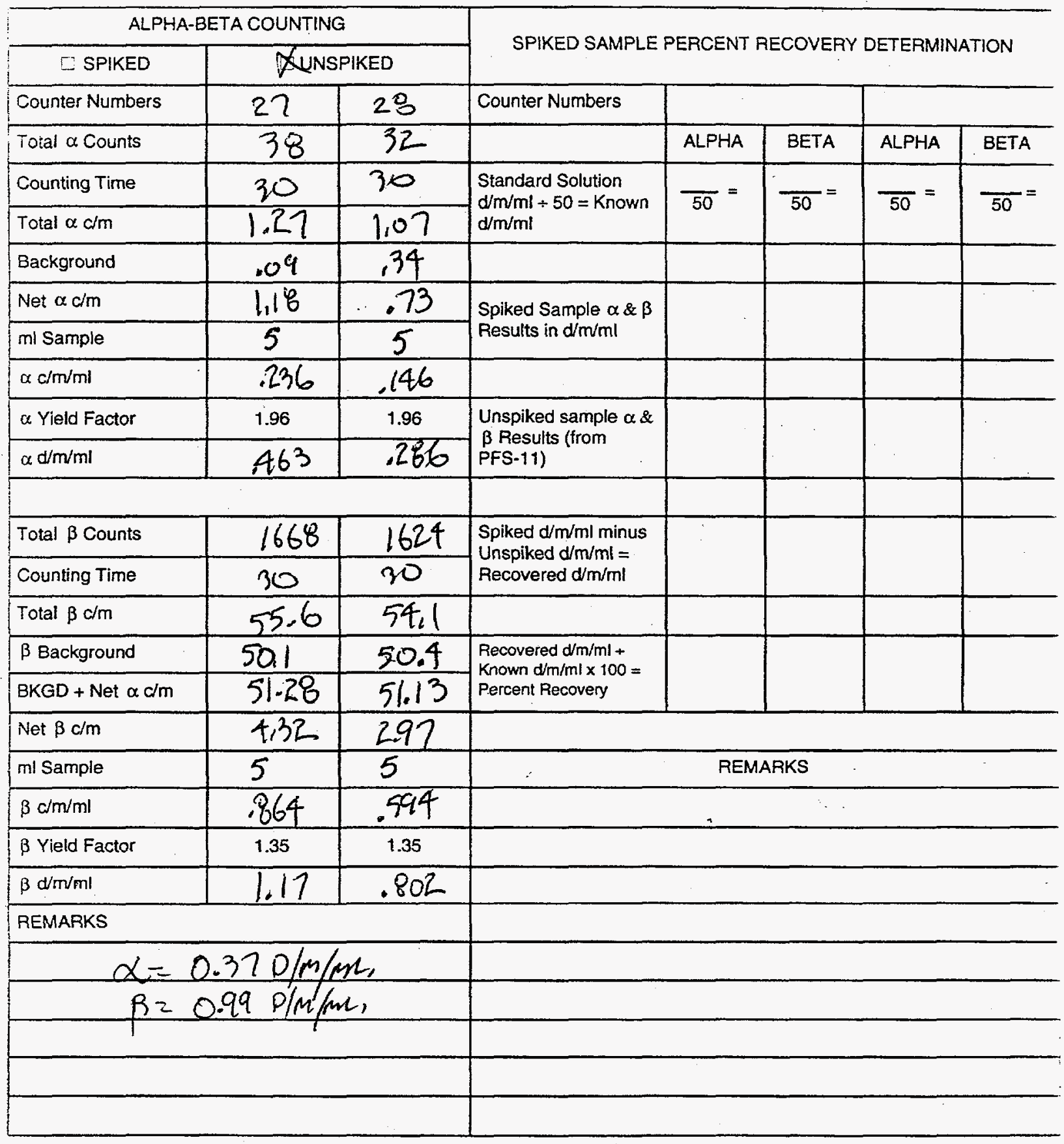


ANALYTICAL CHEMISTRY LABORATORY

Argonne National Laboratory

Argonne, IL 60439

REPORT OF ANALYTICAL RESULTS

\section{Sample Material: Water}

Date Received: $\quad$ 9/20/96

Submitted by:

Charlotte Sholeen, ESH-HP, 306

Date Reported: $\quad$ 10/7/96

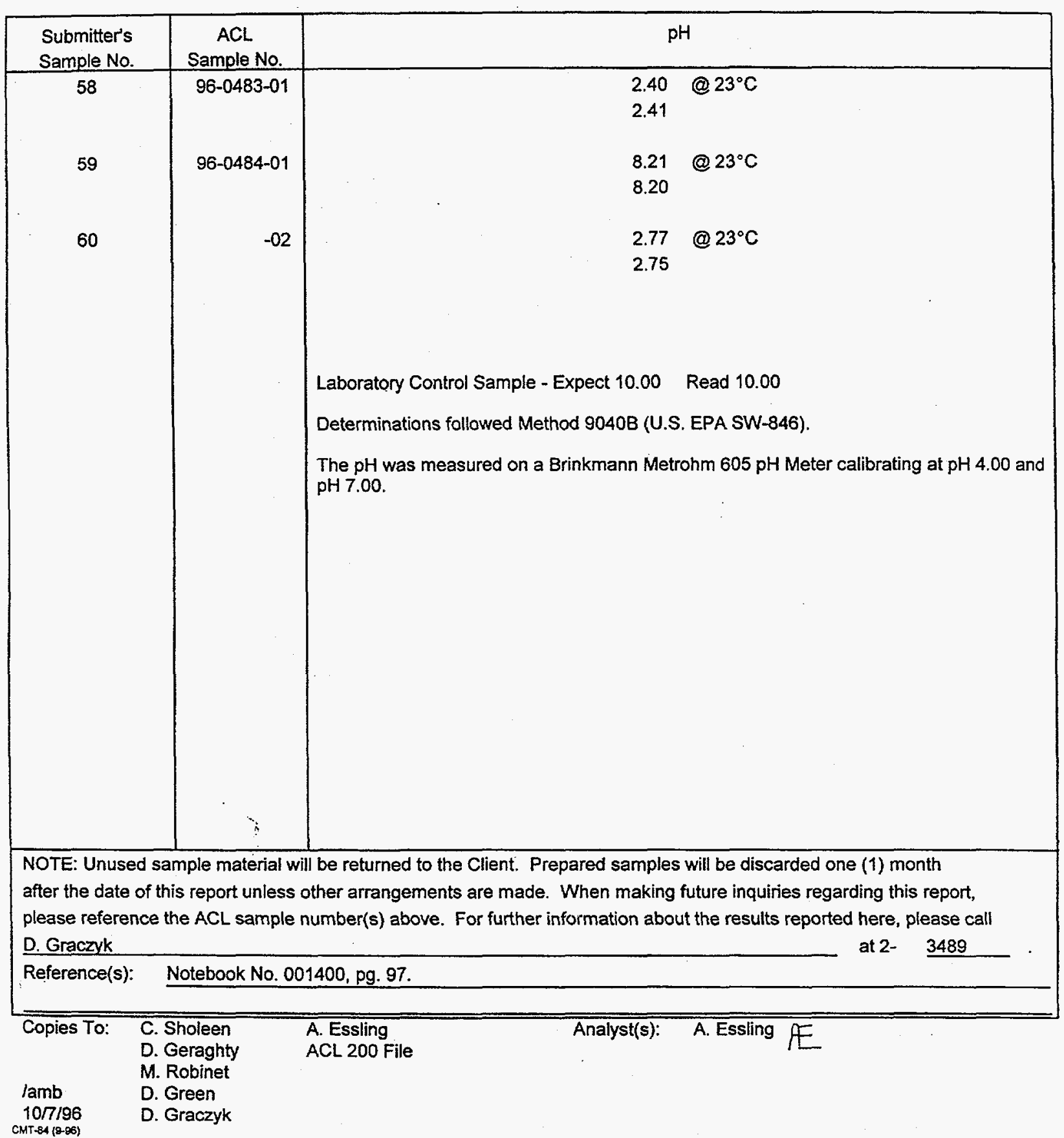


ANALYTICAL CHEMISTAY LABORATORY

Argonne National Laboratory

Argonne, IL 60439

REPORT OF ANALYTICAL RESULTS

Sample Material: Resin

Submitted by:

Charlotte Sholeen, ESH-HP, 202

Date Received:

$9 / 20 / 96$

Date Reported: $\quad$ 10/16/96

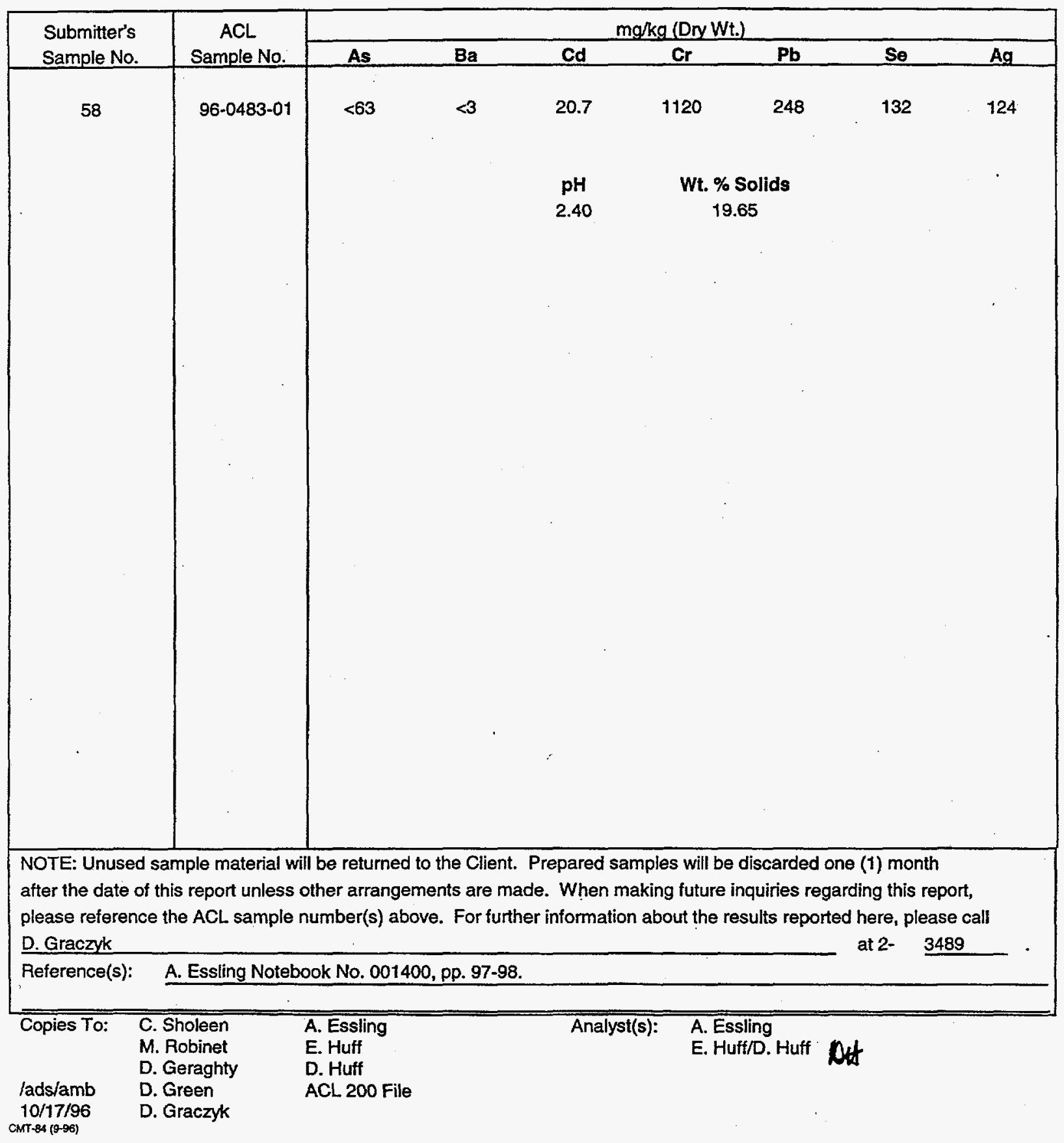


ANALYTICAL CHEMISTRY LABORATORY

Argonne National Laboratory

Argonne, IL 60439

\title{
ICP-AES Analysis Report
}

\author{
Spec. No.: $58548 \mathrm{EX}$
}

Sample Material: TCLP Extract of Resin

Submitted by: Charlotte Sholeen ESH/HP306

Date Received:09-20-96

Reporting Units: ug/mL

Date Reported:02-25-97

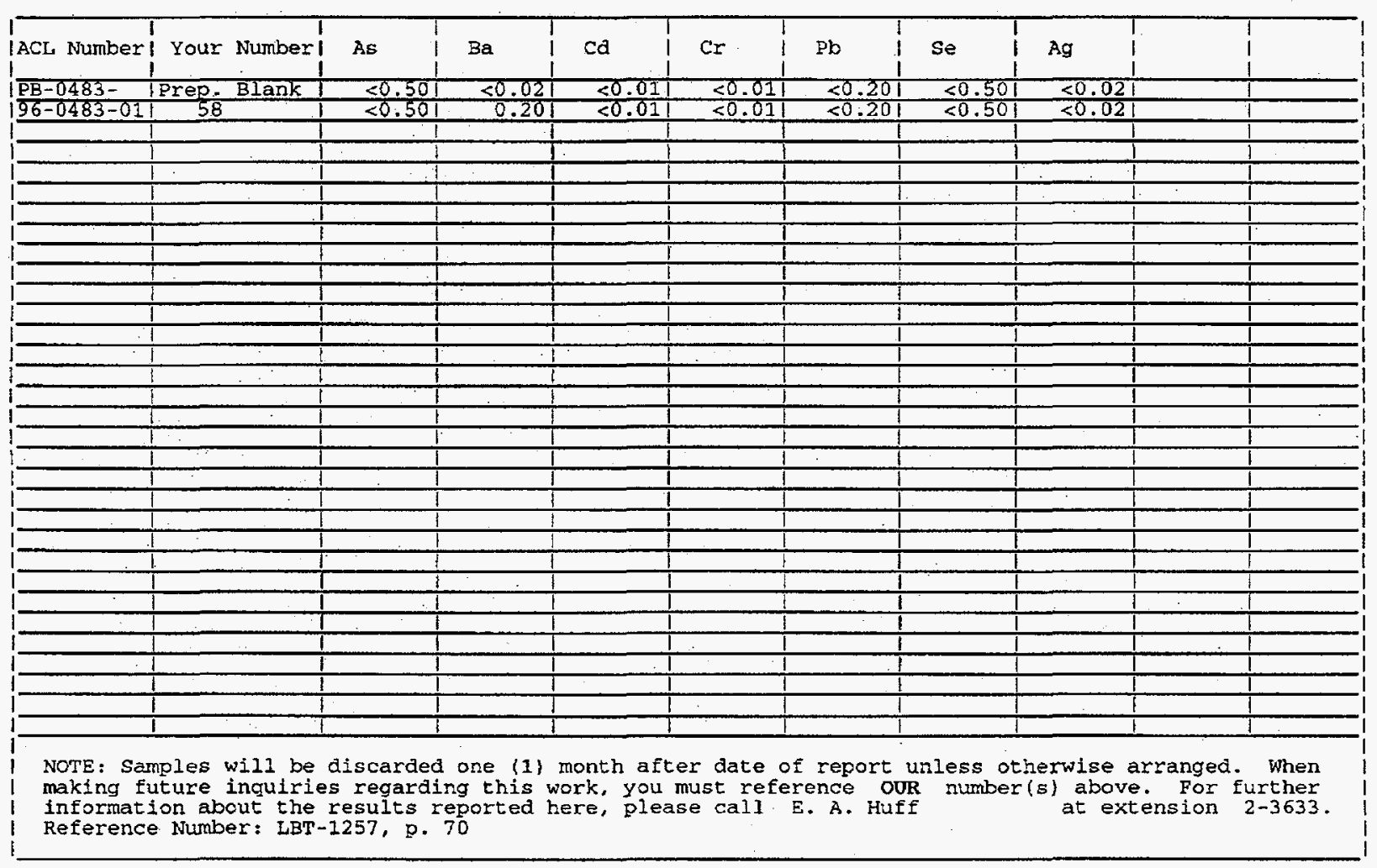

Estimated Accuracy:+1-208
Copies To: Co Sholeen "I. Tenkate
M. Robinet ACL Office
Analyst (s): E. Huff
D. Huff
L. Tenkate
D. Geraghty
D. Graczyk
D. Green

Remarks : 
ANALYTICAL CHEMISTRY LABORATORY

Argonne National Laboratory

Argonne, IL 60439

REPORT OF ANALYTICAL RESULTS

Sample Material: Water

Submitted by:

Charlotte Sholeen, ESH-HP, 202

Date Received:

9/20/96

Date Reported: $\quad$ 10/16/96

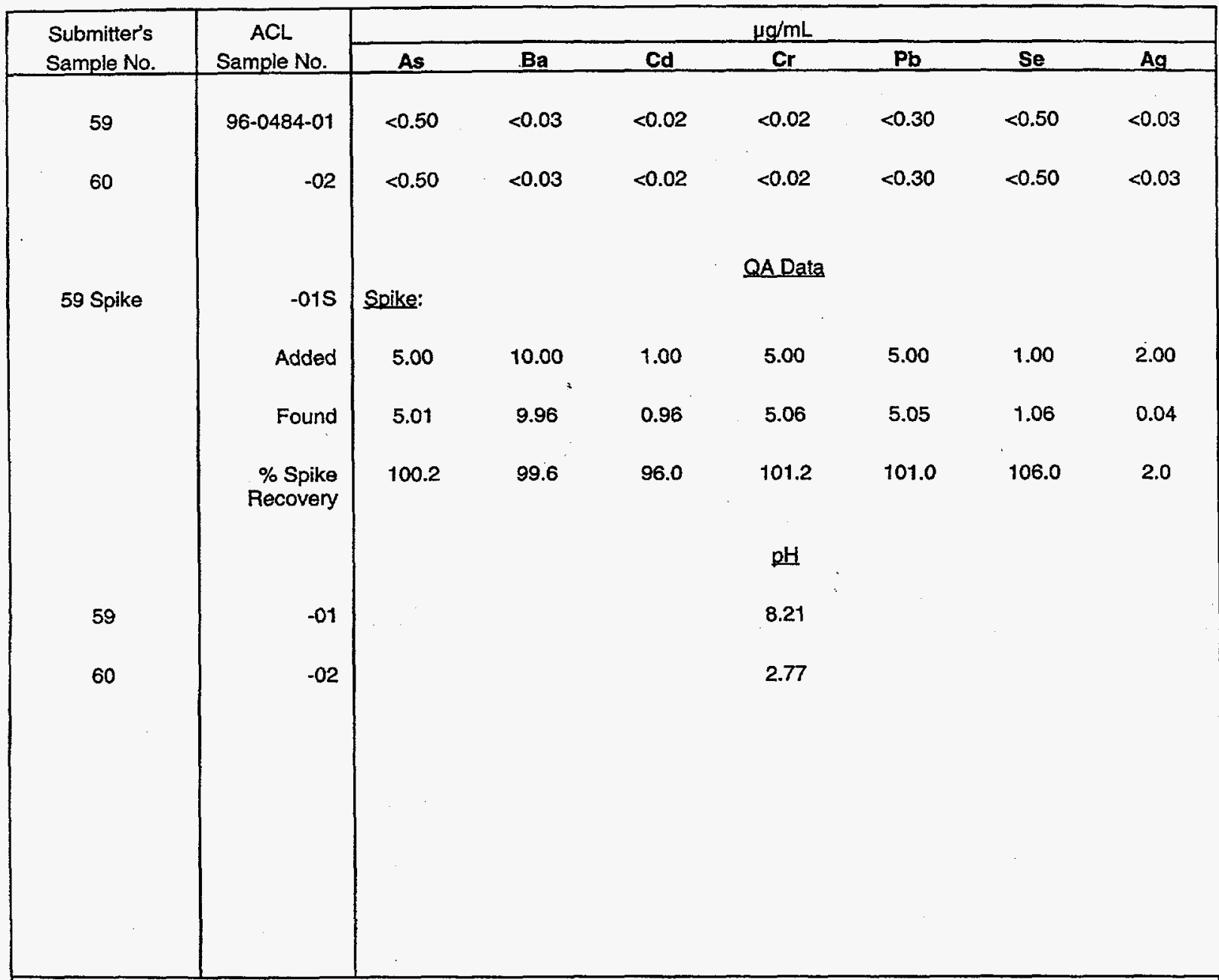

NOTE: Unused sample material will be returned to the Client. Prepared samples will be discarded one (1) month after the date of this report unless other arrangements are made. When making future inquiries regarding this report, please reference the ACL sample number(s) above. For further information about the results reported here, please call D. Graczyk

Reference(s): A. Essling Notebook No. 001400, pp. 97-98. at 2- 3489

\begin{tabular}{lllll}
\hline Copies To: & C. Sholeen & A. Essling & Analyst(s): & A. Essling \\
& M. Robinet & E. Huff & & E. Huff \\
& D. Geraghty & D. Huff & & D. Huff W/A \\
/ads/amb & D. Green & ACL 200 File & & \\
$10 / 17 / 96$ & D. Graczyk & & & \\
amr $: 49-96)$ & & & &
\end{tabular}


ANALYTICAL CHEMISTRY LABORATORY

Argonne National Laboratory

Argonne, IL 60439

REPORT OF ANALYTICAL RESULTS

Sample Material: $\quad$ Building 579 Resin

Date Received:

$9 / 20 / 96$

Submitted by:

C. Sholeen/D. Geraghty

Date Reported:

9/25/96

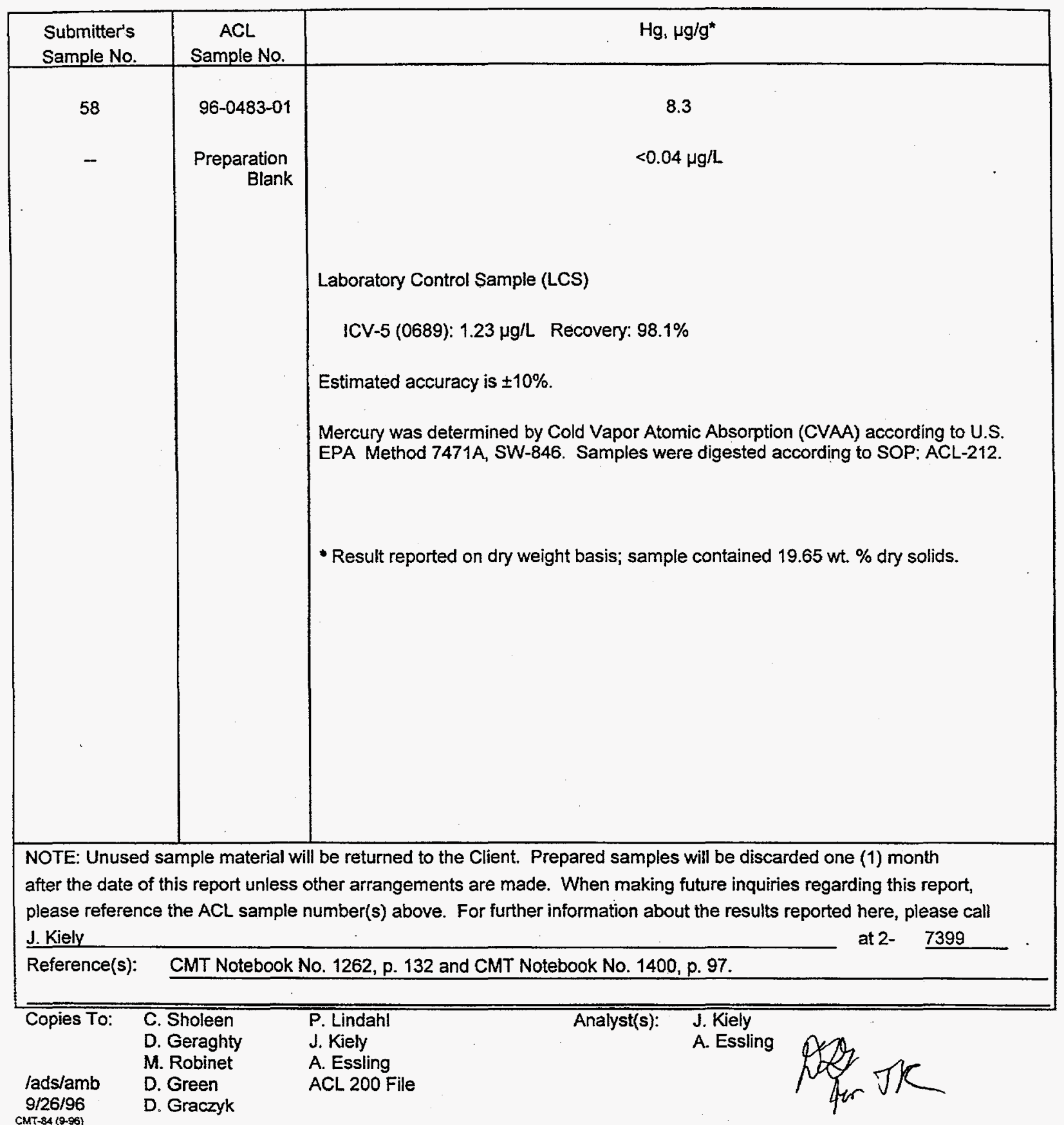


ANALYTICAL CHEMISTRY LABORATORY

Argonne National Laboratory

Argonne, IL 60439

REPORT OF ANALYTICAL RESULTS

Sample Material: $\quad$ TCLP Extract of Resin from Bidg. 579

Date Received:

$12 / 9 / 96$

Submitted by:

Charlotte Sholeen, ESH-HP, 202

Date Reported:

2/18/97

\begin{tabular}{|c|c|c|}
\hline $\begin{array}{l}\text { Submitter's } \\
\text { Sample No. }\end{array}$ & $\begin{array}{c}\text { ACL } \\
\text { Sample No. }\end{array}$ & $\mathrm{Hg}, \mu g / L$ \\
\hline 58 & $\begin{array}{r}96-0483-01 \\
\text { Blank } \\
\text { Extraction } \\
\text { Fluid \#1 }\end{array}$ & 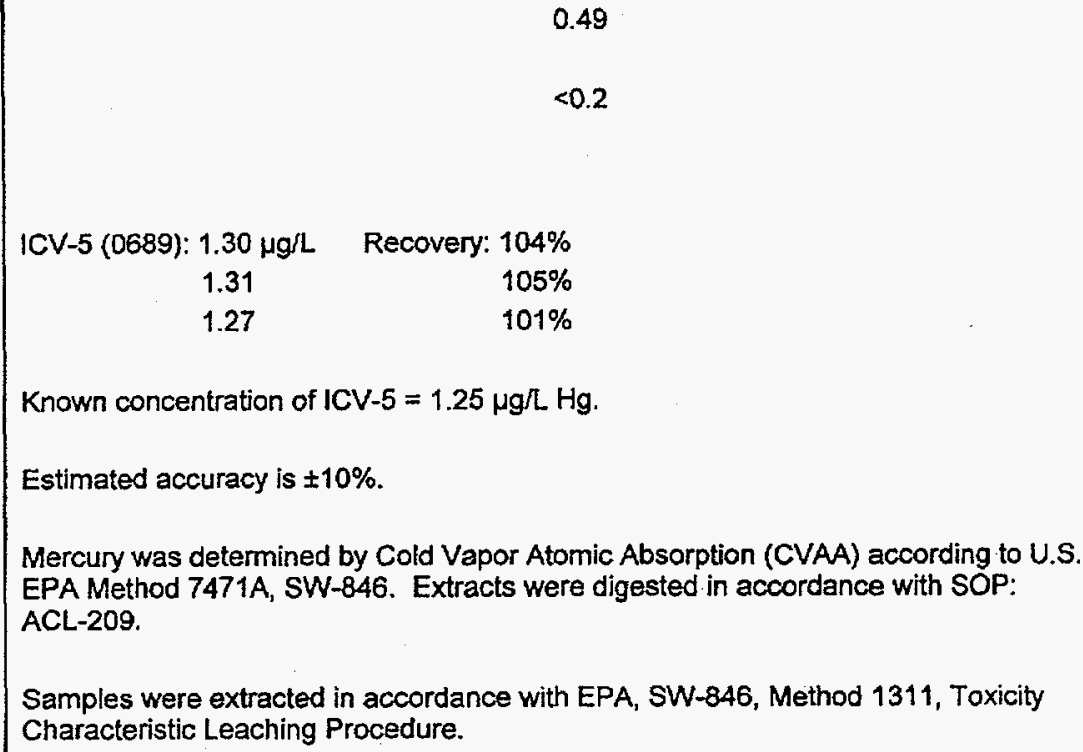 \\
\hline \multirow{2}{*}{\multicolumn{3}{|c|}{$\begin{array}{l}\text { NOTE: Unused sample material will be retumed to the Client. Prepared samples will be discarded one (1) month } \\
\text { after the date of this report unless other arrangements are made. When making future inquiries regarding this report, } \\
\text { please reference the ACL sample number(s) above. For further information about the results reported here, please call } \\
\text { L. TenKate } \\
\text { Reference(s): CMT Notebook No. } 1262, \mathrm{pg} .139 .\end{array}$}} \\
\hline & & \\
\hline Reference(s): & TCLP Extraction & - CMT Notebook No. 1143, pp. 141-143. \\
\hline $\begin{array}{l}\text { Copies To: } \\
\text { /ads } \\
2 / 18 / 97 \\
\text { MT }-84 \text { (8-96) }\end{array}$ & $\begin{array}{l}\text { C. Sholeen } \\
\text { D. Geraghty } \\
\text { M. Robinet } \\
\text { D. Green } \\
\text { D. Graczyk }\end{array}$ & 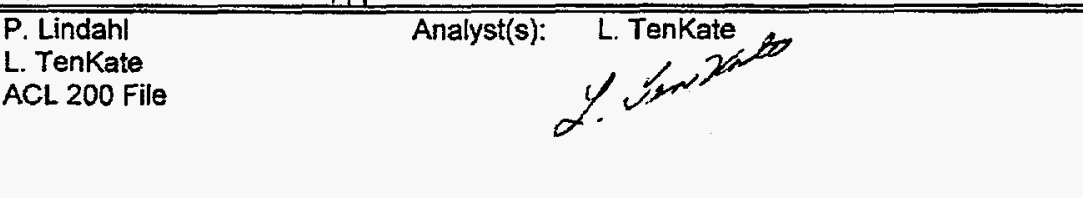 \\
\hline
\end{tabular}


ANALYTICAL CHEMISTRY LABORATORY

Argonne National Laboratory

Argonne, IL 60439

REPORT OF ANALYTICAL RESULTS

$\begin{array}{llll}\text { Sample Material: } & \text { Building } 579 \text { Water } & \text { Date Received: } & 9 / 20 / 96 \\ \text { Submitted by: } & \text { C. Sholeen/D. Geraghty } & \text { Date Reported: } & 9 / 25 / 96\end{array}$

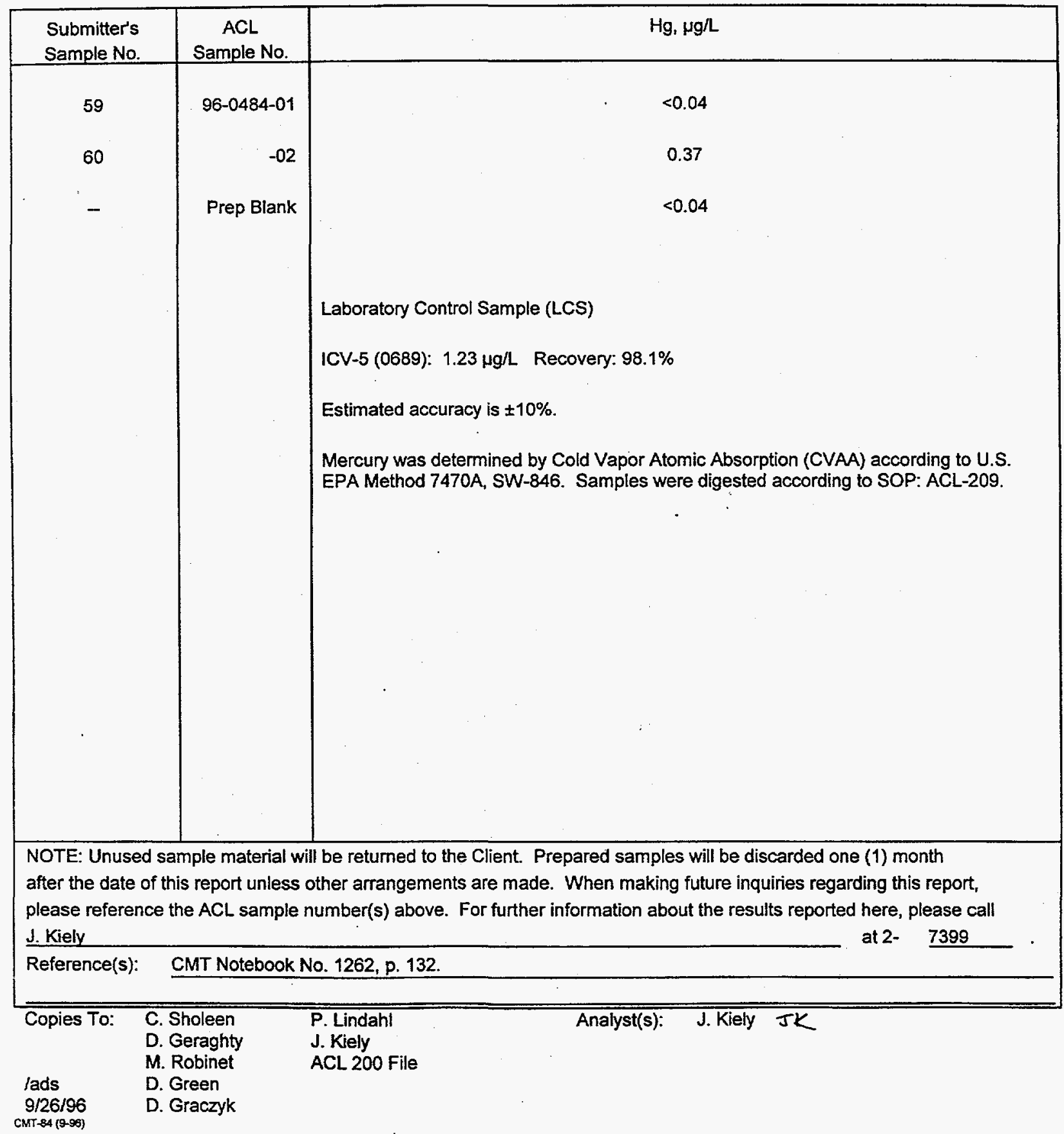


ANALYTICAL CHEMISTRY LABORATORY

Argonne National Laboratory

Argonne, IL 60439

REPORT OF ANALYTICAL RESULTS

Revised 12/20/96

Sample Material: $\quad$ Resin, Column in Bldg. 579

Submitted by:

Charlotte Sholeen, ESH-HP, 202

$\begin{array}{ll}\text { Date Received: } & 12 / 9 / 96 \\ \text { Date Reported: } & 12 / 18 / 96\end{array}$

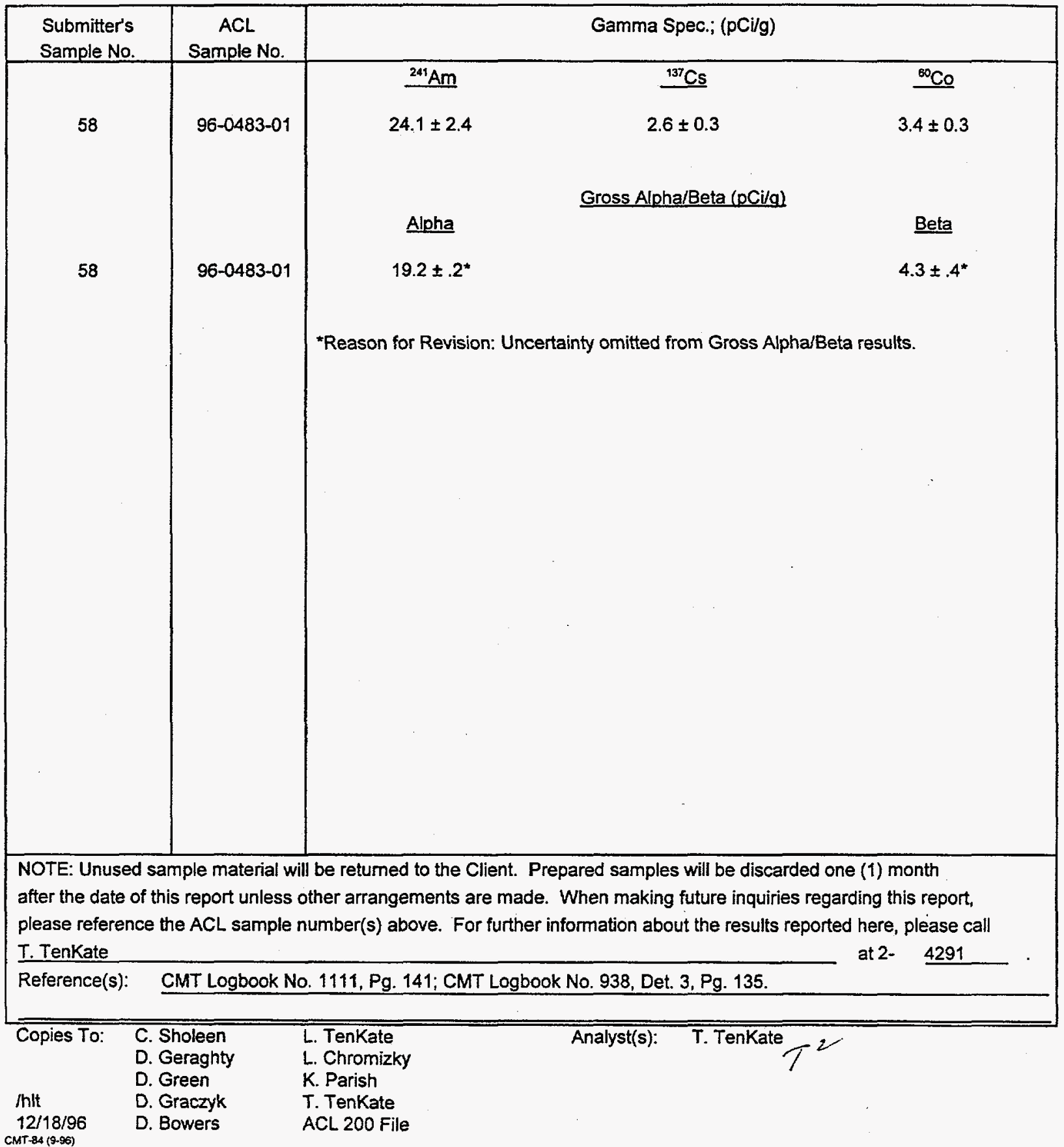


ANALYTICAL CHEMISTRY LABORATO

Argonne National Laboratory

Argonne, IL 60439

REPORT OF ANALYTICAL RESUI ?

Sample Material: $\quad$ Water, "579"

Submitted by:

Charlotte Sholeen, ESH-HP, 202

$\begin{array}{ll}\text { Date Received: } & 9 / 30 / 96 \\ \text { Date Reported: } & 10 / 29 / 96\end{array}$

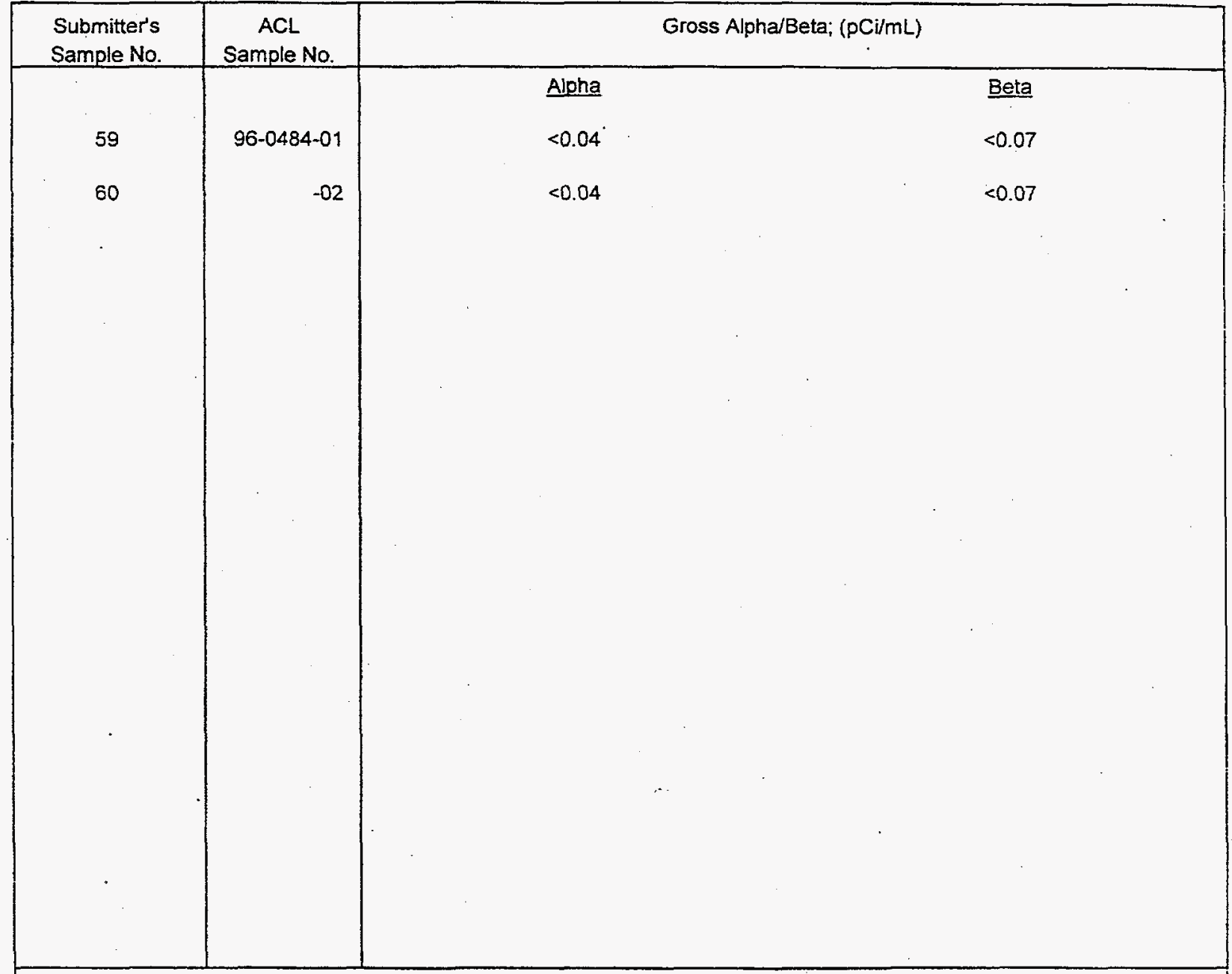

NOTE: Unused sample material will be returned to the Client. Prepared samples will be discarded one (1) month after the date of this report unless other arrangements are made. When making future inquiries regarding this report, please reference the $\mathrm{ACL}$ sample number(s) above. For further information about the results reported here, please call T. Tenkate at 2- 4291

Reference(s): CMT Logbook No. 1111, Pg. 126.

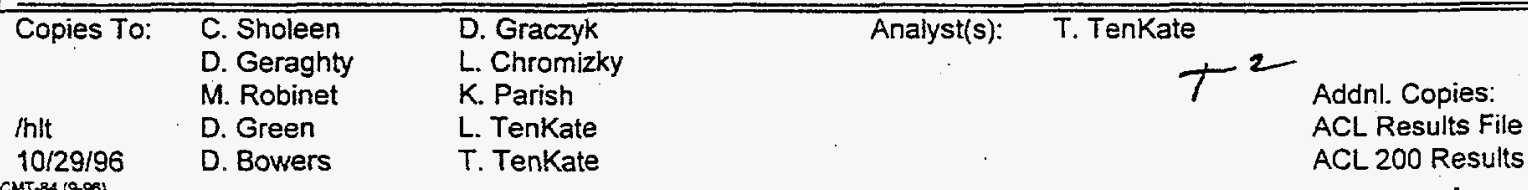




\section{ANALYTICAL CHEMISTRY LABORATORY \\ Argonne National Laboratory \\ Argonne, IL 60439}

\section{REPORT OF ANALYTICAL RESULTS}

$\begin{array}{llll}\text { Sample Material: } & \text { Water, "579" } & \text { Date Received: } & \text { 9/30/96 } \\ \text { Submitted by: } & \text { Charlotte Sholeen, ESH-HP, } 202 & \text { Date Reported: } & 10 / 2 / 96\end{array}$

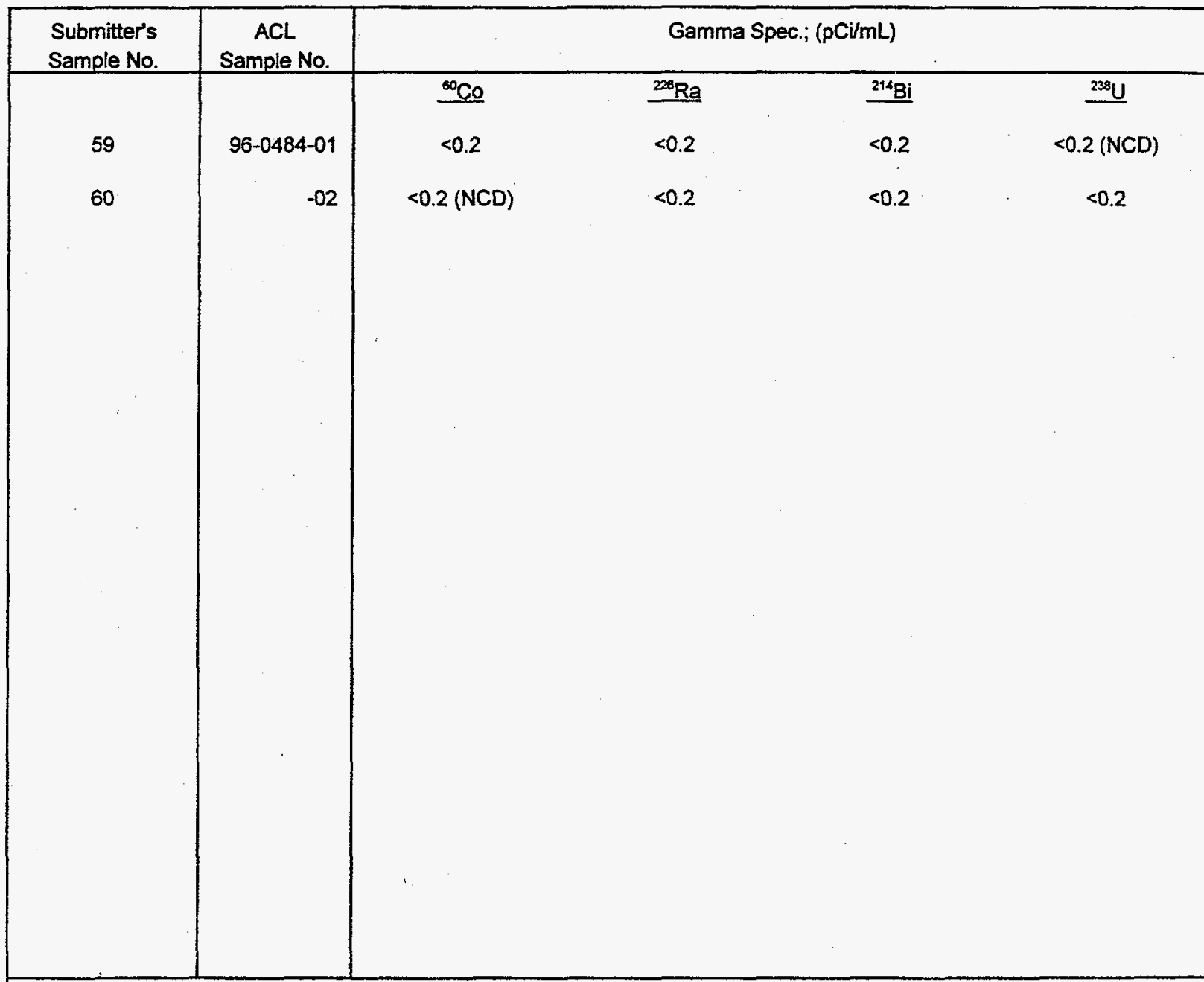

NOTE: Unused sample material will be returned to the Client. Prepared samples will be discarded one (1) month after the date of this report unless other arrangements are made. When making future inquiries regarding this report, please reference the ACL sample number(s) above. For further information about the results reported here, please call T. TenKate

Reference(s): CMT Logbook No. 1111, Pg. 126; CMT Logbook No. 1112, Det. 1, 9, and 10, Pg. 91; CMT Logbook No. 938, Det. 1, 3, and 6, Pg. 129.

\begin{tabular}{|c|c|c|c|c|}
\hline Copies To: & $\begin{array}{l}\text { C. Sholeen } \\
\text { D. Geraghty } \\
\text { M. Robinet } \\
\text { D. Green }\end{array}$ & $\begin{array}{l}\text { D. Graczyk } \\
\text { L. Chromizky } \\
\text { K. Parish } \\
\text { L. TenKate }\end{array}$ & Analyst(s): $\quad$ T. TenKate & $\begin{array}{l}\text { Addnl. Copies: } \\
\text { ACL } 200 \text { File }\end{array}$ \\
\hline
\end{tabular}

$10 / 2 / 96$

D. Bowers T. TenKate 
ANALYTICAL CHEMISTRY LABORATORY

Argonne National Laboratory

Argonne, IL 60439

REPORT OF ANALYTICAL RESULTS

$\begin{array}{llll}\text { Sample Material: } & \text { Resin } & \text { Date Received: } & 9 / 20 / 96 \\ \text { Submitted by: } & \text { Charlotte Sholeen, ESH-HP, } 202 & \text { Date Reported: } & 12 / 11 / 96\end{array}$

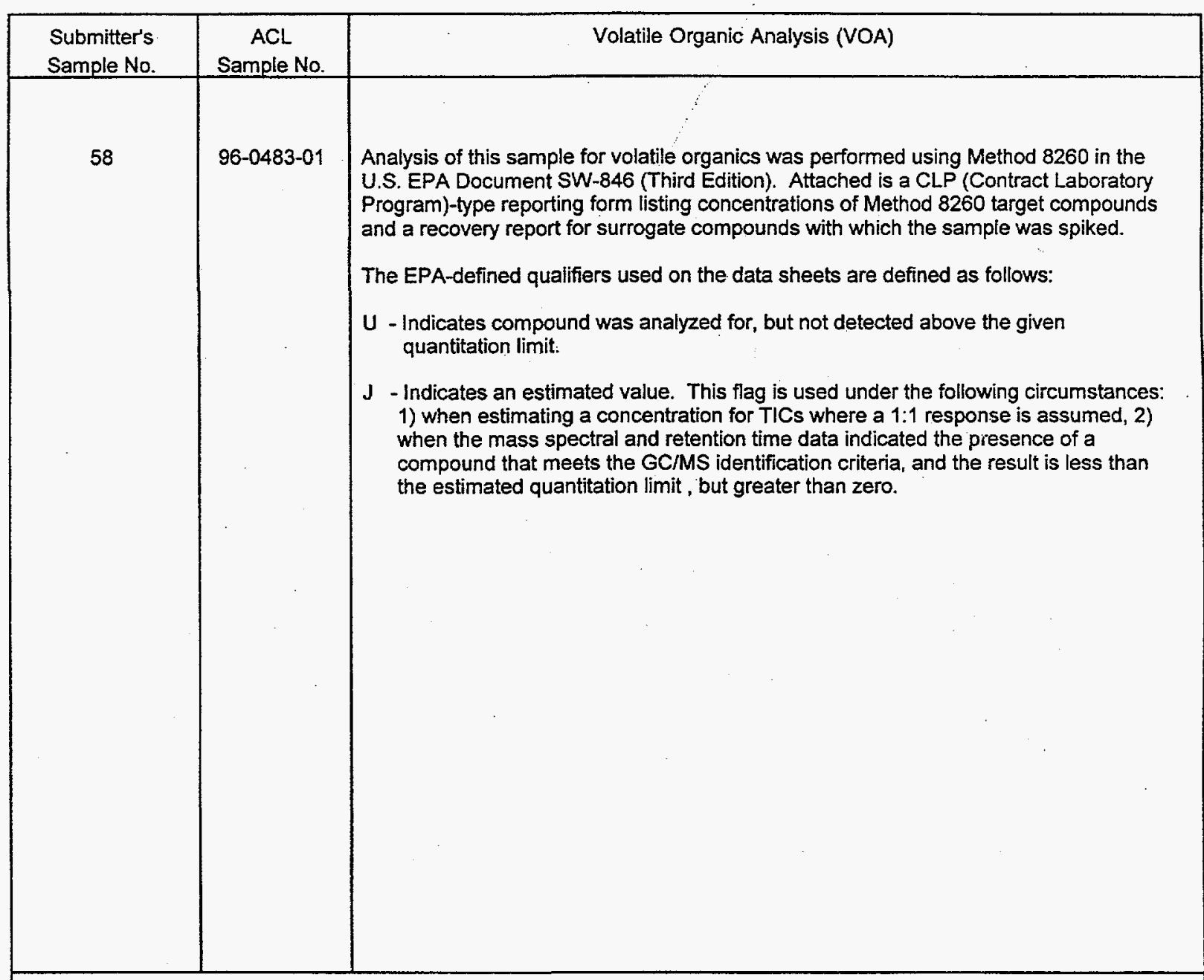

NOTE: Unused sample material will be returned to the Client. Prepared samples will be discarded one (1) month after the date of this report unless other arrangements are made. When making future inquiries regarding this report, please reference the $A C L$ sample number(s) above. For further information about the results reported here, please call L. Chromizky at 2- 4367

Reference(s): Data recorded in Logbook No. 1418, pg. 30; and file folder in cabinet located in Room E-127A.

\begin{tabular}{llll}
\hline Copies To: & D. Analyst(s): L. Chromizky & Draczyk & \\
& M. Robinet & L. Chromizky & \\
D. Geraghty & ACL Result File & \\
lads & D. Green & ACL 200 File & \\
$12 / 16 / 96$ & A. Boparai & &
\end{tabular}


Data File: /users/chem/msdl.i/1dec026.b/c.d Report Date: 10-Dec-1996 15:03

\section{TARGET COMPOUNDS}

Client Name: C. Sholeen

Client Sample ID: 58

Sample Location: Bldg 579

Lab Sample ID: 96-0483-0I

Sample Matrix: Resin

Analysis Type: VOA

Data Type: MS DATA

Misc Info: 58
Client SDG: 1dec026.b

Sample Date:

Sample Point: resin column

Date Received: 09/20/96

Quant Type: ISTD

Leve1: LOW

CAS NO.

COMPOUND

CONCENTRATION UNITS:

(ug/I or $u g / K G) ~ u g / k g$

\begin{tabular}{|c|}
\hline 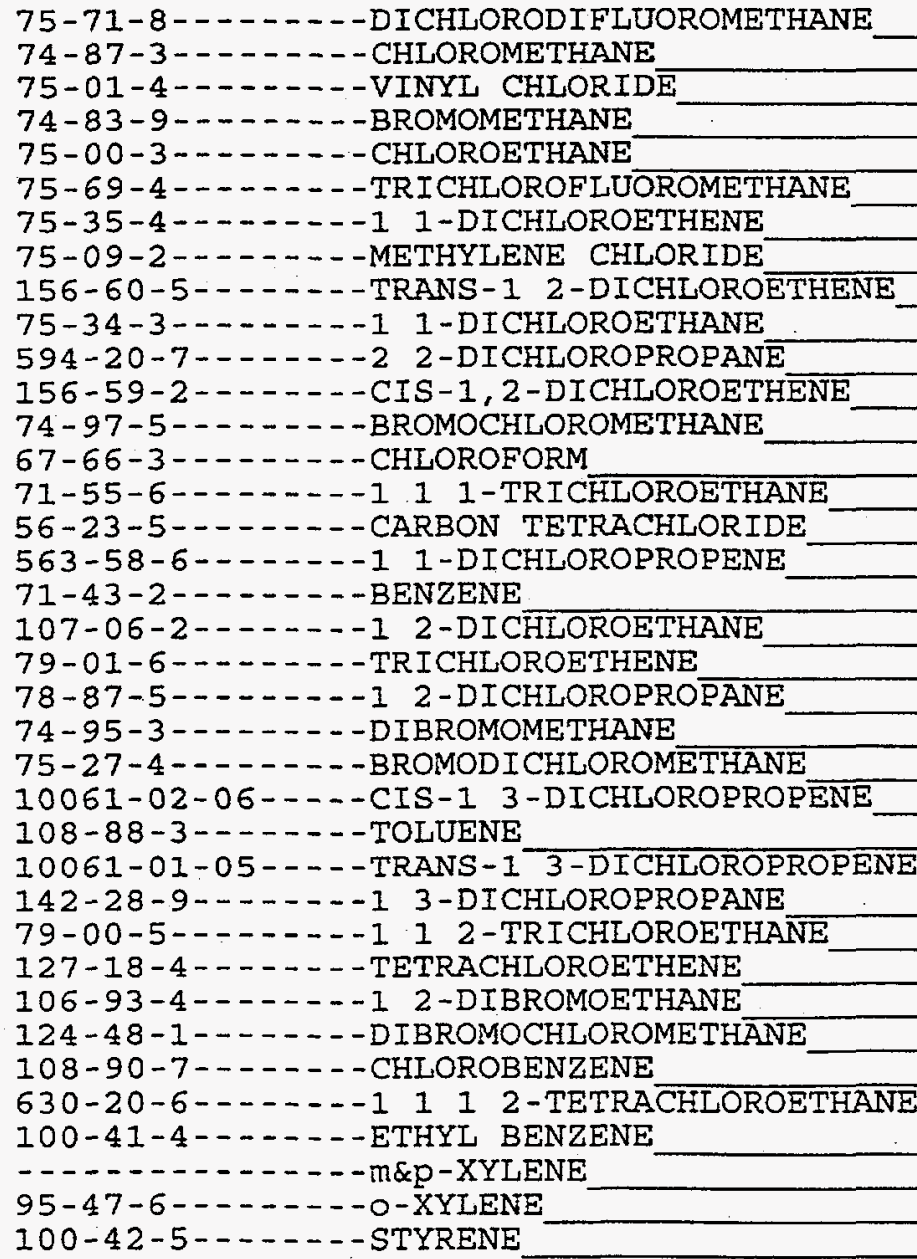 \\
\hline
\end{tabular}

10.00

10.00

10.00

10.00

10.00

10.00

10.00

10.00

10.00

10.00

10.00

10.00

10.00

10.00

10.00

10.00

10.00

10.00

10.00

10.00

10.00

10.00

10.00

10.00

10.00

10.00

10.00

10.00

1.39

10.00

10.00

10.00

10.00

10.00

10.00

10.00

10.00

\begin{tabular}{l}
$U$ \\
$U$ \\
$U$ \\
$U$ \\
$U$ \\
$U$ \\
$U$ \\
$U$ \\
$U$ \\
$U$ \\
$U$ \\
$U$ \\
$U$ \\
$U$ \\
$U$ \\
$U$ \\
$U$ \\
$U$ \\
$U$ \\
$U$ \\
$U$ \\
$U$ \\
$U$ \\
$U$ \\
$U$ \\
$U$ \\
$U$ \\
$U$ \\
$J$ \\
$U$ \\
$U$ \\
$U$ \\
$U$ \\
$U$ \\
$U$ \\
$U$ \\
$U$ \\
\hline
\end{tabular}


Data File: /users/chem/msdl.i/ldec026.b/c.d Report Date: 10-Dec-1996 15:03

\section{TARGET COMPOUNDS}

Client Name: C. Sholeen Client Sample ID: 58 Sample Location: Bldg 579 Lab Sample ID: 96-0483-01 Sample Matrix: Resin Analysis Type: VOA Data Type: MS DATA Misc Info: 58
Client SDG: 1dec026.b Sample Date:

Sample Point: resin column Date Received: 09/20/96

Quant Type: ISTD

Level: LOW

CAS NO.

COMPOUND

CONCENTRATION UNITS :

$(\mathrm{ug} / \mathrm{L}$ or $\mathrm{ug} / \mathrm{KG}) \mathrm{ug} / \mathrm{Kg}$

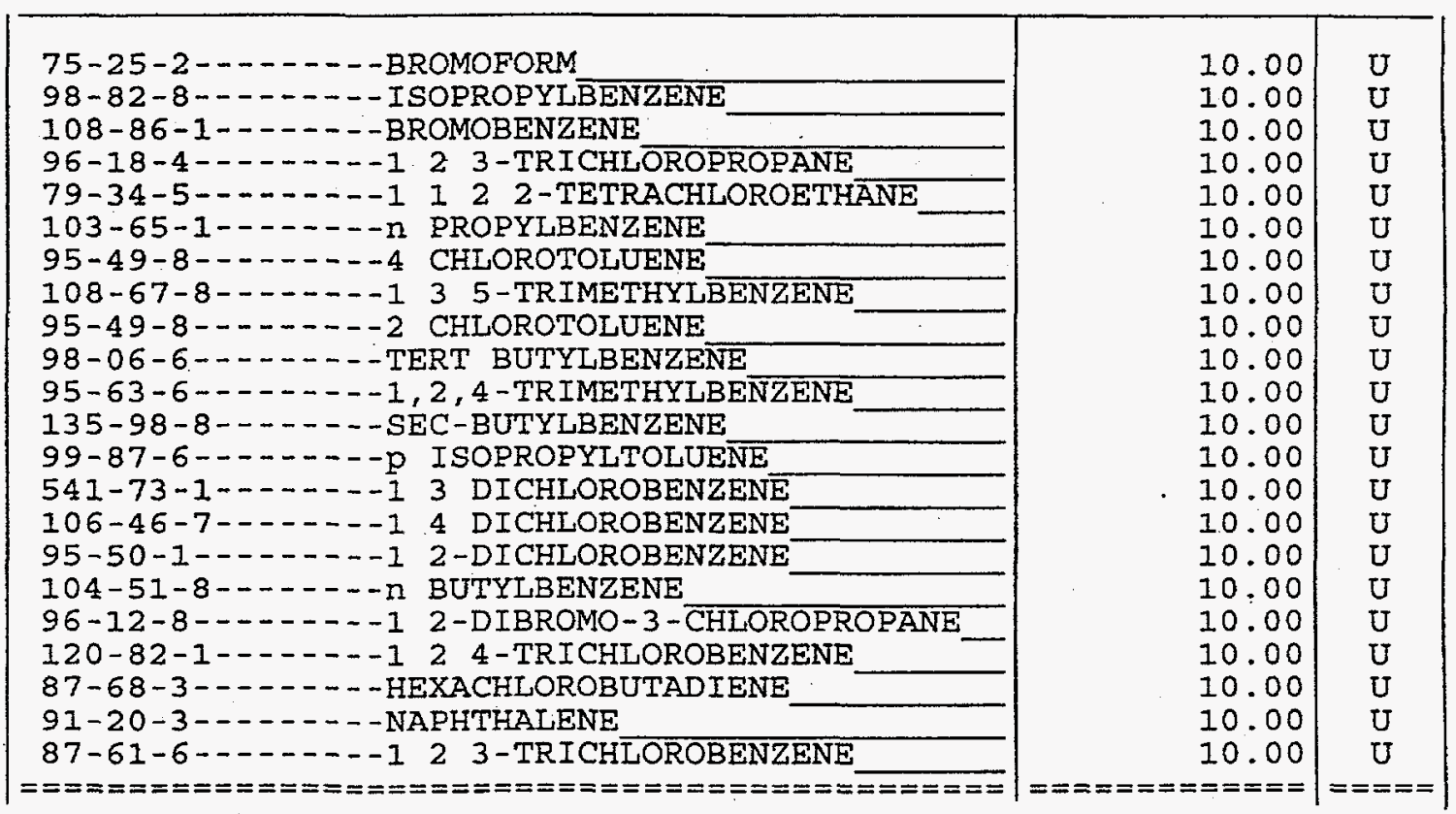


Data File: /users/chem/msdl.i/1dec026.b/c.d Report Date: 10-Dec-1996 15:03

Page 3

\section{RECOVERY REPORT}

Client Name: C. Sholeen

Sample Matrix: Resin

Client SDG: 1dec026.b

Client ID: 58

Data Type: MS DATA

Spikelist File:

Fraction: VOA

Level: LOW

Method File: /

SampleType: SAMPLE

: /users/chem/msdl.i/1dec026.b/rcrav2.m

Misc Info: 58

\begin{tabular}{|c|c|c|c|c|}
\hline SURROGATE COMPOUND & $\begin{array}{l}\text { AMOUNT } \\
\text { ADDED } \\
\text { ug/KG }\end{array}$ & $\begin{array}{l}\text { AMOUNT } \\
\text { RECOVERED } \\
\text { UG/KG }\end{array}$ & $\begin{array}{c}\frac{\circ}{\circ} \\
\text { RECOVERED }\end{array}$ & LIMITS \\
\hline $\begin{array}{lll} & 16 & \text { DIBROMOFLUOROMETHA } \\
\$ & 28 & \text { TOLUENE d-8 } \\
\$ & 45 & 4 \text { BROMOFLUOROBENZE }\end{array}$ & $\begin{array}{l}50.00 \\
50.00 \\
50.00\end{array}$ & $\begin{array}{l}54.35 \\
54.62 \\
55.95\end{array}$ & $\begin{array}{l}108.70^{\circ} \\
109.25 \\
111.91\end{array}$ & $\begin{array}{l}86-118 \\
88-110 \\
86-115\end{array}$ \\
\hline
\end{tabular}

* - Values outside of QC limits

Spike Recovery: 0 out of 3 outside limits

0 out of 3 not found 
ANALYTICAL CHEMISTRY LABORATORY

Argonne National Laboratory

Argonne, IL 60439

\section{REPORT OF ANALYTICAL RESULTS}

$\begin{array}{llll}\text { Sample Material: } & \text { Water } & \text { Date Received: } & 9 / 24 / 96 \\ \text { Submitted by: } & \text { Charlotte Sholeen, ESH-HP, 202 } & \text { Date Reported: } & \text { 10/9/96 }\end{array}$

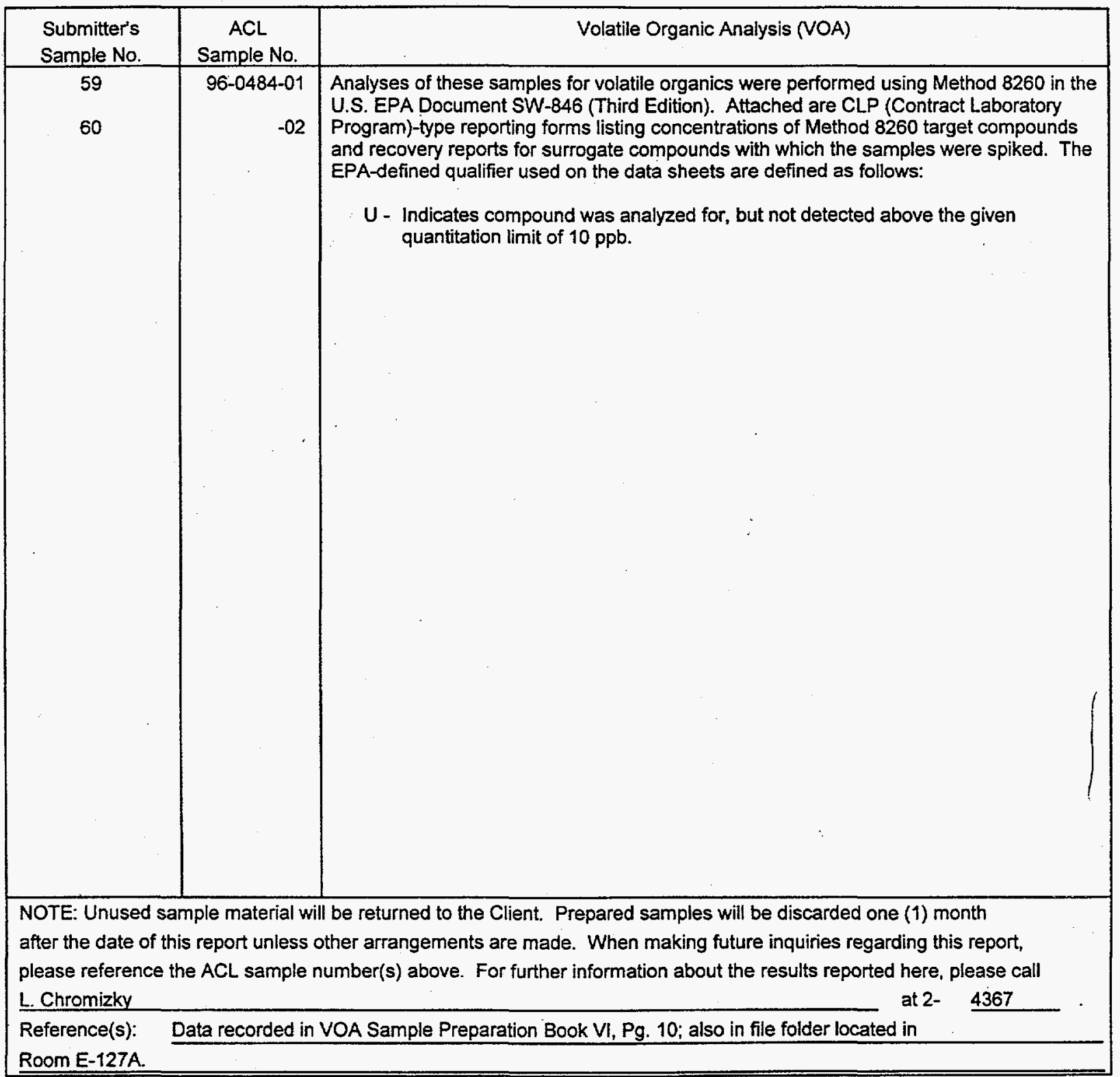

\begin{tabular}{|c|c|c|c|}
\hline Copies To: & $\begin{array}{l}\text { C. Sholeen } \\
\text { D. Geraghty } \\
\text { M. Robinet } \\
\text { D. Green }\end{array}$ & $\begin{array}{l}\text { A. Boparai } \\
\text { D. Graczyk } \\
\text { L. Chromizky } \\
\text { ACL } 200 \text { File }\end{array}$ & Analyst(s): \\
\hline
\end{tabular}

$10 / 9 / 96$

CMT \&4 (9-8) 
Data File: /users/chem/msd1.i/10ct086.b/a.d Report Date: 08-Oct-1996 17:09

TARGET COMPOUNDS

Client Name: C. Sholeen

Client Sample ID: 59

Sample Location: Blig 579

Lab Sample ID: 96-0484-0I

Sample Matrix: WATER

Analysis Type: VOA

Data Type: MS DATA

Misc Info: \#59-Mixed bed exchange column in 579

\author{
Client SDG: 59 \\ Sample Date: \\ Sample Point: \\ Date Received: 09/24/96 \\ Quant Type: ISTD \\ Level: LOW
}

$\begin{array}{ll}\text { CAS NO. COMPOUND } & \text { CONCENTRATION UNITS : } \\ \text { (ug/L or ug/KG) ug/I }\end{array}$
Q
10.00

10.00

10.00

10.00

10.00

10.00

10.00

10.00

10.00

10.00

10.00

10.00

10.00

10.00

10.00

10.00

10.00

10.00

10.00

10.00

10.00

10.00

10.00

10.00

10.00

10.00

10.00

10.00

10.00

10.00

10.00

10.00

10.00

10.00

10.00

10.00

10.00

\begin{tabular}{l}
$U$ \\
$U$ \\
$U$ \\
$U$ \\
$U$ \\
$U$ \\
$U$ \\
$U$ \\
$U$ \\
$U$ \\
$U$ \\
$U$ \\
$U$ \\
$U$ \\
$U$ \\
$U$ \\
$U$ \\
$U$ \\
$U$ \\
$U$ \\
$U$ \\
$U$ \\
$U$ \\
$U$ \\
$U$ \\
$U$ \\
$U$ \\
$U$ \\
$U$ \\
$U$ \\
$U$ \\
$U$ \\
$U$ \\
$U$ \\
$U$ \\
$U$ \\
$U$ \\
\hline
\end{tabular}




\section{TARGET COMPOUNDS}

Client Name: C. Sholeen

Client Sample ID: 59

Sample Location: Bldg 579

Lab Sample ID: 96-0484-01

Sample Matrix: WATER

Analysis Type: VOA

Data Type: MS DATA

Misc Info: \#59-Mixed bed exchange column in 579
Client SDG: 59

Sample Date:

Sample Point:

Date Received: 09/24/96

Quant Type: ISTD

Level: LOW

CAS NO.

COMPOUND

CONCENTRATION UNITS:

(ug/L or ug/KG) ug/L

\begin{tabular}{|c|c|c|}
\hline $\begin{array}{ll}75-25-2 & -1-1 \\
98-82-8 & -1\end{array}$ & $\begin{array}{l}10.00 \\
10.00 \\
10.00 \\
10.00 \\
10.00 \\
10.00 \\
10.00 \\
10.00 \\
10.00 \\
10.00 \\
10.00 \\
10.00 \\
10.00 \\
10.00 \\
10.00 \\
10.00 \\
10.00 \\
10.00 \\
10.00 \\
10.00 \\
10.00 \\
10.00 \\
===== \\
54.35 \\
51.01 \\
43.79\end{array}$ & $\begin{array}{l}\mathrm{U} \\
\mathrm{U} \\
\mathrm{U} \\
\mathrm{U} \\
\mathrm{U} \\
\mathrm{U} \\
\mathrm{U} \\
\mathrm{U} \\
\mathrm{U} \\
\mathrm{U} \\
\mathrm{U} \\
\mathrm{U} \\
\mathrm{U} \\
\mathrm{U} \\
\mathrm{U} \\
\mathrm{U} \\
\mathrm{U} \\
\mathrm{U} \\
\mathrm{U} \\
\mathrm{U} \\
\mathrm{U} \\
\mathrm{U} \\
====\end{array}$ \\
\hline
\end{tabular}


Data File: /users/chem/msd1.i/1oct086.b/a.d Report Date: 08-Oct-1996 17:09

\section{RECOVERY REPORT}

Client Name: C. Sholeen

Sample Matrix: LIQUID

Client ID: 59

Data Type: MS DATA

Spikelist File:
Method File:/users/chem/msd1.i/loct 086 .b/rcrav2.m

Client SDG: 59

Fraction: VOA

Level: LOW

SampleType: SAMPLE

Misc Info: \#59-Mixed bed exchange column in 579

\begin{tabular}{|c|c|c|c|c|}
\hline SURROGATE COMPOUND & $\begin{array}{l}\text { AMOUNT } \\
\text { ADDED }\end{array}$ & $\begin{array}{c}\text { AMOUNT } \\
\text { RECOVERED }\end{array}$ & RECOVERED & LIMITS \\
\hline $\begin{array}{lll}\text { \$ } & 16 & \text { DIBROMOFLUOROMETHA } \\
\$ & 28 & \text { TOLUENE } d-8 \\
\$ & 45 & 4 \text { BROMOFLUOROBENZE }\end{array}$ & $\begin{array}{l}50.00 \\
50.00 \\
50.00\end{array}$ & $\begin{array}{l}54.35 \\
51.01 \\
43.79\end{array}$ & $\begin{array}{r}108.70 \\
102.03 \\
87.58\end{array}$ & $\begin{array}{l}\overline{86-118} \\
88-110 \\
86-115\end{array}$ \\
\hline
\end{tabular}

* - Values outside of QC Iimits

Spike Recovery: 0 out of 3 outside limits

0 out of 3 not found 


\section{TARGET COMPOUNDS}

Client Name: C. Sholeen

Client Sample ID: 60

Sample Location: Bldg 579

Lab Sample ID: 96-0484-02

Sample Matrix: WATER

Analysis Type: VOA

Data Type: MS DATA

Misc Info: \#60-Drain tank in bldg 579
Client SDG: 59

Sample Date:

Sample Point:

Date Received: 09/24/96

Quant Type: ISTD

Level: LOW
CAS NO.

COMPOUND

CONCENTRATION UNITS: (ug/L or $\mathrm{ug} / \mathrm{KG}$ ) ug/L

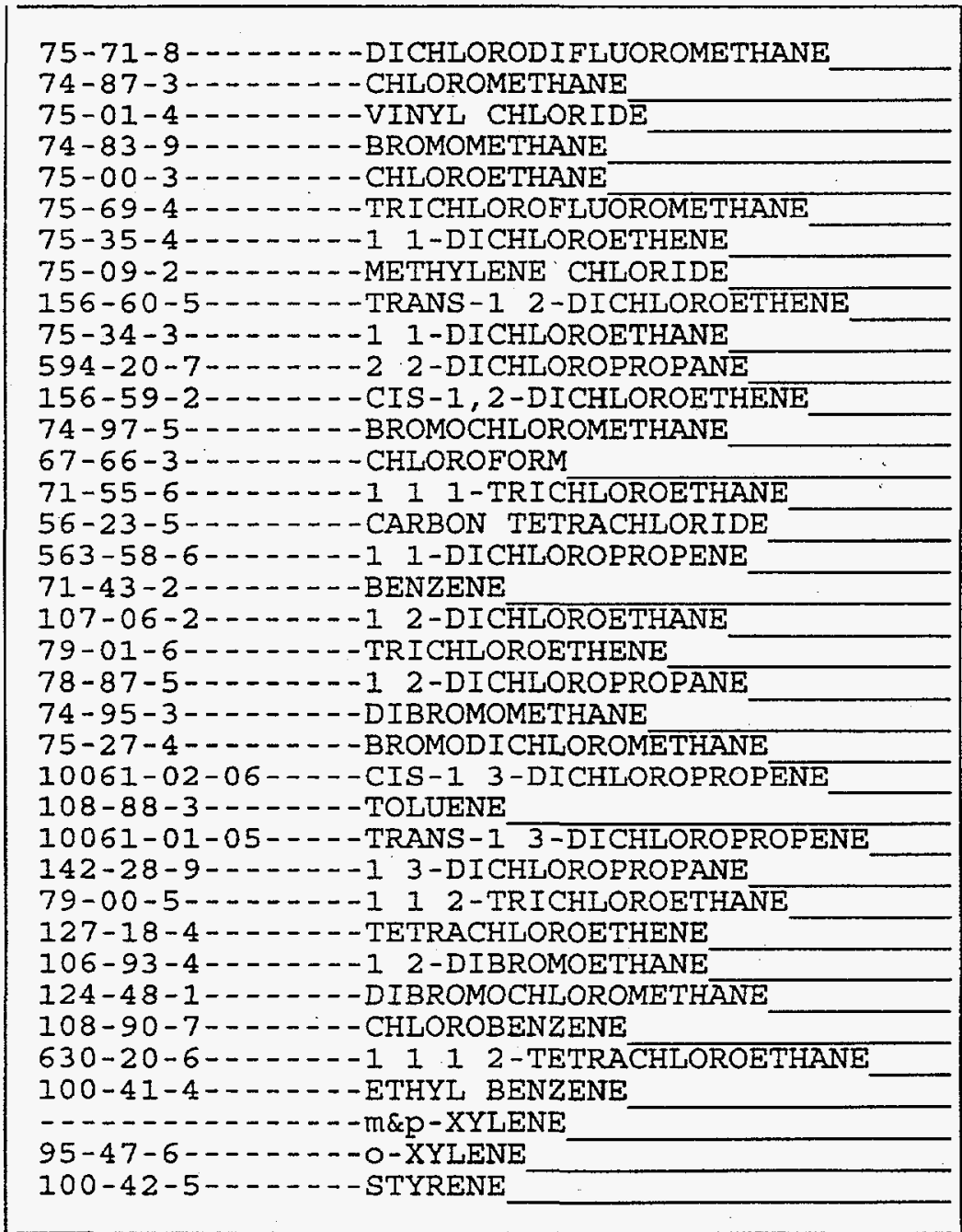

10.00

10.00

10.00

10.00

10.00

10.00

10.00

10.00

10.00

10.00

10.00

$10: 00$

10.00

10.00

10.00

10.00

10.00

10.00

10.00

10.00

10.00

$10: 00$

10.00

10.00

10.00

10.00

10.00

10.00

10.00

10.00

10.00

10.00

10.00

10.00

10.00

10.00

10.00

\begin{tabular}{l}
$U$ \\
$U$ \\
$U$ \\
$U$ \\
$U$ \\
$U$ \\
$U$ \\
$U$ \\
$U$ \\
$U$ \\
$U$ \\
$U$ \\
$U$ \\
$U$ \\
$U$ \\
$U$ \\
$U$ \\
$U$ \\
$U$ \\
$U$ \\
$U$ \\
$U$ \\
$U$ \\
$U$ \\
$U$ \\
$U$ \\
$U$ \\
$U$ \\
$U$ \\
$U$ \\
$U$ \\
$U$ \\
$U$ \\
$U$ \\
$U$ \\
$U$ \\
$U$ \\
\hline
\end{tabular}


Data File: /users/chem/msdl.i/10ct086.b/b.d Report Date: 08-0ct-1996 17:09

Page 3

\section{TARGET COMPOUNDS}

Client Name: C. Sholeen

Client Sample ID: 60

Sample Location: Bldg 579

Lab Sample ID: 96-0484-02

Sample Matrix: WATER

Analysis Type: VOA

Data Type: MS DATA

Misc Info: \#60-Drain tank in bldg 579
Client SDG: 59

Sample Date:

Sample Point:

Date Received: 09/24/96

Quant Type: ISTD

Level: LOW

CAS NO.

COMPOUND

CONCENTRATION UNITS:

(ug/L or ug/KG) ug/L

Q

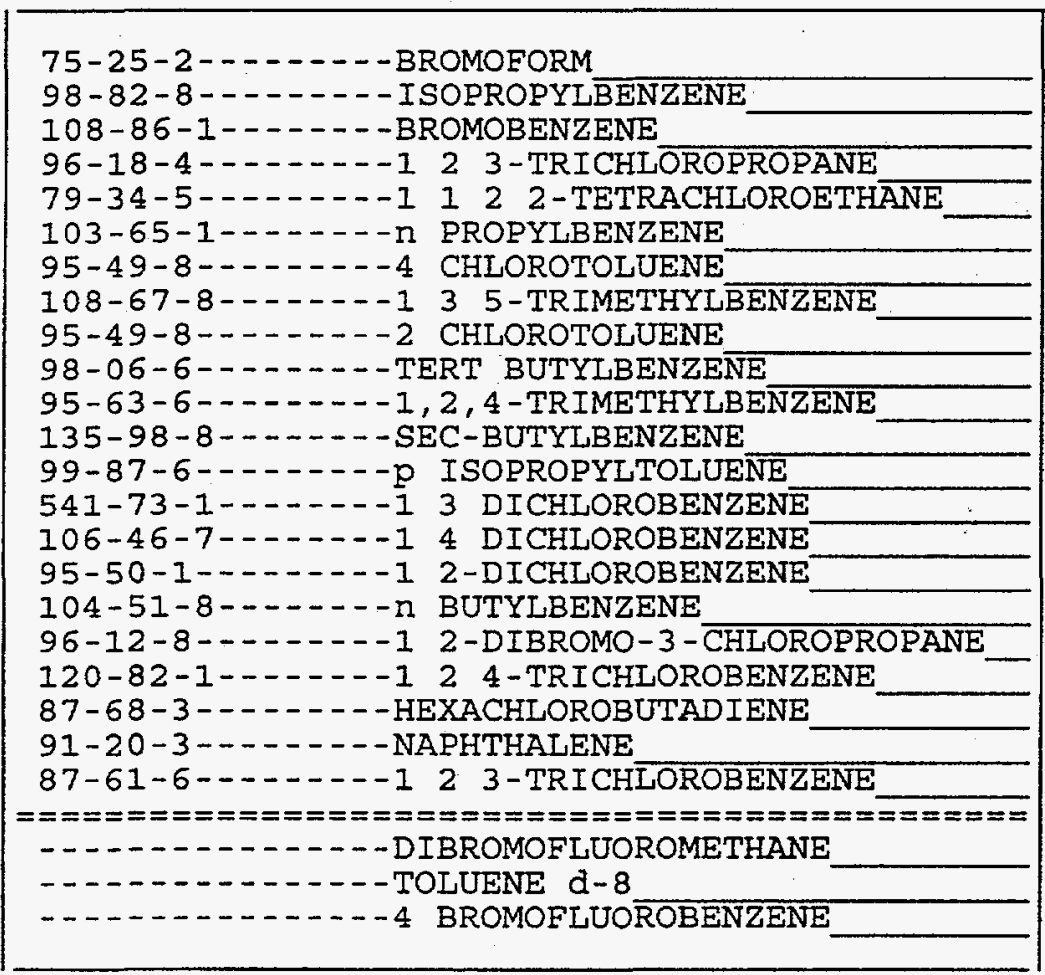

\begin{tabular}{c|c}
10.00 & $\mathrm{U}$ \\
10.00 & $\mathrm{U}$ \\
10.00 & $\mathrm{U}$ \\
10.00 & $\mathrm{U}$ \\
10.00 & $\mathrm{U}$ \\
10.00 & $\mathrm{U}$ \\
10.00 & $\mathrm{U}$ \\
10.00 & $\mathrm{U}$ \\
10.00 & $\mathrm{U}$ \\
10.00 & $\mathrm{U}$ \\
10.00 & $\mathrm{U}$ \\
10.00 & $\mathrm{U}$ \\
10.00 & $\mathrm{U}$ \\
10.00 & $\mathrm{U}$ \\
10.00 & $\mathrm{U}$ \\
10.00 & $\mathrm{U}$ \\
10.00 & $\mathrm{U}$ \\
10.00 & $\mathrm{U}$ \\
10.00 & $\mathrm{U}$ \\
10.00 & $\mathrm{U}$ \\
10.00 & $\mathrm{U}$ \\
10.00 & $\mathrm{U}$ \\
$5====$ & $=====$ \\
52.81 & \\
49.72 & \\
43.17 & \\
\hline
\end{tabular}


Data File: /users/chem/msdl.i/10ct086.b/b.d

Report Date: 08-Oct-1996 17:09

RECOVERY REPORT

Client Name: C. Sholeen

Sample Matrix: IIQUID

Client ID: 60

Data Type: MS DATA

Spikelist File:

Method File:/users/chem/msdl.i/10ct086.b/rcrav2.m

Client SDG: 59

Fraction: VOA

Level: LOW

SampleType: SAMPLE

Quant Type: ISTD

Misc Info: \#60-Drain tank in bldg 579

\begin{tabular}{|c|c|c|c|c|}
\hline SURROGATE COMPOUND & $\begin{array}{l}\text { AMOUNT } \\
\text { ADDED } \\
\text { ug/I }\end{array}$ & $\begin{array}{l}\text { AMOUNT } \\
\text { RECOVERED } \\
\text { UG/L }\end{array}$ & RECOVERED & IIMITS \\
\hline $\begin{array}{lll}\$ & 16 & \text { DIBROMOFLUOROMETHA } \\
\$ & 28 & \text { TOLUENE d-8 } \\
\$ & 45 & 4 \text { BROMOFLUOROBENZE }\end{array}$ & $\begin{array}{l}50.00 \\
50.00 \\
50.00\end{array}$ & $\begin{array}{l}52.81 \\
49.72 \\
43.17\end{array}$ & $\begin{array}{r}105.61 \\
99.45 \\
86.35\end{array}$ & $\begin{array}{l}86-118 \\
88-110 \\
86-115\end{array}$ \\
\hline
\end{tabular}

* - Values outside of QC Iimits

Spike Recovery: 0 out of 3 outside Iimits

0 out of 3 not found 
ANALYTICAL CHEMISTRY LABORATORY

Argonne National Laboratory

Argonne, IL. 60439

REPORT OF ANALYTICAL RESULTS

\begin{tabular}{lllr} 
Sample Material: & Resin & Date Received: & $9 / 20 / 96$ \\
Submitted by: & Charlotte Sholeen, ESH-HP, 202 & Date Reported: & 12/6/96 \\
\hline
\end{tabular}

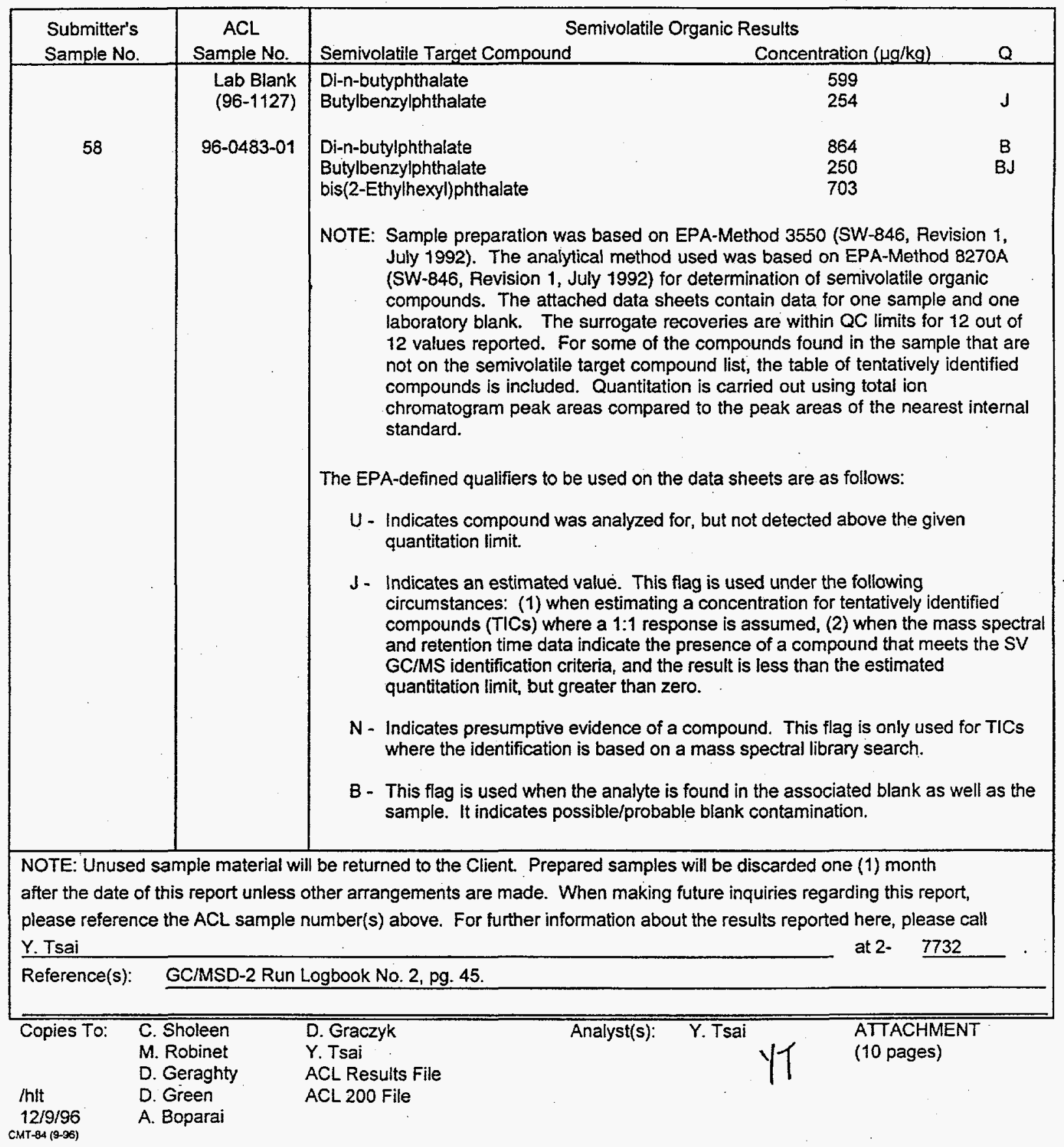


Data File: /data/msd2.i/2dec046.b/0101001.d Report Date: 05-Dec-1996 13:30

ANL

\section{TARGET COMPOUNDS}

Client Name:

Lab Smp Id: 96-1127, Lab Blank

Sample Location:

Sample Date:

Sample Matrix: SOIL

Analysis TYpe: SV

Data Type: MS DATA

Misc Info:
Client SDG: 2 dec $046 . \mathrm{b}$

Client Smp ID: Lab Blank

Sample Point:

Date Received:

Quant Type: ISTD

Level: LOW

Operator: Y. Tsai

CAS NO.

COMPOUND

CONCENTRATION UNITS:

$(\mathrm{ug} / \mathrm{I}$ or $\mathrm{ug} / \mathrm{KG}) \mathrm{ug} / \mathrm{Kg}$

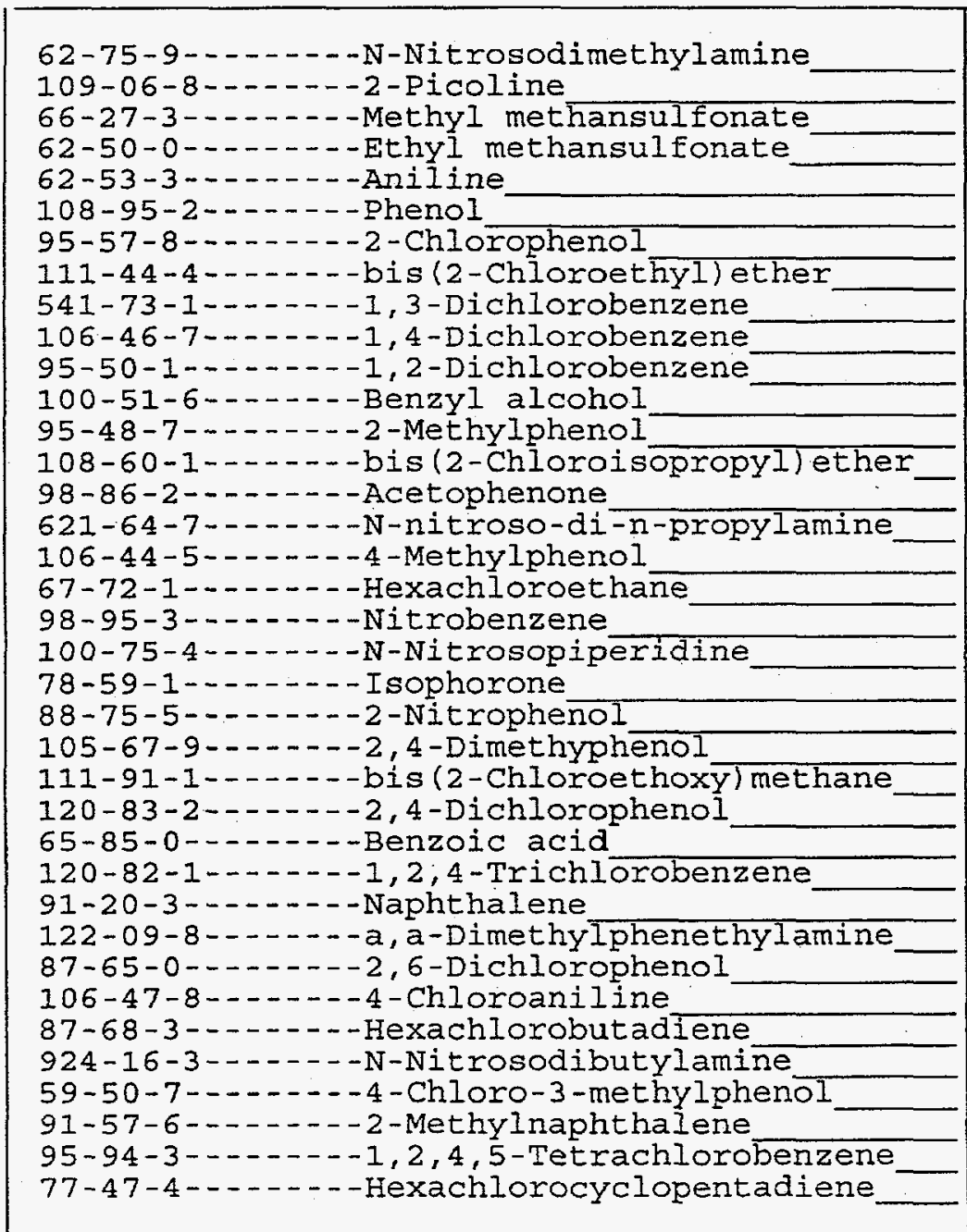

\begin{tabular}{|l|l|}
330 & $\mathrm{U}$ \\
330 & $\mathrm{U}$ \\
660 & $\mathrm{U}$ \\
330 & $\mathrm{U}$ \\
330 & $\mathrm{U}$ \\
330 & $\mathrm{U}$ \\
330 & $\mathrm{U}$ \\
330 & $\mathrm{U}$ \\
330 & $\mathrm{U}$ \\
330 & $\mathrm{U}$ \\
330 & $\mathrm{U}$ \\
330 & $\mathrm{U}$ \\
330 & $\mathrm{U}$ \\
330 & $\mathrm{U}$ \\
330 & $\mathrm{U}$ \\
330 & $\mathrm{U}$ \\
330 & $\mathrm{U}$ \\
330 & $\mathrm{U}$ \\
330 & $\mathrm{U}$ \\
330 & $\mathrm{U}$ \\
330 & $\mathrm{U}$ \\
330 & $\mathrm{U}$ \\
330 & $\mathrm{U}$ \\
330 & $\mathrm{U}$ \\
330 & $\mathrm{U}$ \\
330 & $\mathrm{U}$ \\
330 & $\mathrm{U}$ \\
330 & $\mathrm{U}$ \\
660 & $\mathrm{U}$ \\
330 & $\mathrm{U}$ \\
330 & $\mathrm{U}$ \\
330 & $\mathrm{U}$ \\
330 & $\mathrm{U}$ \\
330 & $\mathrm{U}$ \\
330 & $\mathrm{U}$ \\
330 & $\mathrm{U}$ \\
660 & $\mathrm{U}$ \\
& \\
\hline & \\
\hline
\end{tabular}


Data File: /data/msd2.i/2dec046.b/0101001.d Report Date: 05-Dec-1996 13:30

ANL TARGET COMPOUNDS

Client Name:

Lab Smp Id: 96-1127, Lab Blank

Sample Location:

Sample Date:

Sample Matrix: SOII

Analysis Type: SV

Data Type: MS DATA

Misc Info:
Client SDG: 2dec046.b

Client Smp ID: Lab Blank

Sample Point:

Date Received:

Quant Type: ISTD

Level: LOW

Operator: $Y$. Tsai

CAS NO. COMPOUND

CONCENTRATION UNITS :

(ug/L or $\mathrm{ug} / \mathrm{KG}$ ) ug/ $\mathrm{Kg}$

88-06-2-....--2,4,6-Trichlorophenol

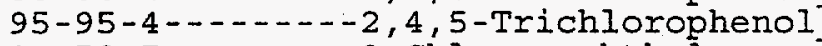

91-58-7---.-2-Chloronaphthalene

90-13-1-...-1-Chloronaphthalene

88-74-4-------2-Nitroaniline

131-11-3-...--Dimethyl phthalate

208-96-8--.---Acenaphthylene

606-20-2--.---2,6-Dinitrotoluene

- - - - Nitroaniline

83-32-9-1-n--Acenaphthene

51-28-5------2, 4-Dinitrophenol

608-93-5------Pentachlorobenzene

132-64-9-...-Dibenzofuran

100-02-7--.---4-NitrophenoI

121-14-2-..--2,4-Dinitrotoluene

91-59-8---.--2-Napthylamine

58-90-2 -....-2,3,4,6-Tetrachlorophenol

$134-32-7-\cdots--1-$ Naphthylamine

86-73-7--.---- Fluorene

84-66-2--.---Diethylphthalate

7005-72-3---1-4-Chlorophenyl phenyl ether

100-01-6-...-.-4-Nitroaniline

534-52-1------4,6-Dinitro-2-methylphenol

-...-NitrosoDPA \& Diphenylamine

122-66-7--.--1,2-Diphenylhydrazine

101-55-3-1-1-4-Bromophenyl phenyl ether

118-74-1-....--Hexachlorobenzene

$62-44-2-\cdots----$ Phenacetin

87-86-5-1.-.--Pentachlorophenol

82-68-8-...---Pentachloronitrobenzene

92-67-1-1-.--4-Aminobiphenyl

23950-58-5-----Pronamide

85-01-8--.-.--Phenanthrene

120-12-7-...-Anthracene

84-74-2------Di-n-butylphthalate

206-44-0-...-.-Fluoranthene

$92-87-5-\ldots-\cdots-$ - Benzidine 
Data File:/data/msd2.i/2dec046.b/0101001.d Report Date: 05-Dec-1996 13:30

ANL

\section{TARGET COMPOUNDS}

Client Name:

Lab Smp Id: 96-1127, Lab Blank Sample Location:

Sample Date:

Sample Matrix: SOIL

Analysis Type: SV

Data Type: MS DATA

Misc Info:
Client SDG: 2dec046.b Client Smp ID: Irab Blank Sample point:

Date Received:

Quant Type: ISTD

Level: LOW

Operator: Y. Tsai

CAS NO.

COMPOUND

CONCENTRATION UNITS:

( $\mathrm{ug} / \mathrm{L}$ or $\mathrm{ug} / \mathrm{KG}$ ) $\mathrm{ug} / \mathrm{Kg}$

\begin{tabular}{|c|c|c|}
\hline 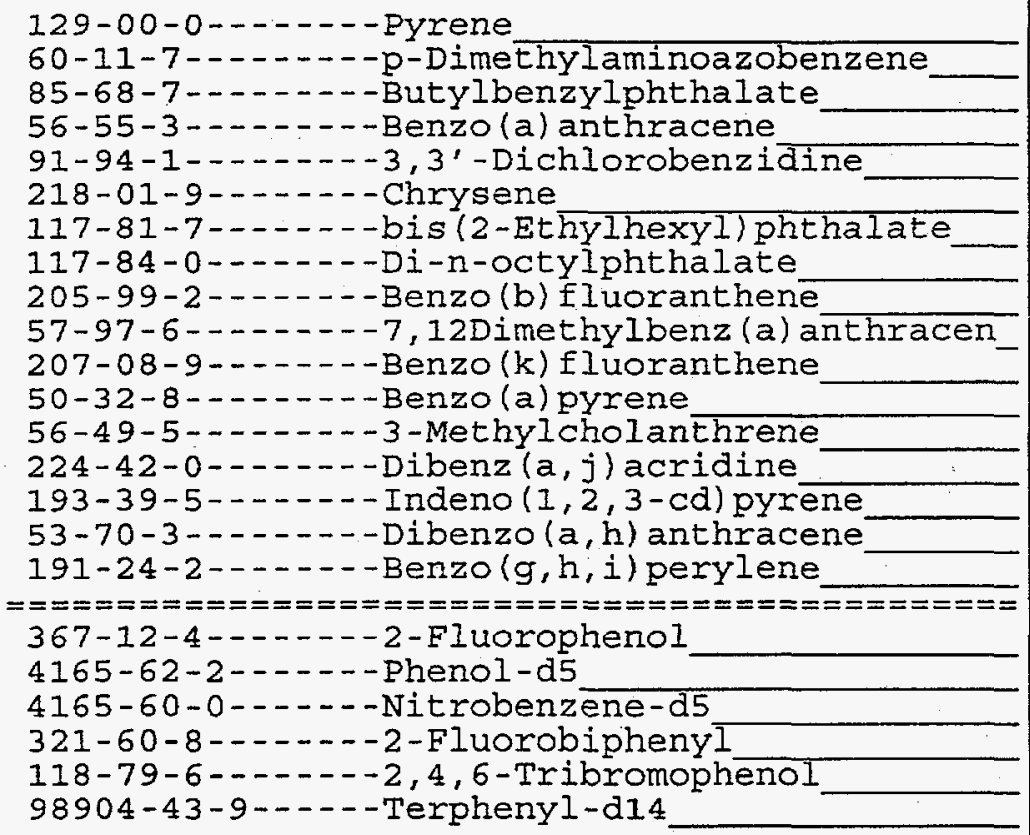 & $\begin{array}{r}330 \\
330 \\
254 \\
330 \\
330 \\
330 \\
330 \\
330 \\
330 \\
330 \\
330 \\
330 \\
330 \\
330 \\
330 \\
330 \\
330 \\
========1 \\
5320 \\
6130 \\
2770 \\
3140 \\
6680 \\
2390\end{array}$ & $\begin{array}{l}U \\
U \\
J \\
U \\
U \\
U \\
U \\
U \\
U \\
U \\
U \\
U \\
U \\
U \\
U \\
U \\
U \\
=====\end{array}$ \\
\hline
\end{tabular}


Data File: /data/msd2.i/2dec046.b/0101001.d

Report Date: 05-Dec-1996 13:30

ANL

\section{RECOVERY REPORT}

Client Name:

Sample Matrix: SOLID

Lab Smp Id: 96-1127, Lab Blank

Level: LOW

Data Type: MS DATA

SpikeList File: HPMSS.spk

Method File: /data/msd2 $i / 2$ dec046.b/Yt82
Client SDG: 2dec046.b

Fraction: SV

Client Smp ID: Lab Blank

Operator: Y. Tsai

SampleType: BLANK

Quant Type: ISTD

Misc Info:

\begin{tabular}{|c|c|c|c|c|}
\hline SURROGATE COMPOUND & $\begin{array}{l}\text { AMOUNT } \\
\text { ADDED }\end{array}$ & $\begin{array}{c}\text { AMOUNT } \\
\text { RECOVERED }\end{array}$ & $\begin{array}{c}\frac{\circ}{\circ} \\
\text { RECOVERED }\end{array}$ & LIMITS \\
\hline $\begin{array}{lrl}\text { \$ } & 4 & 2-\text { Fluorophenol } \\
\$ & 7 & \text { Phenol-d5 } \\
\text { \$ } & 22 & \text { Nitrobenzene-d5 } \\
\$ & 45 & 2-\text { Fluorobiphenyl } \\
\text { \$ } & 70 & 2,4,6-T r i b r o m o p h e n \\
\$ & 85 & \text { Terphenyl-d14 }\end{array}$ & $\begin{array}{l}200.00 \\
200.00 \\
100.00 \\
100.00 \\
200.00 \\
100.00\end{array}$ & $\begin{array}{r}159.71 \\
184.01 \\
83.16 \\
94.17 \\
200.52 \\
71.84\end{array}$ & $\begin{array}{r}79.85 \\
92.01 \\
83.16 \\
94.17 \\
100.26 \\
71.84\end{array}$ & $\begin{array}{l}25-121 \\
24-113 \\
23-120 \\
30-115 \\
19-122 \\
18-137\end{array}$ \\
\hline
\end{tabular}

* - Values outside of QC Iimits

Spike Recovery: 0 out of 6 outside limits

0 out of 6 not found 
Data File: /data/msd2.i/2dec046.b/1601016.d Report Date: 06-Dec-1996 14:06

\section{TARGET COMPOUNDS}

Client Name: C. Sholeen

Client Sample ID: 58 (Resin)

Sample Location:

Lab Sample ID: 96-0483-01

Sample Matrix: SOIL

Analysis Type: SV

Data Type: MS DATA

Misc Info:

\author{
Client SDG: 2 dec0 $046 . b$ \\ Sample Date: \\ Sample Point: \\ Date Received: \\ Quant Type: ISTD \\ Level: LOW
}

CAS NO.

COMPOUND
CONCENTRATION UNITS:

$(\mathrm{ug} / \mathrm{L}$ or $\mathrm{ug} / \mathrm{KG}) \mathrm{ug} / \mathrm{Kg}$
Q

\begin{tabular}{|c|}
\hline 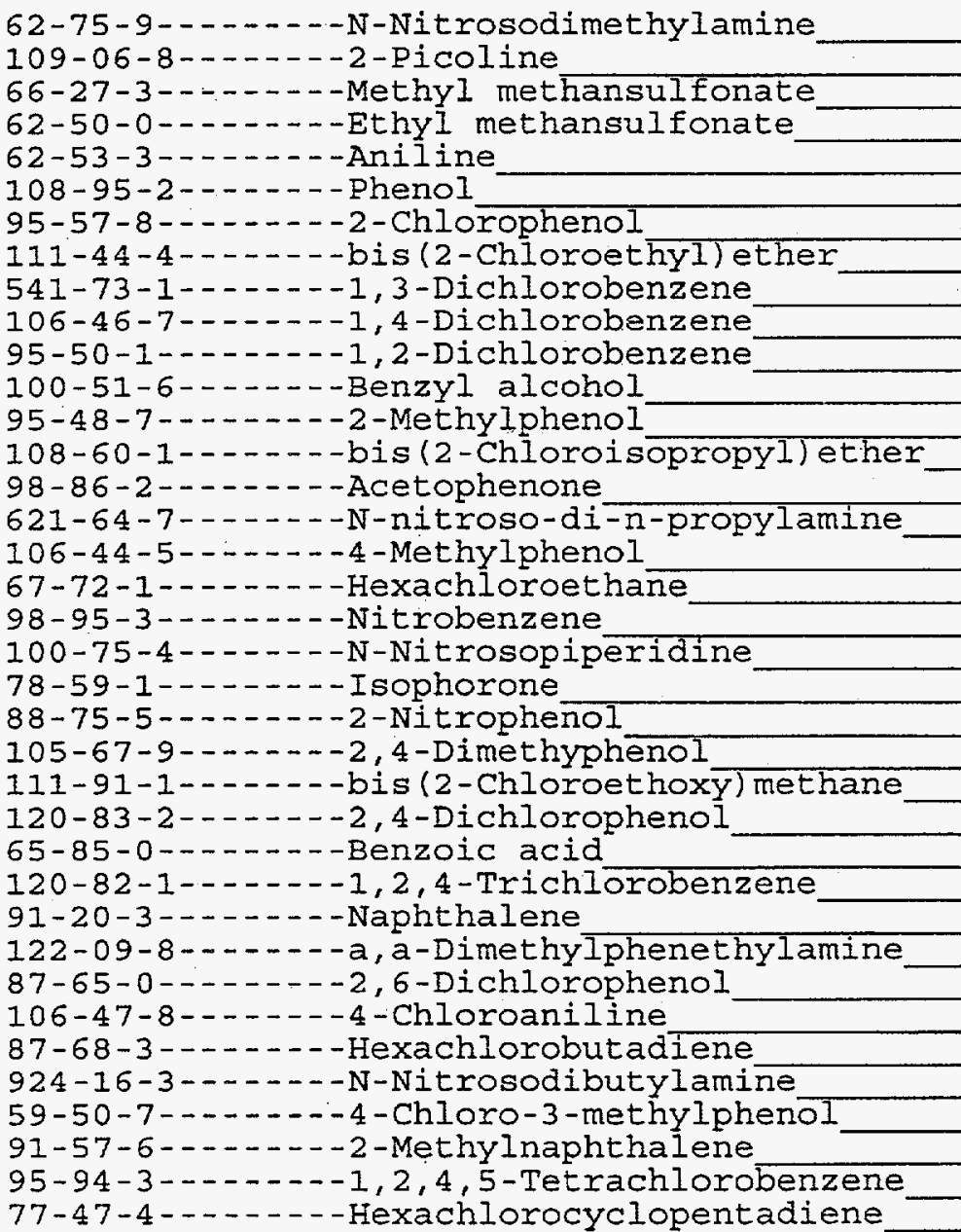 \\
\hline
\end{tabular}


Data File: /data/msd2.i/2dec046.b/1601016.d Report Date: 06-Dec-1996 14:06

\section{TARGET COMPOUNDS}

Client Name: C. Sholeen

Client Sample ID: 58 (Resin)

Sample Location:

Lab Sample ID: 96-0483-01

Sample Matrix: SOIL

Analysis Type: SV

Data TYpe: MS DATA

Misc Info:

\author{
Client SDG: 2dec046.b \\ Sample Date: \\ Sample Point: \\ Date Received: \\ Quant Type: ISTD \\ Level: LOW
}

CAS NO.

COMPOUND

CONCENTRATION UNITS :

(ug/L or $\mathrm{ug} / \mathrm{KG}) \mathrm{ug} / \mathrm{Kg}$

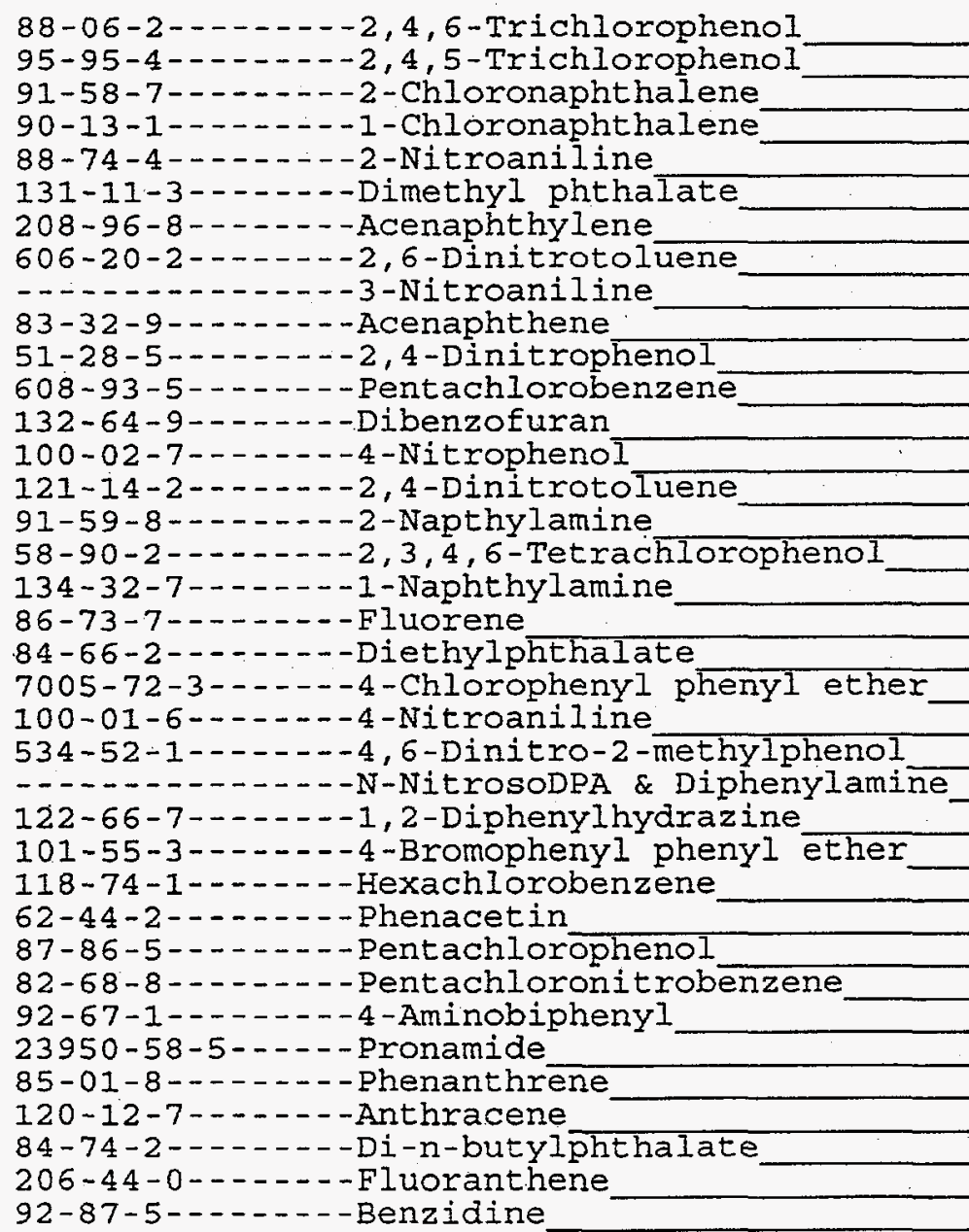

92-87-5-a-zane 
Data File:/data/msd2.i/2dec046.b/1601016.d Report Date: 06-Dec-1996 14:06

\section{TARGET COMPOUNDS}

Client Name: C. Sholeen

Client Sample ID: 58 (Resin)

Sample Location:

Lab Sample ID: 96-0483-01

Sample Matrix: SOIL

Analysis Type: SV

Data Type: MS DATA

Misc Info:
Client SDG: 2dec046.b

Sample Date:

Sample Point:

Date Received:

Quant TYpe: ISTD

Level: LOW

CAS NO.

COMPOUND

CONCENTRATION UNITS:

(ug/L or $\mathrm{ug} / \mathrm{KG}$ ) ug/Kg

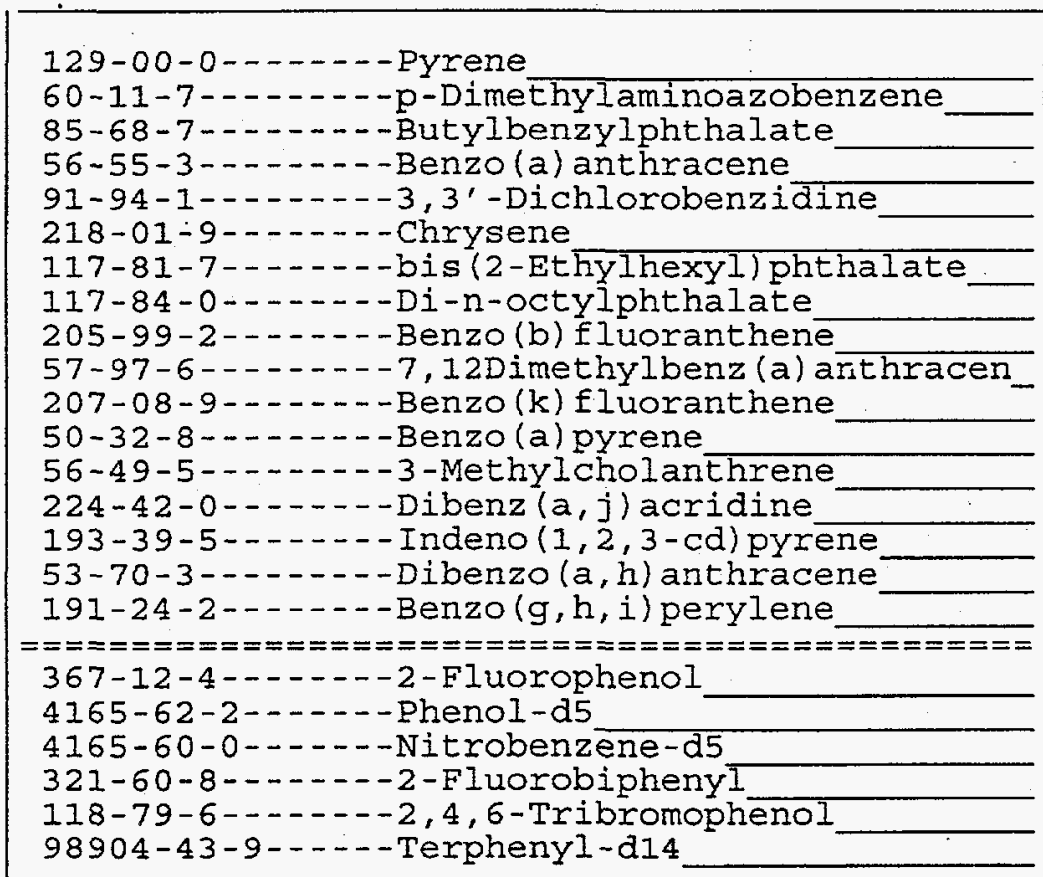

\begin{tabular}{|c|c|}
330 & $\mathrm{U}$ \\
330 & $\mathrm{U}$ \\
250 & $\mathrm{JB}$ \\
330 & $\mathrm{U}$ \\
330 & $\mathrm{U}$ \\
330 & $\mathrm{U}$ \\
703 & \\
330 & $\mathrm{U}$ \\
330 & $\mathrm{U}$ \\
330 & $\mathrm{U}$ \\
330 & $\mathrm{U}$ \\
330 & $\mathrm{U}$ \\
330 & $\mathrm{U}$ \\
330 & $\mathrm{U}$ \\
330 & $\mathrm{U}$ \\
330 & $\mathrm{U}$ \\
330 & $\mathrm{U}$ \\
$===$ & $====$ \\
3670 & \\
4170 & \\
1740 & \\
1890 & \\
4680 & \\
2870 & \\
\hline
\end{tabular}


Data File: /data/msd2.i/2dec046.b/1601016.d Report Date: 06-Dec-1996 14:06

\section{RECOVERY REPORT}

Client Name: C. Sholeen

Sample Matrix: SOLID

Client ID: 58 (Resin)

Data Type: MS DATA

Spikelist File: HPMSS.spk

Method File: /data/msd2.i/2dec046.b/yt8270.m

Client SDG: 2dec046.b

Fraction: SV

Level: LOW

SampleType: SAMPLE

Quant Type: ISTD

Misc Info:

\begin{tabular}{|c|c|c|c|c|}
\hline SURROGATE COMPOUND & $\begin{array}{l}\text { AMOUNNT } \\
\text { ADDED }\end{array}$ & $\begin{array}{c}\text { AMOUNT } \\
\text { RECOVERED }\end{array}$ & RECOVERED & LIMITS \\
\hline $\begin{array}{rrl} & 4 & 2-\text { Fluorophenol } \\
\$ & 7 & \text { Phenol-d5 } \\
\$ & 22 & \text { Nitrobenzene-d5 } \\
\$ & 45 & 2-\text { Fluorobiphenyl } \\
\$ & 70 & 2,4,6-\text { Tribromophen } \\
\$ & 85 & \text { Terphenyl-d14 }\end{array}$ & $\begin{array}{l}200 \\
200 \\
100 \\
100 \\
200 \\
100\end{array}$ & $\begin{array}{r}110 \\
125 \\
52.2 \\
56.6 \\
140 \\
86.1\end{array}$ & $\begin{array}{l}55 \cdot 12 \\
62.59 \\
52.21 \\
56.60 \\
70.19 \\
86.11\end{array}$ & $\begin{array}{l}25-121 \\
24-113 \\
23-120 \\
30-115 \\
19-122 \\
18-137\end{array}$ \\
\hline
\end{tabular}

* - Values outside of QC limits

Spike Recovery: 0 out of 6 outside limits

0 out of 6 not found 
Data File: /data/msd2.i/2dec046.b/1601016.d

Report Date: 12/06/96 14:06

Client Name: C. Sholeen

Lab Smp ID: 96-0483-01

Operator: Y. Tsai

Sample Location:

Sample Matrix: SOIL

Analysis Type: SV
Client SDG: 2dec046.b

Client Smp ID: 58 (Resin)

Sample Date:

Sample Point:

Date Received:

Level: Low
Number TICs found: 22

\begin{tabular}{|c|c|c|c|c|c|}
\hline 1 & I & 1 & 1 & I & I \\
\hline CAS NUMBER & COMPOUND NAME & $\mathbf{R T}$ & 1 & Conc. & $Q$ \\
\hline \multicolumn{6}{|c|}{ 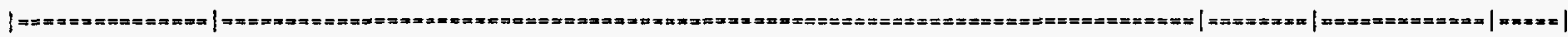 } \\
\hline 1 1. 1193-11-9 & 11,3-Dioxolane, 2,2,4-t=imethy1- & | 4.150 & 1 & 6098 & $\mathrm{NJ} \mid$ \\
\hline 2 & |UNKNOWN & 4,435 & 1 & $2577 \mid$ & $\mathrm{NJ} \mid$ \\
\hline 3. & | UNKNOWN & 4.719 & 1 & 74331 & J1 \\
\hline 4. & | UNKNOWN & 5.878 & 1 & 27106 & $\mathbf{N J}$ \\
\hline 5. & /UNKNOWN & 6.094 & 1 & 414 & 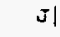 \\
\hline 16. & [ UNKNOKN & 0.212 & 1 & 4901 & $\mathrm{Jl}$ \\
\hline 17. & | Unosown & 6.792 & 1 & 14601 & Nol \\
\hline 18 & IUNKNOWN & 18.943 & 1 & 25231 & Ji \\
\hline 1 9. 544-76-3 & Hexadecane & 25.010 & 1 & 468 & $\mathrm{NJ}$; \\
\hline 110. $126-73-8$ & |Phosphoric acid, tributyl ester & | 25.834 & 1 & 554 & $\mathrm{NJ} \mid$ \\
\hline 121 & Juknown alkane & 26.927 & 1 & $1492 !$ & $\mathbf{J}$ \\
\hline 122. & Junknown alkane & 127.693 & 1 & 2971 & $\mathrm{~J} \mid$ \\
\hline 113. & Juknown alkane & 128.062 & I & 2561 & $J$ \\
\hline 114. & IUnknown alkane & 128.339 & I & 15051 & Jl \\
\hline 115. 638-36-8 & | Hexadecane, 2,6,10,14-tetramethyI- & 28.839 & 1 & 15291 & NJI \\
\hline 116. & | Unknown & | 29.337 & 1 & 4331 & J1 \\
\hline $117.0-00-0$ & | NEOPHYTADIENE & 130.045 & 1 & 7641 & $\mathrm{NJI}$ \\
\hline 18 & | Unknown alkane & 130.364 & I & 10511 & J) \\
\hline 119. & |Unknown alkane & 132.582 & 1. & 2501 & $\mathrm{Jl}$ \\
\hline 120. & | UNRTNOWN & | 35.552 & I & 511 & $\mathrm{~J}$ \\
\hline
\end{tabular}

CONCENTRATION UNITS : fug/L or $\mathrm{ug} / \mathrm{Kg}$ ) ug/ $\mathrm{Kg}$ 


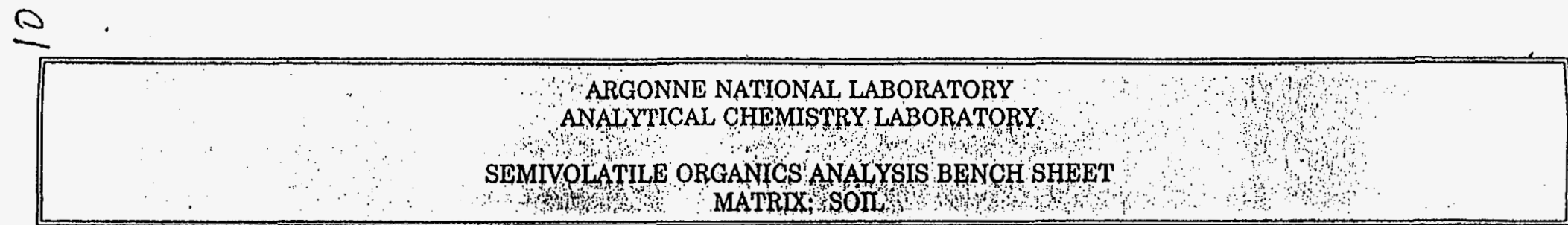

Bench Sheet Number

Sheet 1 of 1

\begin{tabular}{|c|c|c|c|c|c|c|c|c|c|c|c|c|}
\hline $\begin{array}{l}\text { ACL Sample } \\
\text { Number }\end{array}$ & $\begin{array}{c}\text { Cualoiner } \\
\text { Sample Nuniber }\end{array}$ & $\begin{array}{l}\text { Dale } \\
\text { Rec'd }\end{array}$ & $\begin{array}{l}\text { Sample } \\
\text { Size, g }\end{array}$ & $\mathrm{pH}$ & $\begin{array}{l}\text { Percent } \\
\text { Moisture }\end{array}$ & $\begin{array}{c}\text { Level } \\
\text { Low / Med }\end{array}$ & $\begin{array}{l}\text { Extraction } \\
\text { Date /'Initial }\end{array}$ & $\begin{array}{c}\text { Conc. } \\
\text { Date / Initial }\end{array}$ & $\begin{array}{c}\text { GPC } \\
\text { [YN] }\end{array}$ & $\begin{array}{l}\text { Extraction } \\
\text { Vol. A/BN }\end{array}$ & $\begin{array}{c}\text { MS } \\
\text { Anal. }\end{array}$ & Comments \\
\hline $96,-112 k^{7}$ & avelas Lab Blank & - & 30, & - & - & Med & $11 / 27 / 96^{\text {"m5 }}$ & $11 / 2.7 / a b^{15 j}$ & $N$ & $T m L$ & $\begin{array}{r}1214196 \\
1 y-r\end{array}$ & \\
\hline $97-8011.01$ & Bldg. $24-5 i$ & $10 / 23196$ & 30 & - & - & ined & $\mid 1 / 27 / 96^{m T S}$ & $11 / 274 !^{\text {ind }}$ & $N$ & $\operatorname{lm} L$ & & (J. Woscott) \\
\hline $97-8025-01$ & Bidg.24 Drain-East & $11 / 18 / 96$ & $30 \mathrm{~g}$ & - & - & thed & $11 / 27 / 96 \mathrm{mJs}^{5}$ & $11 / 27 / 96^{1+55}$ & $N$ & $1.8 \mathrm{~mL}$ & & Aliquatted ImL \\
\hline $97-8025.02$ & Bldg 24 Drain-ulest & $11 / 18196$ & 30 & - & - & Mad & $11 / 27 / 46^{\circ+15}$ & $12 / 04 / \%$ का & $N$ & $m L$ & & 7 ri . ńok \\
\hline 96.0483 .01 & 58 (Resin) & $9 / 20 / 96$ & 30 & - & - & Med & 11) 27 log $m+5$ & $12 / 0 y / 96^{\mathrm{ms}}$ & $N$ & $\ln 2$ & $\downarrow$ & (ci, sholectr) \\
\hline & & & & & & & & & & & & \\
\hline & & & & & & & & & & & & \\
\hline & & & & & & & & & & $\cdot$ & & \\
\hline & & & & & & & & & & & & \\
\hline & & & & & & & & & & & & \\
\hline & & & & & & & & & & & & \\
\hline & & & & & & & & & & & & \\
\hline & & & & & & & & & & & & \\
\hline & & & & & & & & & & & & \\
\hline
\end{tabular}

Reagent Traching

$\mathrm{MeCl}$.

$\mathrm{NaCl}_{2} \mathrm{SO}_{4}, 8024 \mathrm{KTEH}$

Standard Tracking:

$100 \mathrm{NL}\}$

_

- $\mu$ L of Base Surrogate Sud \#-

11., $\mathrm{SO}_{4}$

_ $\mu$ L. of Matrix Spike "

$A=$ Acid $=200 \mathrm{Mg} / \mathrm{m} L$ Extracts Transferred to

Y. Tsai

Dnte $12 / 4196$

MeCl/Acetone 1:1 BN549

$B+5075+B 6194$

CMT-94A (1/94) 
ANALYTICAL CHEMISTRY LABORATORY

Argonne National Laboratory

Argonne, IL 60439

REPORT OF ANALYTICAL RESULTS

Sample Material:

Liquid

Submitted by:

Charlotte Sholeen, ESH-HP, 202
Date Received:

$9 / 20 / 96$

Date Reported: $\quad$ 10/15/96

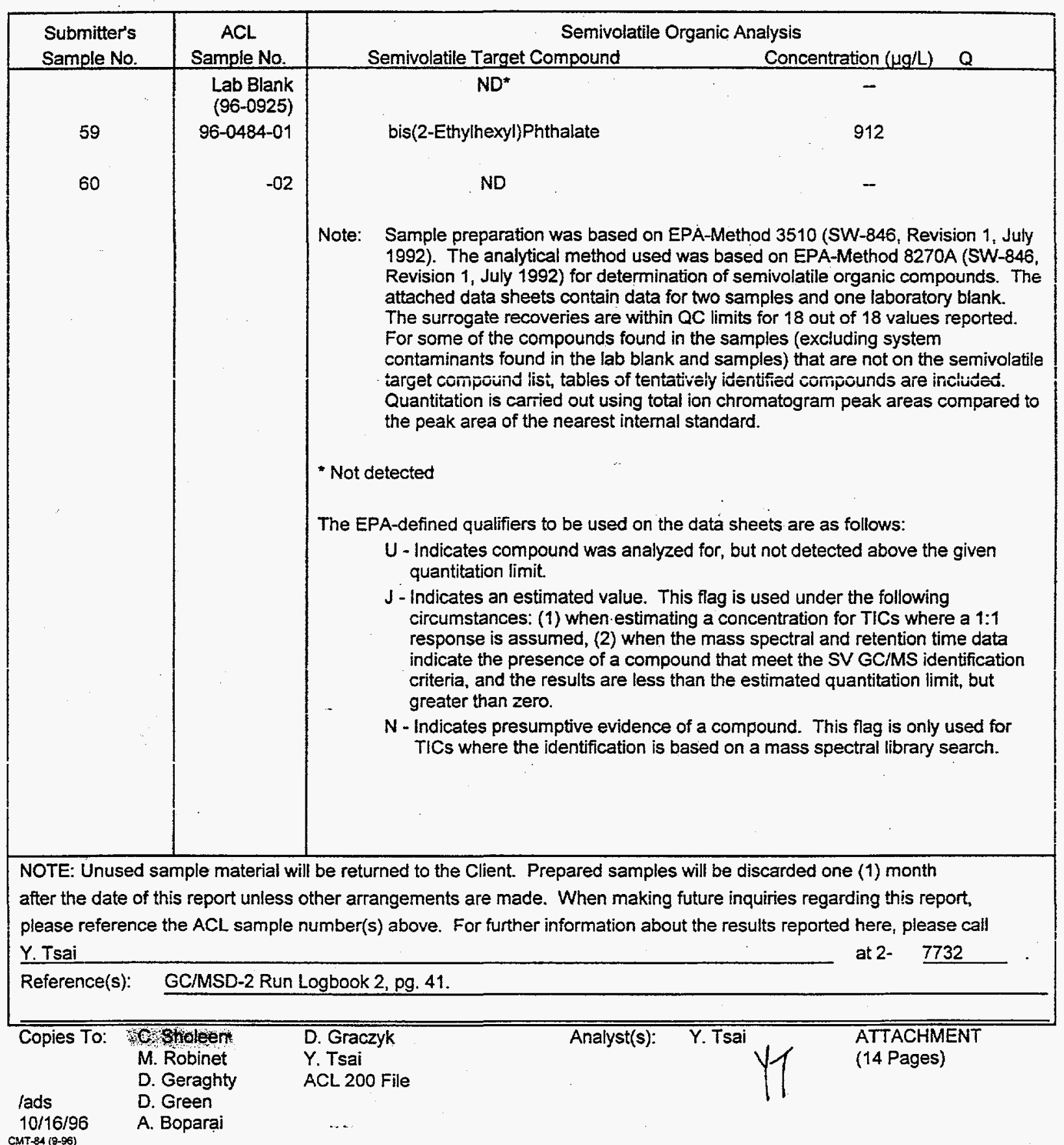


Data File: /data/msd2.i/20ct046.b/0701007.d

Report Date: 09-Oct-1996 11:18

\section{TARGET COMPOUNDS}

Client Name: C. Sholeen

Client Sample ID: lab blank, 96-0925

Sample Location:

Lab Sample ID: lab blank, 96-0925

Sample Matrix: WATER

Analysis Type: SV

Data Type: MS DATA

Misc Info:
Client SDG: 20ct046.b

Sample Date:

Sample Point:

Date Received:

Quant Type: ISTD

Level: LOW
CAS NO.

COMPOUND
CONCENTRATION UNITS:

(ug/L or ug/KG) ug/L

\begin{tabular}{|c|c|c|}
\hline 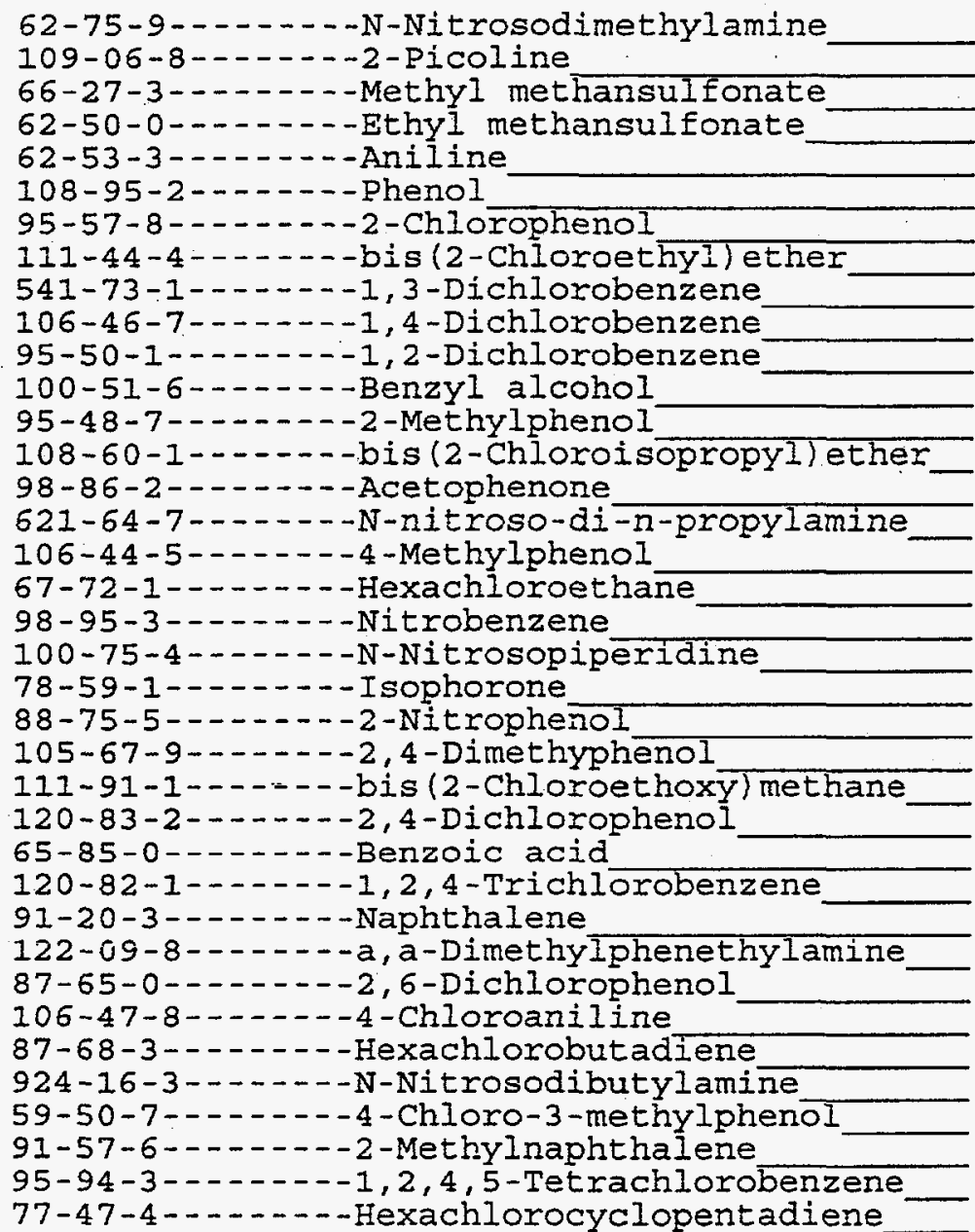 & $\begin{array}{l}100 \\
100 \\
200 \\
100 \\
100 \\
100 \\
100 \\
100 \\
100 \\
100 \\
100 \\
100 \\
100 \\
100 \\
100 \\
100 \\
100 \\
100 \\
100 \\
100 \\
100 \\
100 \\
100 \\
100 \\
100 \\
100 \\
100 \\
100 \\
200 \\
100 \\
100 \\
100 \\
100 \\
100 \\
100 \\
100 \\
200\end{array}$ & $\begin{array}{l}U \\
U \\
U \\
U \\
U \\
U \\
U \\
U \\
U \\
U \\
U \\
U \\
U \\
U \\
U \\
U \\
U \\
U \\
U \\
U \\
U \\
U \\
U \\
U \\
U \\
U \\
U \\
U \\
U \\
U \\
U \\
U \\
U \\
U \\
U \\
U \\
U\end{array}$ \\
\hline
\end{tabular}


Data File: /data/msd2.i/20ct046.b/0701007.a Report Date: 09-Oct-1996 11:18

\section{TARGET COMPOUNDS}

Client Name: $C$. Sholeen

Client Sample ID: lab blank, 96-0925

Sample Location:

Lab Sample ID: lab blank, 96-0925

Sample Matrix: WATER

Analysis Type: SV

Data Type: MS DATA

Misc Info:
Client SDG: 20ct $046 . \mathrm{b}$

Sample Date:

Sample Point:

Date Received:

Quant Type: ISTD

Level: LoW

CAS NO.

COMPOUND

CONCENTRATION UNITS:

(ug/I or $\mathrm{ug} / \mathrm{KG}) \mathrm{ug} / \mathrm{I}$

\begin{tabular}{|c|}
\hline 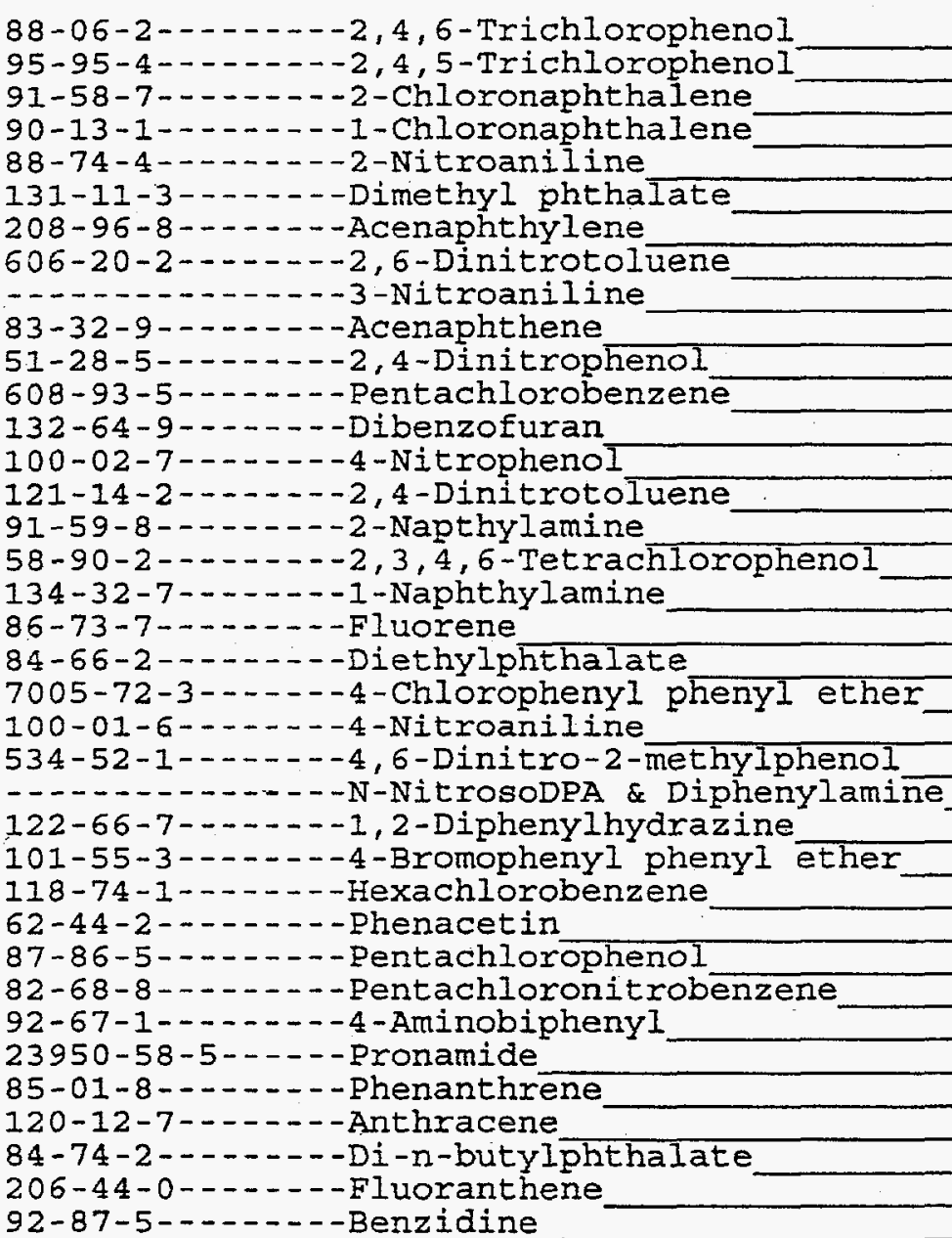 \\
\hline
\end{tabular}


Data File: /data/msd2.i/20ct046.b/0701007.d

Report Date: 09-Oct-1996 11:18

\section{TARGET COMPOUNDS}

Client Name: C. Sholeen

Client Sample ID: lab blank, 96-0925

Sample Location:

Lab Sample ID: lab blank, 96-0925

Client SDG: 20ct046.b

Sample Date:

Sample Point:

Date Received:

Sample Matrix: WATER

Analysis Type: SV

Quant Type: ISTD

Data Type: MS DATA

Level: LOW

Misc Info:

CAS NO.

COMPOUND

CONCENTRATION UNITS:

(ug/L or $\mathrm{ug} / \mathrm{KG}$ ) $\mathrm{ug} / \mathrm{L}$

Q

\begin{tabular}{|c|c|c|}
\hline 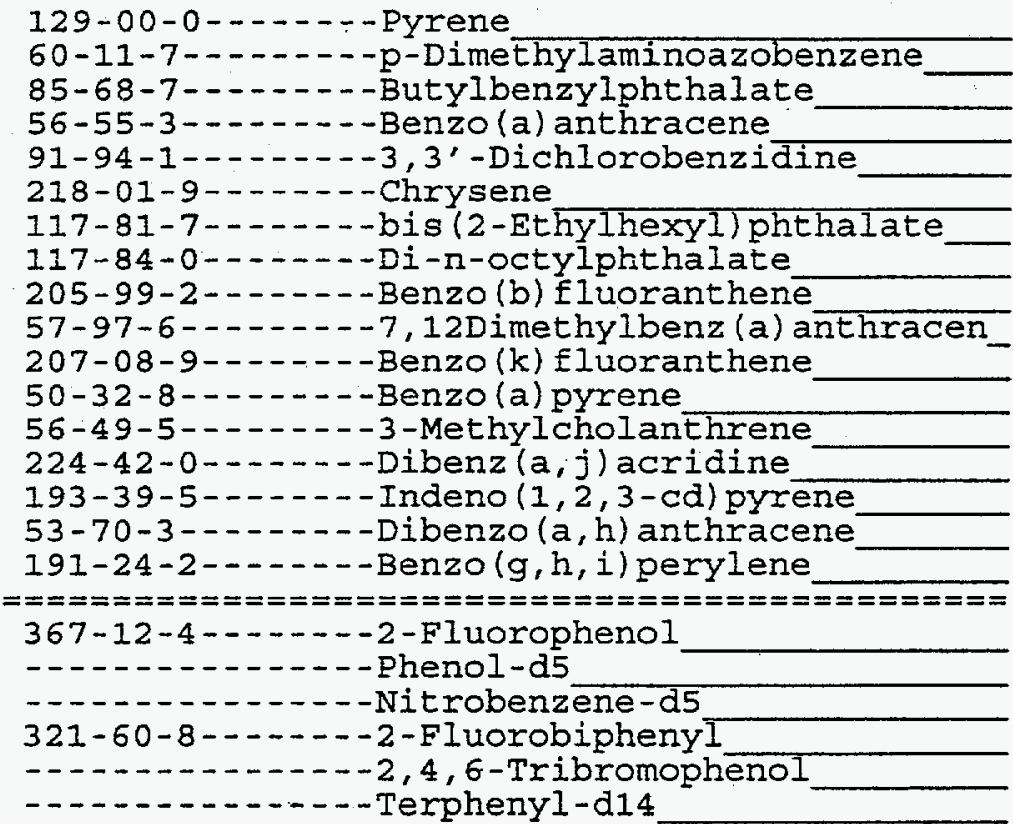 & $\begin{array}{r}100 \\
100 \\
100 \\
100 \\
100 \\
100 \\
100 \\
100 \\
100 \\
100 \\
100 \\
100 \\
100 \\
100 \\
100 \\
100 \\
100 \\
=========1 \\
1050 \\
677 \\
718 \\
646 \\
1440 \\
678\end{array}$ & $\begin{array}{c}U \\
U \\
U \\
U \\
U \\
U \\
U \\
U \\
U \\
U \\
U \\
U \\
U \\
U \\
U \\
U \\
U \\
=====\end{array}$ \\
\hline
\end{tabular}


Data File: /data/msd2.i/20ct046.b/0701007.d Report Date: 08-Oct-1996 13:18

\section{RECOVERY REPORT}

Client Name: C. Sholeen

Sample Matrix: IIQUID

Client ID: lab blank, 96-0925

Data Type: MS DATA

Spikelist File: HPMSS.spk

Method File: /data/msd2.i/20ct $046 . \mathrm{b} / \mathrm{yt} 8270 . \mathrm{m}$
Misc Info:
Client SDG: 20ct046.b

Fraction: SV

Leve1: LOW

SampleType: SAMPLE

Quant Type: ISTD

\begin{tabular}{|c|c|c|c|c|}
\hline SURROGATE COMPOUND & $\begin{array}{l}\text { AMOUNT } \\
\text { ADDED }\end{array}$ & $\begin{array}{c}\text { AMOUNT } \\
\text { RECOVERED }\end{array}$ & $\begin{array}{c}\frac{\circ}{\circ} \\
\text { RECOVERED }\end{array}$ & LIMITS \\
\hline $\begin{array}{lrl} & 4 & 2 \text {-Fluorophenol } \\
\$ & 7 & \text { Phenol-d5 } \\
\$ & 22 & \text { Nitrobenzene-d5 } \\
\$ & 45 & 2-\text { Fluorobiphenyl } \\
\$ & 70 & 2,4,6-T r i b r o m o p h e n \\
\$ & 85 & \text { Terphenyl-d14 }\end{array}$ & $\begin{array}{l}200 \\
200 \\
100 \\
100 \\
200 \\
100\end{array}$ & $\begin{array}{r}105 \\
67.7 \\
71.8 \\
64.6 \\
144 \\
67.8\end{array}$ & $\begin{array}{l}52.62 \\
33.84 \\
71.84 \\
64.58 \\
71.86 \\
67.83\end{array}$ & $\begin{array}{l}21-100 \\
10-94 \\
35-114 \\
43-116 \\
10-123 \\
33-141\end{array}$ \\
\hline
\end{tabular}

* - Values outside of oC limits

Spike Recovery: 0 out of 6 outside limits

0 out of 6 not found 
Data File: /data/msd2.i/20ct046.b/0801008.d Report Date: 09-Oct-1996 11:39

\section{TARGET COMPOUNDS}

Client Name: C. Sholeen

Client Sample ID: 59

Sample Location:

Lab Sample ID: 96-0484-01

Sample Matrix: WATER

Analysis Type: SV

Data Type: MS DATA

Misc Info:
Client SDG: 20ct046.b

Sample Date:

Sample Point:

Date Received.

Quant Type: ISTD

Level: LOW

CAS NO.

62-75-9--..--N-Nitrosodimethylamine

109-05-8-...---2-Picoline

66-27-3--.---Methyl methansulfonate

62-50-0-....-. Ethyl methansulfonate

62-53-3-...--Aniline

108-95-2.....-Phenol

95-57-8--..--2-ChIorophenol

1Il-44-4------bis (2-Chloroethyl) ether

541-73-1--.---1,3-Dichlorobenzene

106-46-7------1,4-Dichlorobenzene

95-50-1-...-.-1,2-Dichlorobenzene

100-51-6-...--Benzyl alcohol

95-48-7-2.-.-2-Methylphenol

$108-60-1-\ldots---b i s(2-C h$ loroisopropyl) ether

98-86-2-------Acetophenone

$621-64-7-\cdots---N-n i t r o s o-d i-n$-propylamine

$106-44-5---1--4-$ Methylphenol

67-72-1-...--Hexachloroethane

98-95-3-...-.-Nitrobenzene

100-75-4-..--N-Nitrosopiperidine

78-59-1--..---. Isophorone

88-75-5---.--2-Nitrophenol

105-67-9-..--2, 4-Dimethyphenol

111-91-1------bis (2-Chloroethoxy) methane

120-83-2-...--2,4-Dichlorophenol

65-85-0-.-.---Benzoic acid

120-82-1--.---1,2,4-Trichlorobenzene

91-20-3------Naphthalene

122-09-8-...--a, a-Dimethylphenethylamine

87-65-0-.....-2,6-Dichlorophenol

106-47-8-------4-Chloroaniline

87-68-3-....--Hexachlorobutadiene

924-i6-3-...--N-Nitrosodibutylamine

59-50-7--..--4-Chloro-3-methylphenol

91-57-6-------2-Methyinaphthalene

95-94-3-.-.--1,2,4,5-Tetrachlorobenzene

77-47-4-..----Hexachlorocyclopentadiene 
Data File: /data/msd2.i/20ct046.b/0801008.d Report Date: 09-Oct-1996 11:39

\section{TARGET COMPOUNDS}

Client Name: C. Sholeen

Client Sample ID: 59

Sample Location:

Lab Sample ID: 96-0484-01

Sample Matrix: WATER

Analysis Type: SV

Data Type: MS DATA

Misc Info:
Client SDG: 2oct $046 . b$

Sample Date:

Sample Point:

Date Received:

Quant Type: ISTD

Level: LOW

CAS NO.

CONCENTRATION UNITS: (ug/L or ug/KG) ug/I

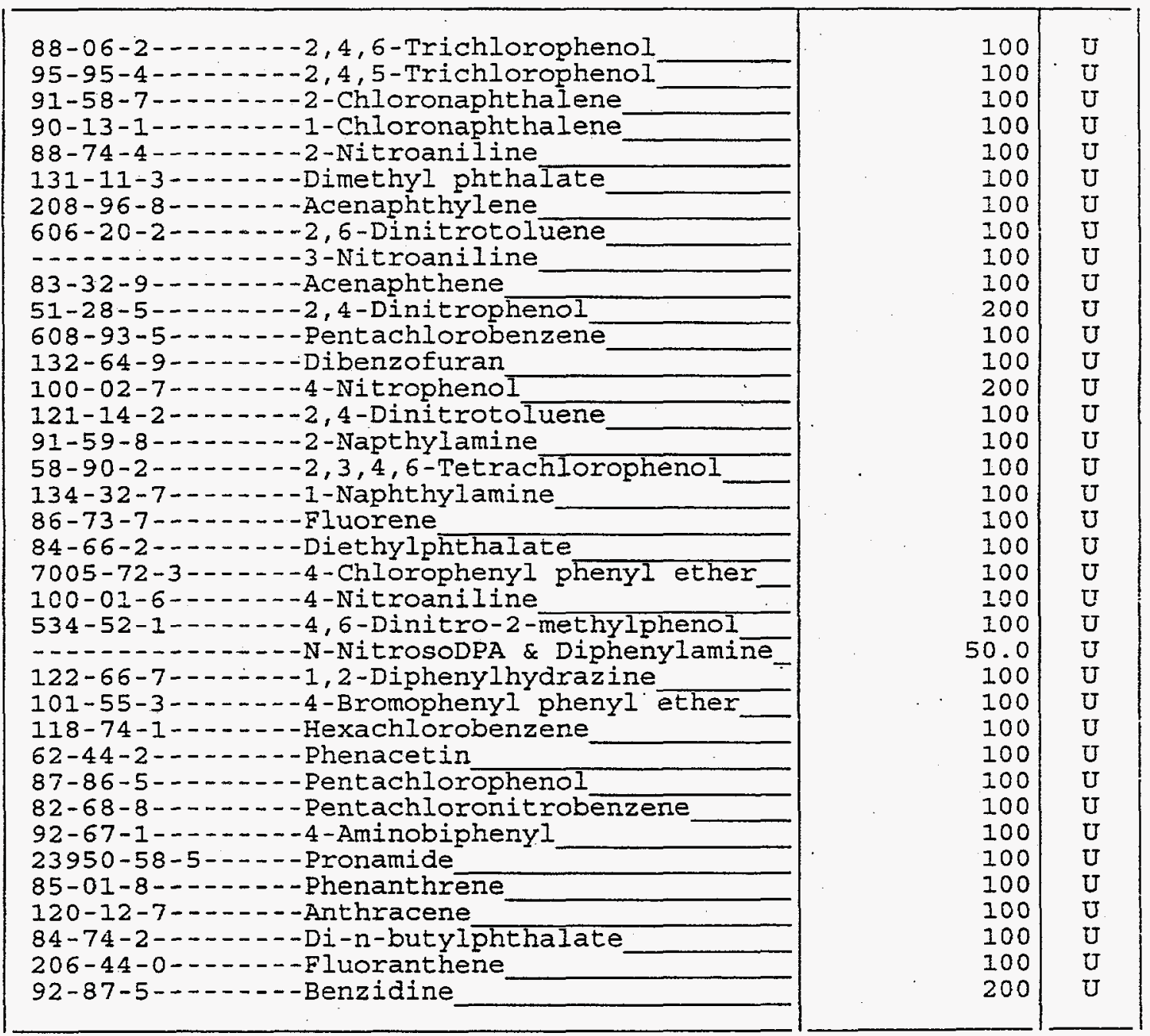


Data File: /data/msd2.i/20ct046.b/0801008.d Report Date: 09-Oct-1996 11:39

\section{TARGET COMPOUNDS}

Client Name: C. Sholeen

Client Sample ID: 59

Sample Location:

Lab Sample ID: 96-0484-01

Sample Matrix: WATER

Analysis Type: SV

Data TYpe: MS DATA

Misc Info:
Client SDG: 20ct046.b

Sample Date:

Sample Point:

Date Received:

Quant Type: ISTD

Level: LOW

CAS NO.

COMPOUND

CONCENTRATION UNITS:

(ug/L or $\mathrm{ug} / \mathrm{KG}$ ) ug/L

\begin{tabular}{|l|}
\hline $129-00-0-\cdots-1-$ \\
$60-11-7-$
\end{tabular}

\begin{tabular}{|r|c|}
100 & $\mathrm{U}$ \\
100 & $\mathrm{U}$ \\
100 & $\mathrm{U}$ \\
100 & $\mathrm{U}$ \\
100 & $\mathrm{U}$ \\
100 & $\mathrm{U}$ \\
912 & \\
100 & $\mathrm{U}$ \\
100 & $\mathrm{U}$ \\
100 & $\mathrm{U}$ \\
100 & $\mathrm{U}$ \\
100 & $\mathrm{U}$ \\
100 & $\mathrm{U}$ \\
100 & $\mathrm{U}$ \\
100 & $\mathrm{U}$ \\
100 & $\mathrm{U}$ \\
100 & $\mathrm{U}$ \\
1080 & \\
665 & \\
704 & \\
542 & \\
1670 & \\
799 & \\
& \\
\hline$=======$ &
\end{tabular}


Data File: /data/msd2.i/2oct046.b/0801008.d

Report Date: 09-Oct-1996 11:39

\section{TENTATIVELY IDENTIFIED COMPOUNDS}

Client Name: C. Sholeen

Client Sample ID: 59

Sample Location:

Lab Sample ID: 96-0484-01

Sample Matrix: WATER

Analysis Type: SV

Number TICs found: 2
Client SDG: 20ct046.b

Sample Date:

Sample Point:

Date Received:

Level: LOW

CONCENTRATION UNITS :

(ug/L or ug/KG) ug/L

\begin{tabular}{|c|c|c|c|c|}
\hline $\begin{aligned} \text { CAS NUMBER } \\
============== \\
\text { 1. } 617-84-5 \\
\text { 2. } 99-85-4\end{aligned}$ & $\begin{array}{l}\text { COMPOUND NAME } \\
======================== \\
\text { Eormamide, } N, \text { } N \text {-diethyI- } \\
\text {-gamma. - Terpinene }\end{array}$ & $\begin{array}{r}\mathrm{RT} \\
====== \\
9.218 \\
12.655\end{array}$ & $============$ & $\begin{array}{l}Q \\
==== \\
N J- \\
N J-\end{array}$ \\
\hline
\end{tabular}


Data File: /data/msd2.i/20ct046.b/0801008.d Report Date: 09-Oct-1996 11:02

\section{RECOVERY REPORT}

Client Name: C. Sholeen

Sample Matrix: LIQUID

Client ID: 59

Data Type: MS DATA

Spikelist File: HPMSS.spk

Method File: /data/msd2.i/20ct $046 . b / y t 8270 . m$
Misc Info:
Client SDG: 20ct045.b

Fraction: SV

Level: LOW

SampleTYpe: SAMPLE

Quant Type: ISTD

\begin{tabular}{|c|c|c|c|c|}
\hline SURROGATE COMPOUND & $\begin{array}{l}\text { AMOUNT } \\
\text { ADDED }\end{array}$ & $\begin{array}{c}\text { AMOUNT } \\
\text { RECOVERED }\end{array}$ & $\begin{array}{c}\frac{\circ}{\circ} \\
\text { RECOVERED }\end{array}$ & LIMITS \\
\hline $\begin{array}{lrl} & 4 & 2-\text { Fluorophenol } \\
\$ & 7 & \text { Phenol-d5 } \\
\$ & 22 & \text { Nitrobenzene-d5 } \\
\$ & 45 & 2-\text { Fluorobiphenyl } \\
\$ & 70 & 2,4,6-\text { Tribromophen } \\
\text { \$ } & 85 & \text { Terphenyl-d14 }\end{array}$ & $\begin{array}{l}200 \\
200 \\
100 \\
100 \\
200 \\
100\end{array}$ & $\begin{array}{l}108 \\
66.5 \\
70.4 \\
64.2 \\
167 \\
79.9\end{array}$ & $\begin{array}{l}54.14 \\
33.25 \\
70.46 \\
64.23 \\
83.65 \\
79.88\end{array}$ & $\begin{array}{l}21-100 \\
10-94 \\
35-114 \\
43-116 \\
10-123 \\
33-141\end{array}$ \\
\hline
\end{tabular}

* - Values outside of QC Iimits

Spike Recovery: 0 out of 6 outside limits

0 out of 6 not found 


\section{TARGET COMPOUNDS}

Client Name: C. Sholeen Client Sample ID: 60 Sample Location: Lab Sample ID: 96-0484-02 Sample Matrix: WATER Analysis Type: SV Data Type: MS DATA Misc Info:
Client SDG: 20ct046.b

Sample Date:

Sample Point:

Date Received:

Quant Type: ISTD

Level: LOW

CAS NO.

\begin{tabular}{|c|}
\hline 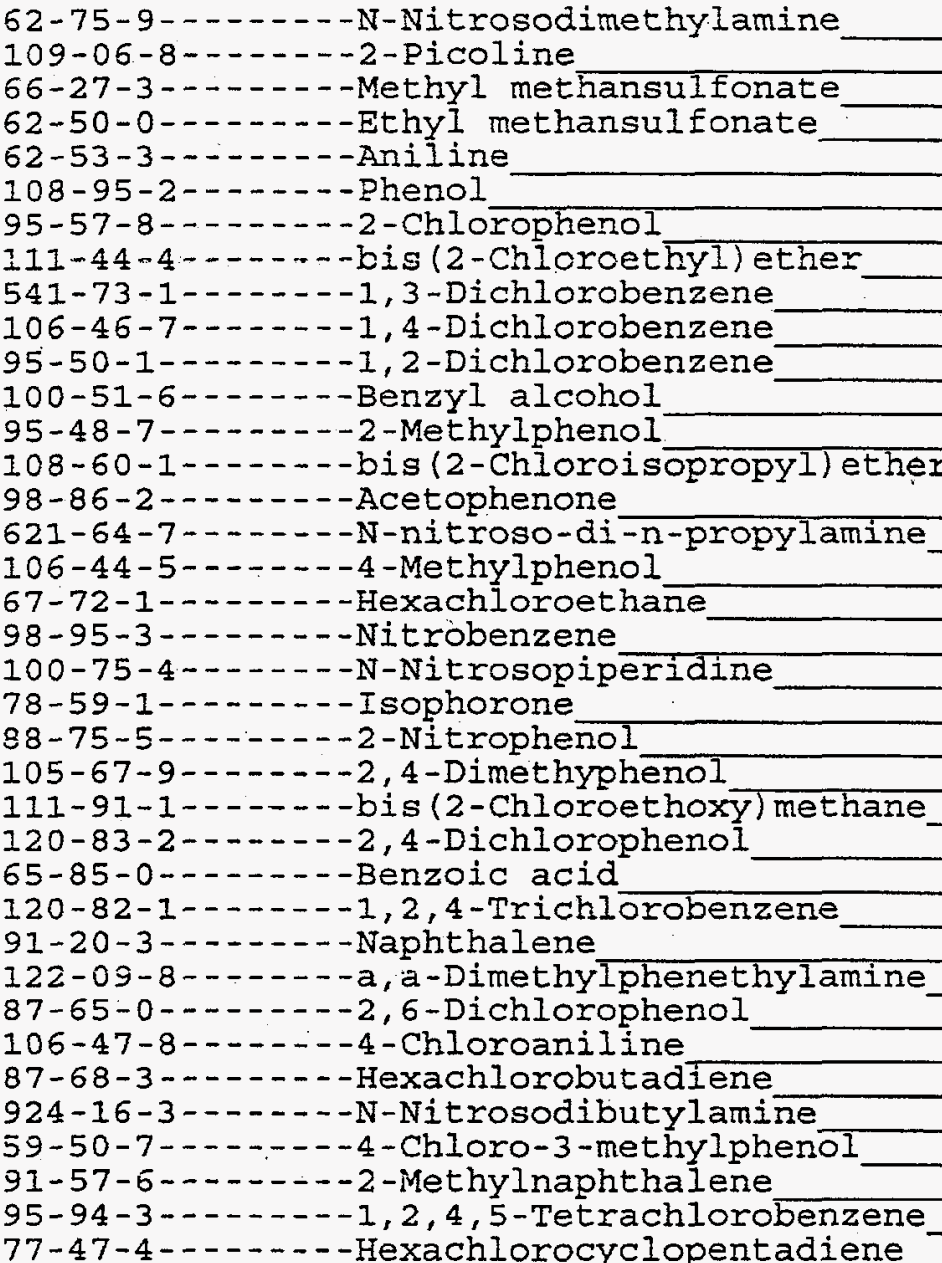 \\
\hline
\end{tabular}

\begin{tabular}{l}
$U$ \\
$U$ \\
$U$ \\
$U$ \\
$U$ \\
$U$ \\
$U$ \\
$U$ \\
$U$ \\
$U$ \\
$U$ \\
$U$ \\
$U$ \\
$U$ \\
$U$ \\
$U$ \\
$U$ \\
$U$ \\
$U$ \\
$U$ \\
$U$ \\
$U$ \\
$U$ \\
$U$ \\
$U$ \\
$U$ \\
$U$ \\
$U$ \\
$U$ \\
$U$ \\
$U$ \\
$U$ \\
$U$ \\
$U$ \\
$U$ \\
$U$ \\
$U$ \\
\hline
\end{tabular}


Data File: /data/msd2.i/20ct046.b/0901009.d Report Date: 09-Oct-1996 11:47

\section{TARGET COMPOUNDS}

Client Name: $C$. Sholeen

Client Sample ID: 60

Sample Location:

Lab Sample ID: 96-0484-02

Sample Matrix: WATER

Analysis Type: SV

Data Type: MS DATA

Misc Info:

\author{
Client SDG: 20ct $046 . \mathrm{b}$ \\ Sample Date: \\ Sample Roint: \\ Date Received: \\ Quant Type: ISTD \\ Level: LOW
}

CAS NO.

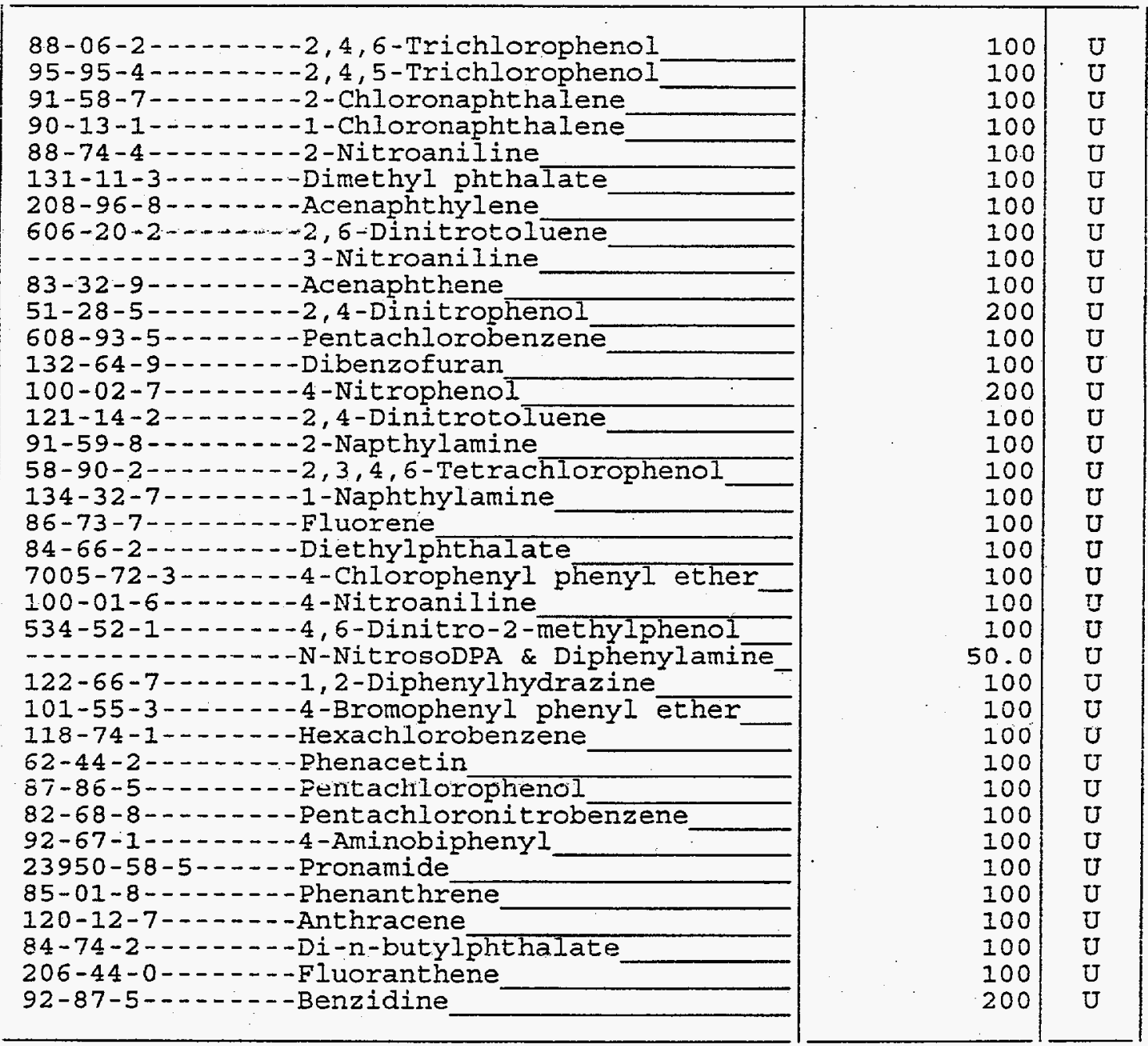


Data File: /data/msd2.i/20ct045.b/0901009.d Report Date: 09-Oct-1996 11:47

TARGET COMPOUNDS

Client Name: C. Sholeen

Client Sample ID: 60

Sample Location:

Lab Sample ID: 96-0484-02

Sample Matrix: WATER

Analysis Type: $S V$

Data Type: MS DATA

Misc Info:
Client SDG: 20ct046.b

Sample Date:

Sample Point:

Date Received:

Quant Type: ISTD

Level: LOW

CAS NO.

COMPOUND

CONCENTRATION UNITS :

( $\mathrm{ug} / \mathrm{L}$ or $\mathrm{ug} / \mathrm{KG}$ ) ug/L

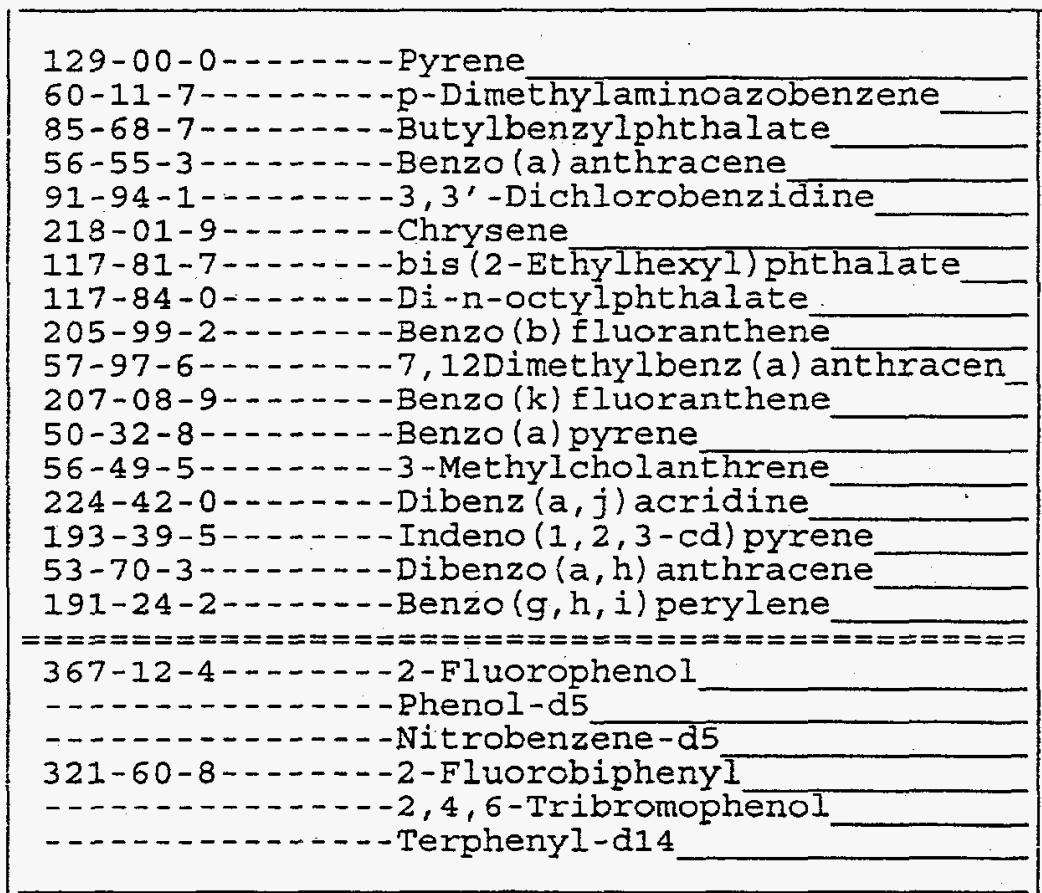

\begin{tabular}{rr|r}
100 & $\mathrm{U}$ \\
100 & $\mathrm{U}$ \\
100 & $\mathrm{U}$ \\
100 & $\mathrm{U}$ \\
100 & $\mathrm{U}$ \\
100 & $\mathrm{U}$ \\
100 & $\mathrm{U}$ \\
100 & $\mathrm{U}$ \\
100 & $\mathrm{U}$ \\
100 & $\mathrm{U}$ \\
100 & $\mathrm{U}$ \\
100 & $\mathrm{U}$ \\
100 & $\mathrm{U}$ \\
100 & $\mathrm{U}$ \\
100 & $\mathrm{U}$ \\
100 & $\mathrm{U}$ \\
100 & $\mathrm{U}$ \\
& $100=======$ \\
1170 & \\
748 & \\
847 & \\
722 & \\
1730 \\
762 \\
\end{tabular} \mid


Data File: /data/msd2.i/20ct046.b/0901009.d

\section{TENTATIVELY IDENTIFIED COMPOUNDS}

Client Name: C. Sholeen

Client Sample. ID: 50

Sample Location:

Lab Sample ID: 96-0484-02

Sample Matrix: WATER

Analysis Type: SV

Number TICs found: 2
Client SDG: 2oct046.b

Sample Date:

Sample Point:

Date Received:

Level: LOW

CONCENTRATION UNITS:

(ug/L or ug/KG) ug/L:

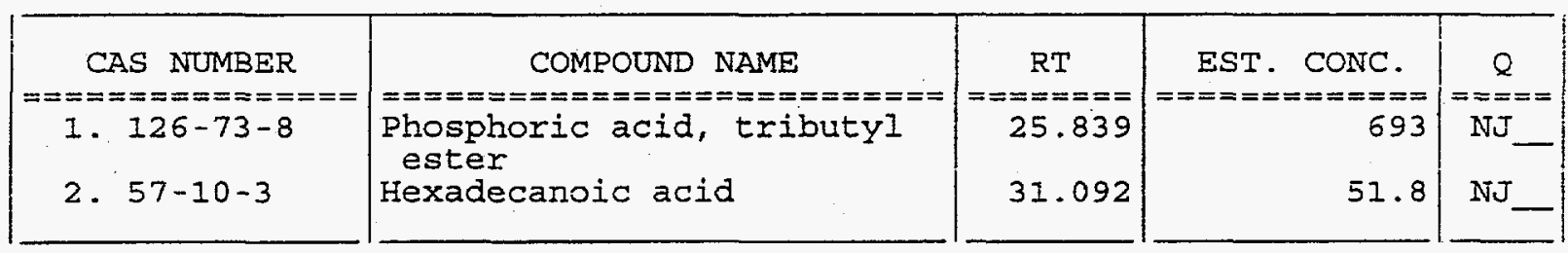


Data File: /data/msd2.i/20ct046.b/0901009.d

Report Date: 09-Oct-1996 11:03

RECOVERY REPORT

Client Name: C. Sholeen

Sample Matrix: ZIQUID

Client ID: 60

Data Type: MS DATA

Spikelist File: HPMSS spk

Method File: /data/msd2.i/20ct046.b/yt8270.m

Misc Info:

\begin{tabular}{|c|c|c|c|c|}
\hline SURROGATE COMPOUND & $\begin{array}{l}\text { AMOUNT } \\
\text { ADDED }\end{array}$ & $\begin{array}{c}\text { AMOUNT } \\
\text { RECOVERED }\end{array}$ & $\begin{array}{c}\% \\
\text { RECOVERED }\end{array}$ & LIMITS \\
\hline 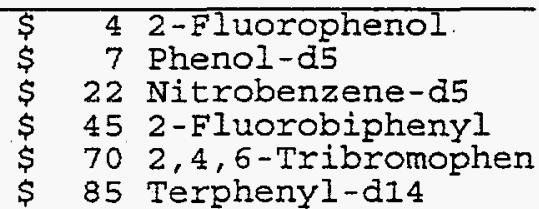 & $\begin{array}{l}200 \\
200 \\
100 \\
100 \\
200 \\
100\end{array}$ & $\begin{array}{r}117 \\
74.8 \\
84.7 \\
72.2 \\
173 \\
76.2\end{array}$ & $\begin{array}{l}58.32 \\
37.39 \\
84.72 \\
72.26 \\
86.70 \\
76.19\end{array}$ & $\begin{array}{l}21-100 \\
10-94 \\
35-114 \\
43-116 \\
10-123 \\
33-141\end{array}$ \\
\hline
\end{tabular}

* Values outside of QC limits

Spike Recovery: 0 out of 6 outside limits

0 out of 6 not found
Client SDG: 2oct046.b

Fraction: SV

Level: LOW

SampleType: SAMPLE

Quant Type: ISTD 

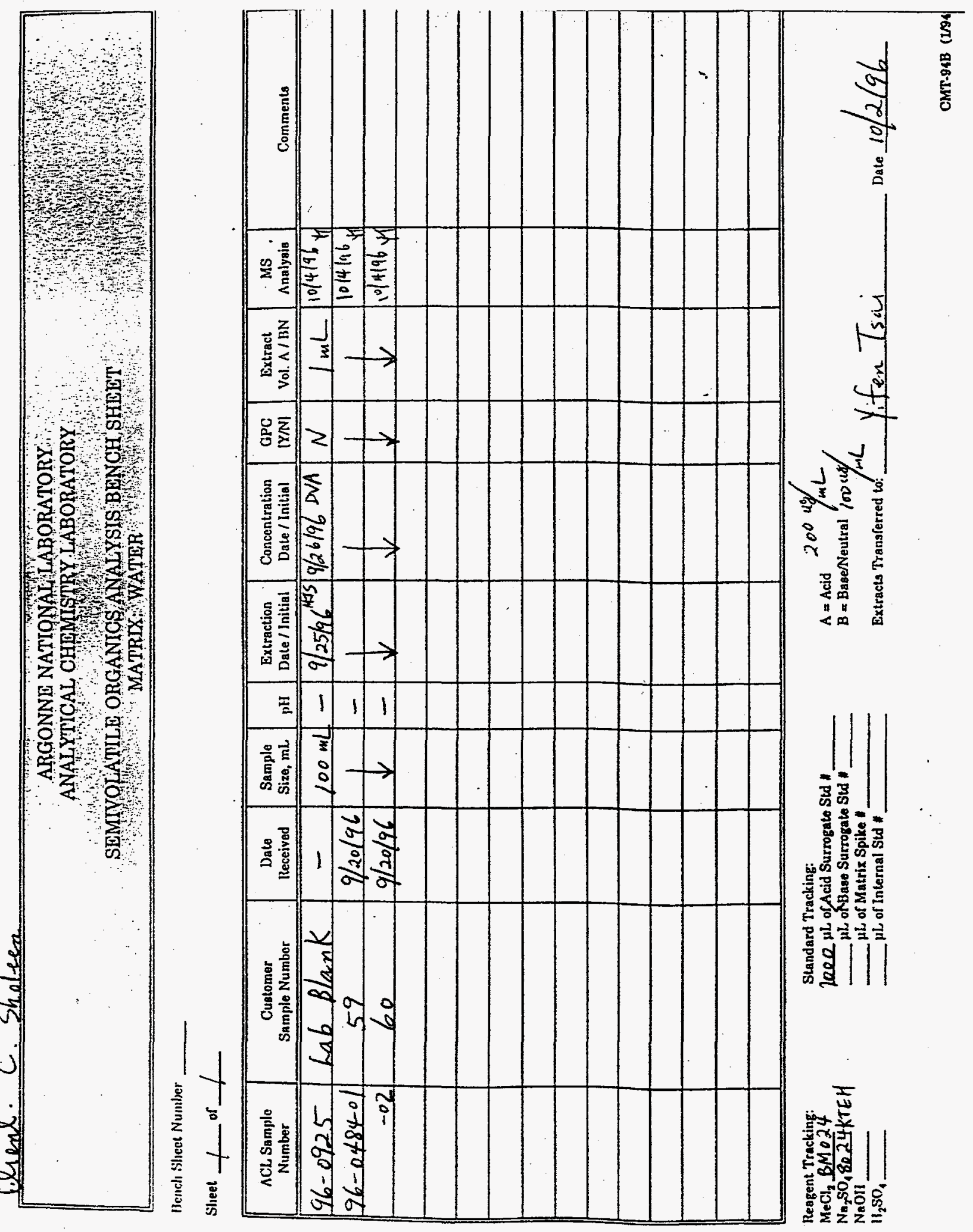
APPENDIX E: Instrument Calibration and Operational Check Records 

TABLE E1. Source Data from Tennelec for Building 579 (594)

\begin{tabular}{|c|c|c|c|c|c|c|c|c|}
\hline \multicolumn{3}{|c|}{ Real Time } & \multicolumn{2}{|c|}{ counts/min } & \multicolumn{2}{|c|}{ efficiency } & \multicolumn{2}{|c|}{$\%$ cross talk } \\
\hline & Day & Time & $\alpha$ & $\beta$ & $\alpha$ & $\beta$ & $\alpha$ to $\beta$ & $\beta$ to $\alpha$ \\
\hline THU & $09 / 19 / 96$ & $12: 59: 29$ & $13,329 \pm 82$ & $6,645 \pm 58$ & 0.240 & & 33.3 & \\
\hline THU & $09 / 19 / 96$ & $13: 01: 38$ & $5 \pm 2$ & $39,564 \pm 141$ & & 0.413 & & 0.0114 \\
\hline MON & $09 / 23 / 96$ & $15: 58: 08$ & $13,390 \pm 82$ & $6,598 \pm 57$ & 0.241 & & 33.0 & \\
\hline MON & $09 / 23 / 96$ & $16: 00: 18$ & $2 \pm 1$ & $39,380 \pm 140$ & & 0.411 & & 0.0051 \\
\hline FRI & $09 / 27 / 96$ & $09: 42: 28$ & $13,449 \pm 82$ & $6,696 \pm 58$ & 0.242 & & 33.2 & \\
\hline FRI & $09 / 27 / 96$ & $09: 44: 37$ & $3 \pm 1$ & $39,683 \pm 141$ & & 0.414 & & 0.0063 \\
\hline THU & $10 / 03 / 96$ & $12: 22: 32$ & $13,723 \pm 83$ & $6,550 \pm 57$ & 0.247 & & 32.3 & \\
\hline \multirow[t]{6}{*}{ THU } & $10 / 03 / 96$ & $12: 24: 41$ & $2 \pm 1$ & $39,457 \pm 140$ & & 0.412 & & 0.0038 \\
\hline & & Minimum & $2 \pm 1$ & $6,550 \pm 57$ & 0.240 & 0.411 & 32.3 & 0.0038 \\
\hline & & Maximum & $13,723 \pm 83$ & $39,683 \pm 141$ & 0.247 & 0.414 & 33.3 & 0.0114 \\
\hline & & Average & $6,738 \pm 42$ & $23,071 \pm 99$ & 0.242 & 0.413 & 33.0 & 0.0066 \\
\hline & Stand & Deviation & $6,736 \pm 40$ & $16,450 \pm 42$ & 0.003 & 0.001 & 0.4 & 0.0029 \\
\hline & & Count & 8 & 8 & 4 & 4 & 4 & 4 \\
\hline
\end{tabular}

The alpha source is ${ }^{241} \mathrm{Am}$

The beta source is ${ }^{90} \mathrm{Sr} /{ }^{90} \mathrm{Y}$

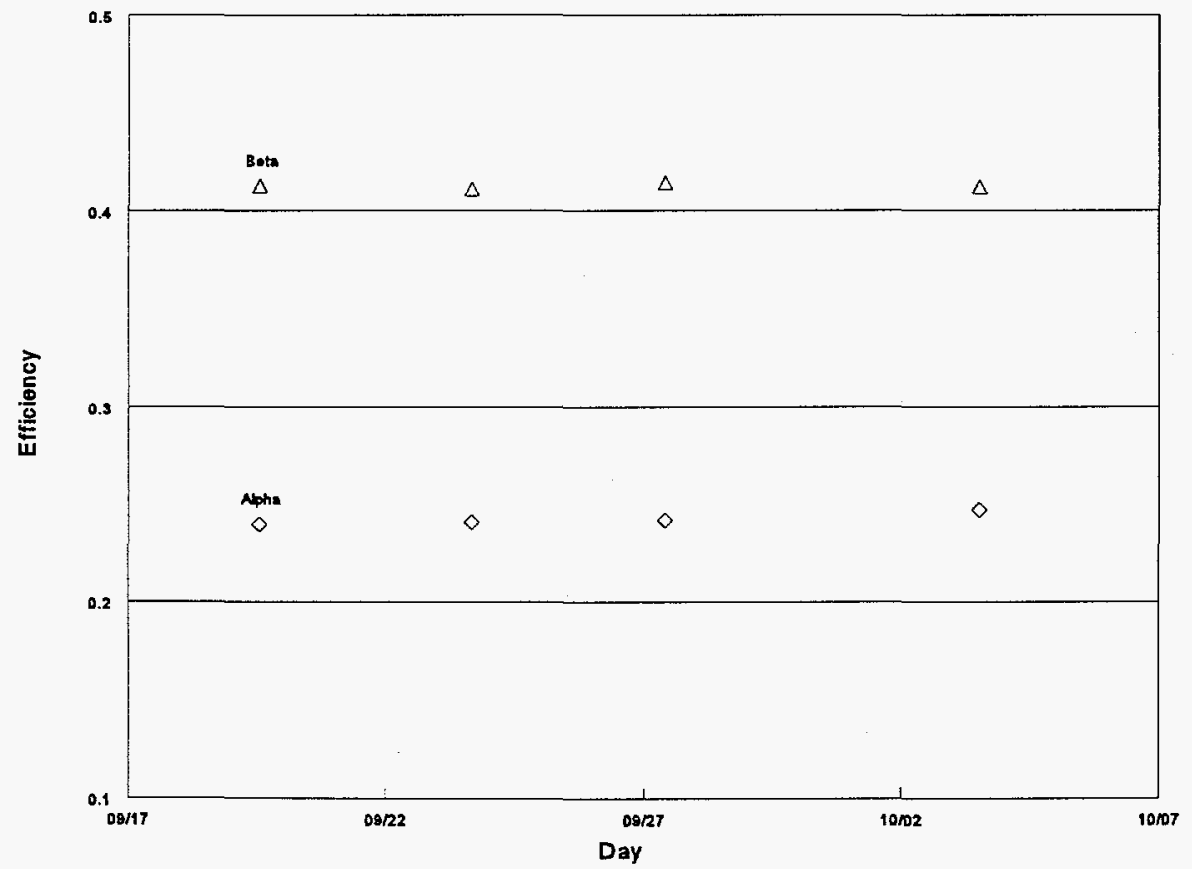

FIGURE E1. Calibration Check Data for the Tennelec System 
TABLE E2. Blank Data from Tennelec for Building 579 (594)

\begin{tabular}{|c|c|c|c|c|c|}
\hline \multicolumn{3}{|c|}{ Clock Real Time } & \multirow{2}{*}{$\begin{array}{c}\text { Count } \\
\text { Time (min) }\end{array}$} & \multicolumn{2}{|c|}{ counts/min } \\
\hline & Day & Time & & alpha & beta \\
\hline THU & 09/19/96 & $11: 47: 02$ & 200 & $0.155 \pm 0.028$ & $25.44 \pm 0.36$ \\
\hline FRI & 09/20/96 & $11: 50: 26$ & 200 & $0.155 \pm 0.028$ & $25.49 \pm 0.36$ \\
\hline MON & 09/23/96 & $12: 17: 21$ & 200 & $0.175 \pm 0.030$ & $25.55 \pm 0.36$ \\
\hline FRI & $09 / 27 / 96$ & $08: 20: 14$ & 200 & $0.160 \pm 0.028$ & $25.86 \pm 0.36$ \\
\hline \multirow[t]{6}{*}{ THU } & $10 / 03 / 96$ & $12: 20: 21$ & 200 & $0.180 \pm 0.030$ & $26.54 \pm 0.36$ \\
\hline & & & Minimum & $0.155 \pm 0.028$ & $25.44 \pm 0.36$ \\
\hline & & & Maximum & $0.180 \pm 0.030$ & $26.54 \pm 0.36$ \\
\hline & & & Average & $0.165 \pm 0.029$ & $25.77 \pm 0.36$ \\
\hline & & Star & ard Deviation & $0.010 \pm 0.001$ & $0.41 \pm 0.00$ \\
\hline & & & Count & 5 & 5 \\
\hline
\end{tabular}

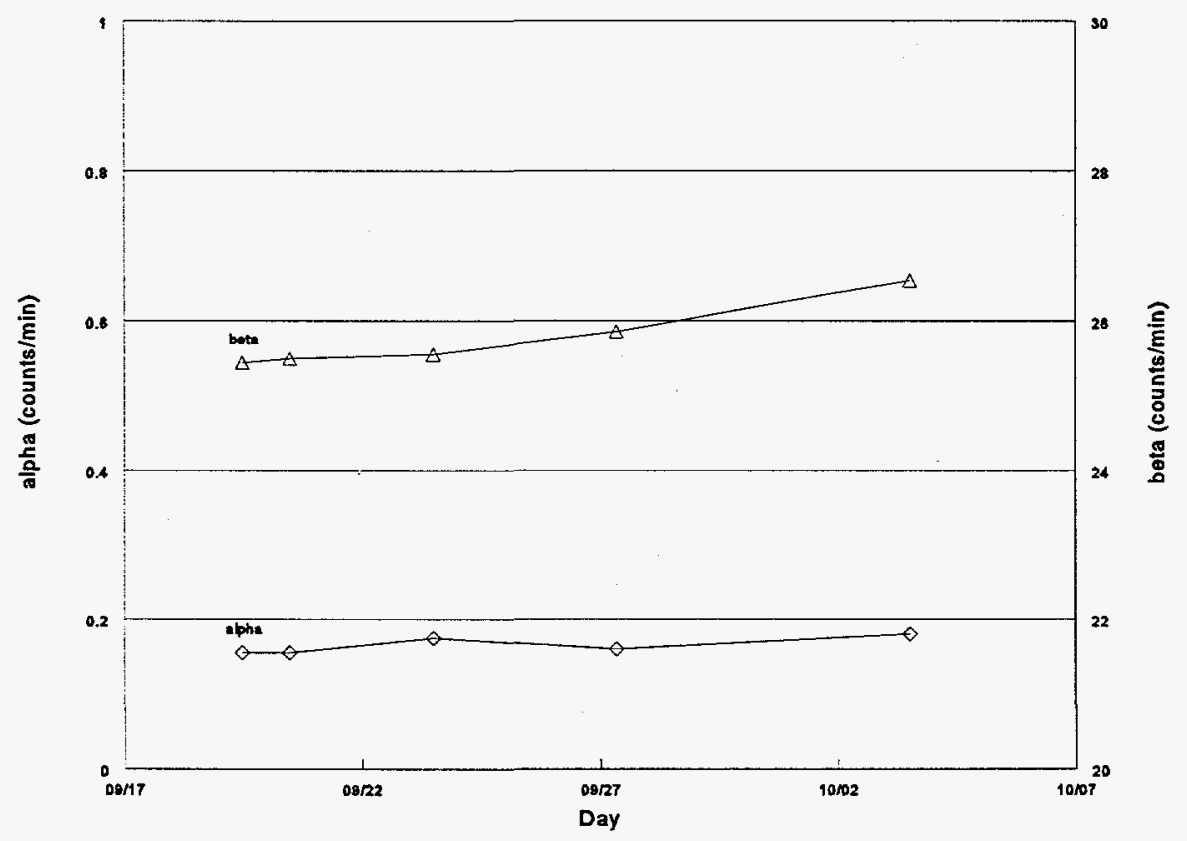

FIGURE E2. Background Check Data for the Tennelec System 


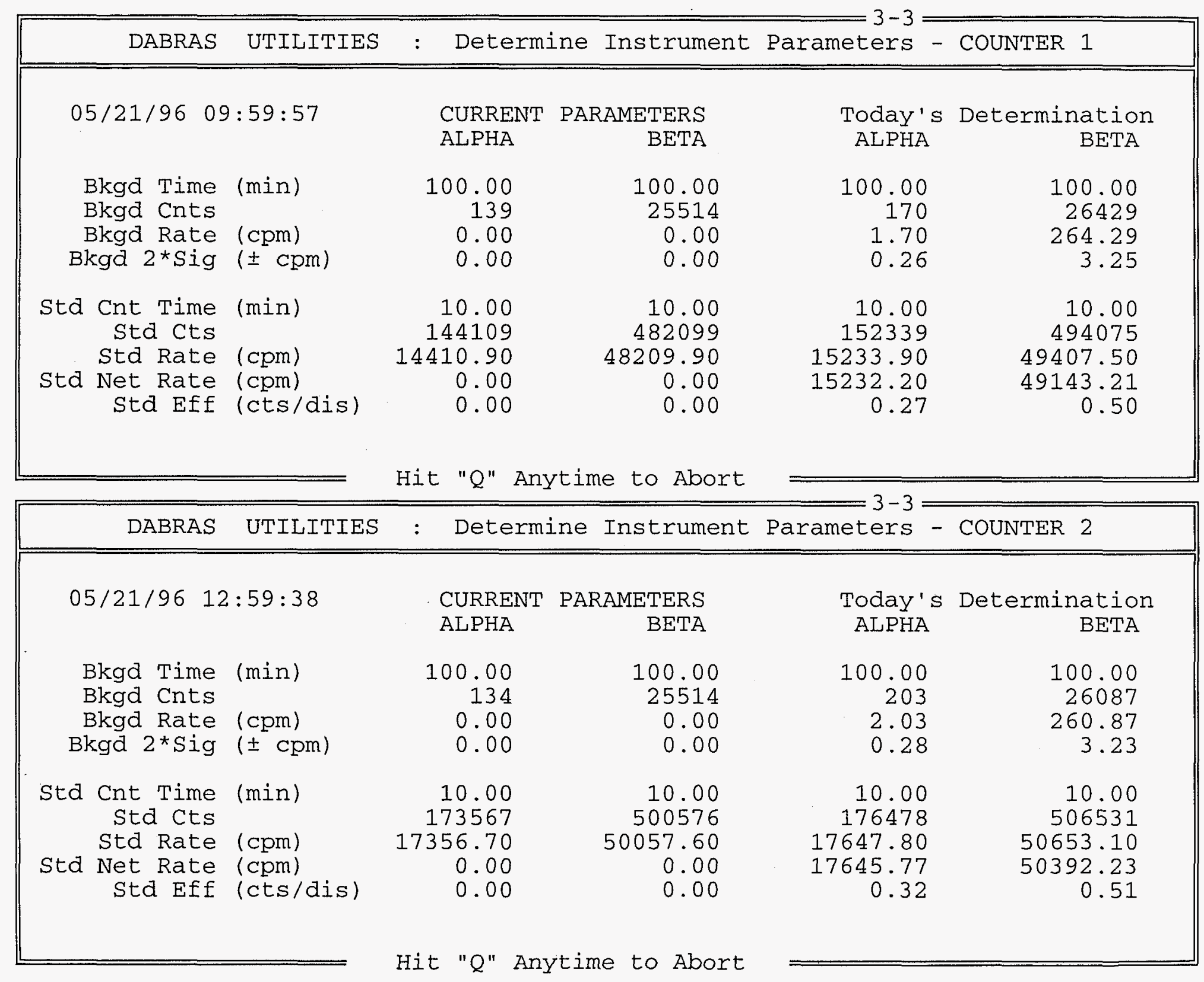




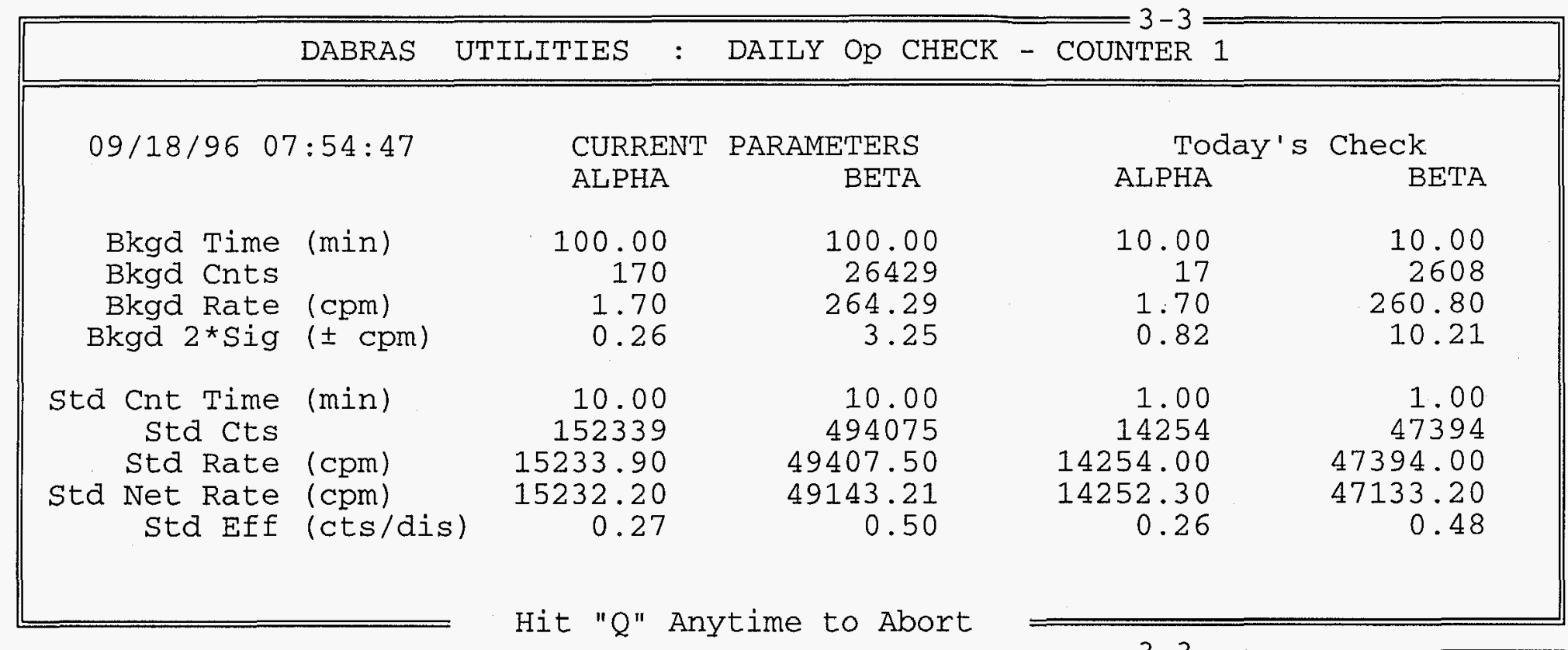

\begin{tabular}{|c|c|c|c|c|c|}
\hline & DABRAS & UTILITIES & DAILY Op CHECK & - COUNTER 2 & \\
\hline $09 / 18 / 960$ & $08: 08: 07$ & $\begin{array}{l}\text { CURRENT } \\
\text { ALPHA }\end{array}$ & $\begin{array}{r}\text { PARAMETERS } \\
\text { BETA }\end{array}$ & $\begin{array}{l}\text { Today's } \\
\text { ALPHA }\end{array}$ & $\begin{array}{l}\text { Check } \\
\text { BETA }\end{array}$ \\
\hline $\begin{array}{l}\text { Bkgd Time } \\
\text { Bkgd Cnts } \\
\text { Bkgd Rate } \\
\text { Bkgd 2* Sig }\end{array}$ & $\begin{array}{ll}e & (\min ) \\
\text { s } & \\
e & (\mathrm{cpm}) \\
g & ( \pm \mathrm{cpm})\end{array}$ & $\begin{array}{r}100.00 \\
203 \\
2.03 \\
0.28\end{array}$ & $\begin{array}{r}100.00 \\
26087 \\
260.87 \\
3.23\end{array}$ & $\begin{array}{r}10.00 \\
16 \\
1.60 \\
0.80\end{array}$ & $\begin{array}{r}10.00 \\
2550 \\
255.00 \\
10.10\end{array}$ \\
\hline $\begin{array}{r}\text { Std Cnt Time } \\
\text { Std Cts } \\
\text { Std Rate } \\
\text { Std Net Rate } \\
\text { Std Eff }\end{array}$ & $\begin{array}{ll}e & (\min ) \\
\text { s } & \\
\text { e } & (\mathrm{cpm}) \\
\text { e } & (\mathrm{cpm}) \\
\mathrm{f} & (\mathrm{cts} / \text { dis })\end{array}$ & $\begin{array}{r}10.00 \\
176478 \\
17647.80 \\
17645.77 \\
0.32\end{array}$ & $\begin{array}{r}10.00 \\
506531 \\
50653.10 \\
50392.23 \\
0.51\end{array}$ & $\begin{array}{r}1.00 \\
15080 \\
15080.00 \\
15078.40 \\
0.27\end{array}$ & $\begin{array}{r}1.00 \\
48186 \\
48186.00 \\
47931.00 \\
0.49\end{array}$ \\
\hline
\end{tabular}




\begin{tabular}{|c|c|c|c|c|c|}
\hline & DABRAS & UTILITIES & DAILY OP CHECK & - COUNTER 1 & \\
\hline $09 / 19 / 96 \quad 0^{\prime}$ & $7: 53: 24$ & $\begin{array}{l}\text { CURRENT } \\
\text { ALPHA }\end{array}$ & $\begin{array}{r}\text { PARAMETERS } \\
\text { BETA }\end{array}$ & $\begin{array}{l}\text { Today's } \\
\text { ALPHA }\end{array}$ & $\begin{array}{l}\text { Check } \\
\text { BETA }\end{array}$ \\
\hline $\begin{array}{l}\text { Bkgd Time } \\
\text { Bkgd Cnts } \\
\text { Bkgd Rate } \\
\text { Bkgd 2*Sig }\end{array}$ & $\begin{array}{ll} & (\mathrm{min}) \\
& (\mathrm{cpm}) \\
& ( \pm \mathrm{cpm})\end{array}$ & $\begin{array}{r}100.00 \\
170 \\
1.70 \\
0.26\end{array}$ & $\begin{array}{r}100.00 \\
26429 \\
264.29 \\
3.25\end{array}$ & $\begin{array}{r}10.00 \\
57 \\
5.70 \\
1.51\end{array}$ & $\begin{array}{r}10.00 \\
2683 \\
268.30 \\
10.36\end{array}$ \\
\hline $\begin{array}{r}\text { Sta Cnt Time } \\
\text { sta cts } \\
\text { std Rate } \\
\text { std Net Rate } \\
\text { std Eff }\end{array}$ & $\begin{array}{l}=(\min ) \\
=(\mathrm{cpm}) \\
=(\mathrm{cpm}) \\
=(\mathrm{cts} / \text { dis })\end{array}$ & $\begin{array}{r}10.00 \\
152339 \\
15233.90 \\
15232.20 \\
0.27\end{array}$ & $\begin{array}{r}10.00 \\
494075 \\
49407.50 \\
49143.21 \\
0.50\end{array}$ & $\begin{array}{r}1.00 \\
14542 \\
14542.00 \\
14536.30 \\
0.26\end{array}$ & $\begin{array}{r}1.00 \\
47858 \\
47858.00 \\
47589.70 \\
0.48\end{array}$ \\
\hline
\end{tabular}

\begin{tabular}{|c|c|c|c|c|c|}
\hline & DABRAS & UTILITIES & DAILY OP CHECK & - COUNTER 2 & \\
\hline $09 / 19 / 960$ & $08: 15: 03$ & $\begin{array}{l}\text { CURRENT } \\
\text { ALPHA }\end{array}$ & $\begin{array}{r}\text { PARAMETERS } \\
\text { BETA }\end{array}$ & $\begin{array}{l}\text { Today's } \\
\text { ALPHA }\end{array}$ & $\begin{array}{l}\text { Check } \\
\text { BETA }\end{array}$ \\
\hline $\begin{array}{l}\text { Bkgd Time } \\
\text { Bkgd Cnts } \\
\text { Bkgd Rate } \\
\text { Bkgd 2*Sig }\end{array}$ & $\begin{array}{ll}e & (\min ) \\
s & \\
e & (\mathrm{cpm}) \\
g & ( \pm \mathrm{cpm})\end{array}$ & $\begin{array}{r}100.00 \\
203 \\
2.03 \\
0.28\end{array}$ & $\begin{array}{r}100.00 \\
26087 \\
260.87 \\
3.23\end{array}$ & $\begin{array}{r}10.00 \\
39 \\
3.90 \\
1.25\end{array}$ & $\begin{array}{r}10.00 \\
2666 \\
266.60 \\
10.33\end{array}$ \\
\hline $\begin{array}{l}\text { Std Cnt Time } \\
\text { std Cts } \\
\text { Std Rate } \\
\text { std Net Rate } \\
\text { std Eff }\end{array}$ & $\begin{array}{ll}e & (\min ) \\
\text { s } & \\
\text { e } & (c p m) \\
e & (c p m) \\
\text { f } & \text { (cts/dis) }\end{array}$ & $\begin{array}{r}10.00 \\
176478 \\
17647.80 \\
17645.77 \\
0.32\end{array}$ & $\begin{array}{r}10.00 \\
506531 \\
50653.10 \\
50392.23 \\
0.51\end{array}$ & $\begin{array}{r}1.00 \\
14928 \\
14928.00 \\
14924.10 \\
0.27\end{array}$ & $\begin{array}{r}1.00 \\
48115 \\
48115.00 \\
47848.40 \\
0.48\end{array}$ \\
\hline
\end{tabular}




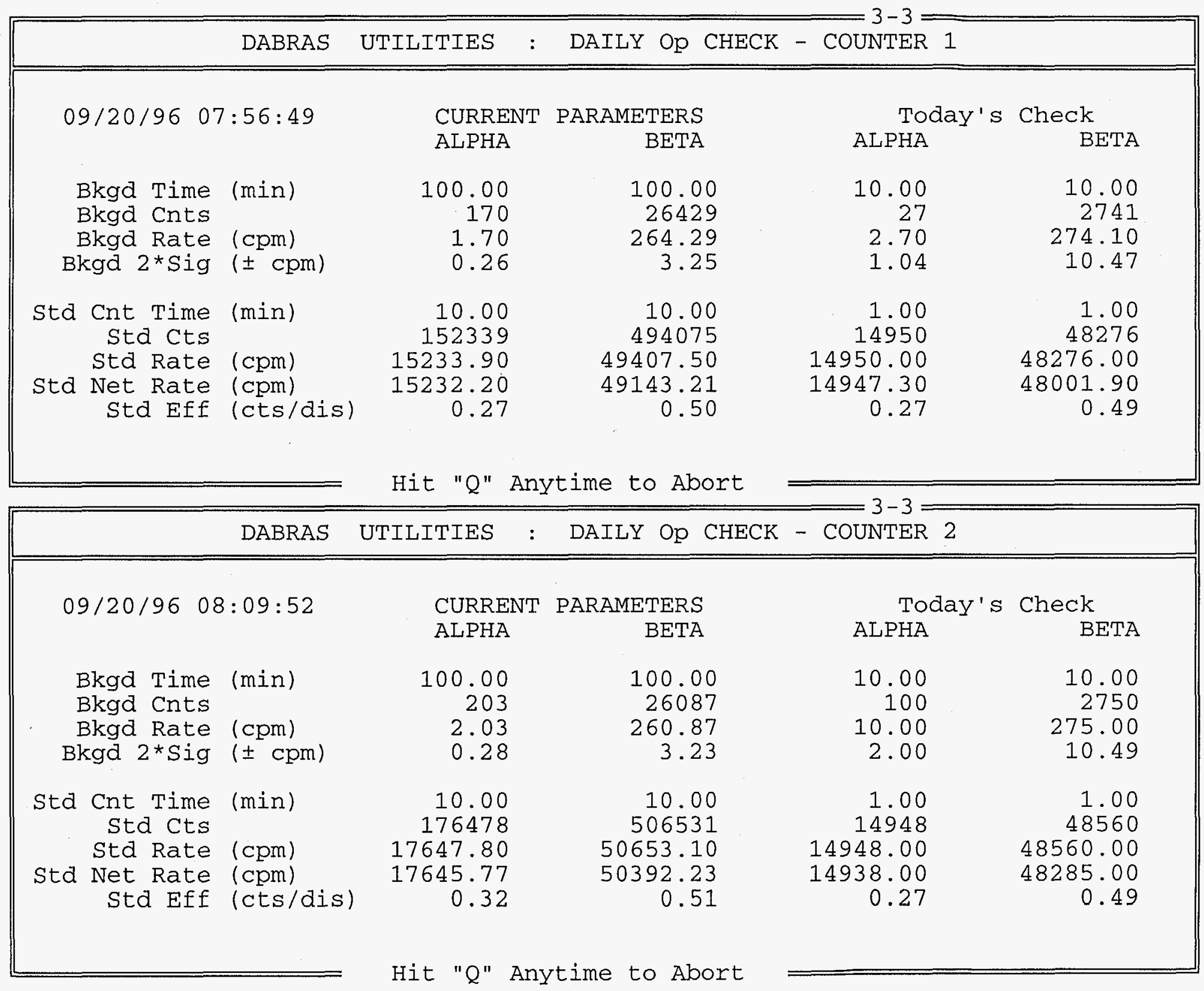




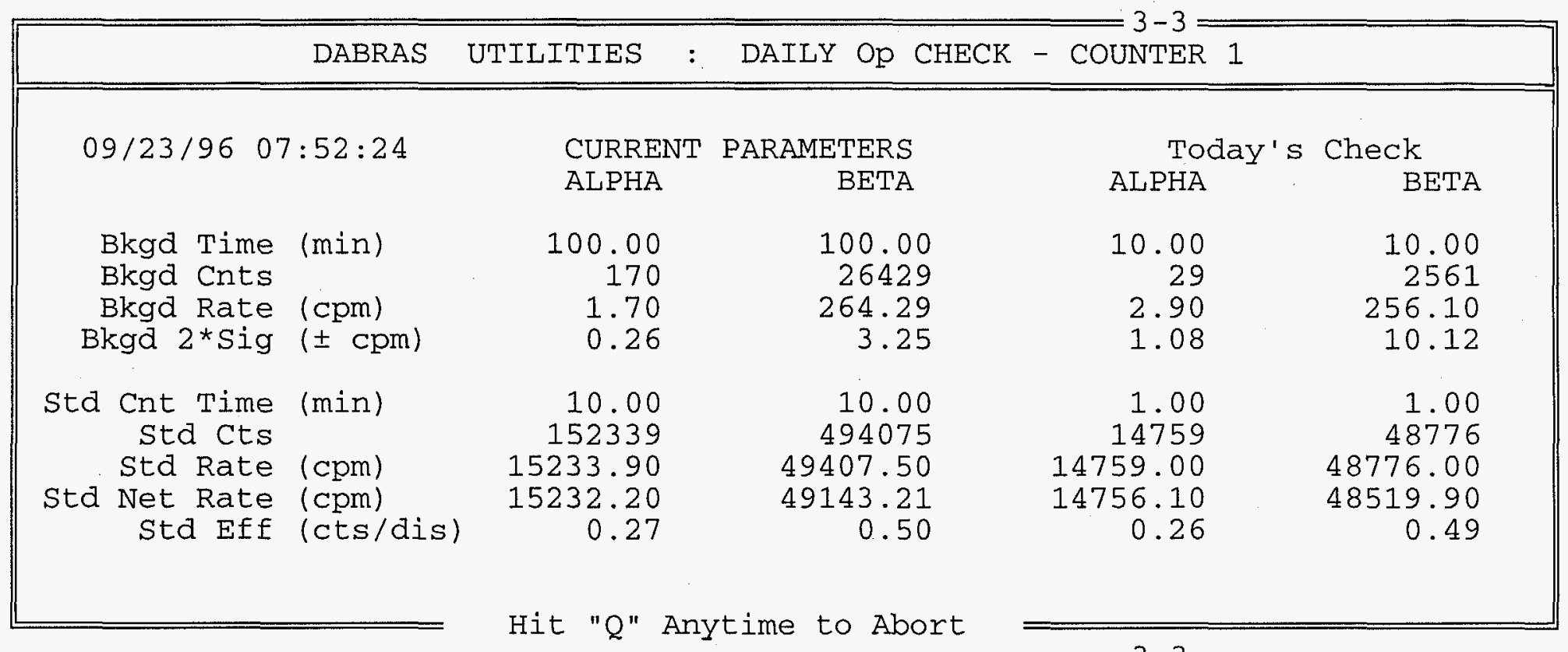

\begin{tabular}{|c|c|c|c|c|c|}
\hline & DABRAS & UTILITIES & DAILY Op CHECK & - COUNTER 2 & \\
\hline $09 / 23 / 960$ & $08: 06: 12$ & $\begin{array}{l}\text { CURRENT } \\
\text { ALPHA }\end{array}$ & $\begin{array}{r}\text { PARAMETERS } \\
\text { BETA }\end{array}$ & $\begin{array}{l}\text { Today's } \\
\text { ALPHA }\end{array}$ & $\begin{array}{l}\text { Check } \\
\text { BETA }\end{array}$ \\
\hline $\begin{array}{l}\text { Bkgd Time } \\
\text { Bkgd Cnts } \\
\text { Bkgd Rate } \\
\text { Bkgd 2*Sig }\end{array}$ & $\begin{array}{ll}e & (\min ) \\
s & \\
g & (\mathrm{cpm}) \\
g & ( \pm \mathrm{cpm})\end{array}$ & $\begin{array}{r}100.00 \\
203 \\
2.03 \\
0.28\end{array}$ & $\begin{array}{r}100.00 \\
26087 \\
260.87 \\
3.23\end{array}$ & $\begin{array}{r}10.00 \\
81 \\
8.10 \\
1.80\end{array}$ & $\begin{array}{r}10.00 \\
2750 \\
275.00 \\
10.49\end{array}$ \\
\hline $\begin{array}{l}\text { std Cnt Time } \\
\text { Std Cts } \\
\text { std Rate } \\
\text { std Net Rate } \\
\text { Std Eff }\end{array}$ & $\begin{array}{l}\text { (min) } \\
\text { (cpm) } \\
\text { (cpm) } \\
\text { (cts/dis) }\end{array}$ & $\begin{array}{r}10.00 \\
176478 \\
17647.80 \\
17645.77 \\
0.32\end{array}$ & $\begin{array}{r}10.00 \\
506531 \\
50653.10 \\
50392.23 \\
0.51\end{array}$ & $\begin{array}{r}1.00 \\
15170 \\
15170.00 \\
15161.90 \\
0.27\end{array}$ & $\begin{array}{r}1.00 \\
48751 \\
48751.00 \\
48476.00 \\
0.49\end{array}$ \\
\hline
\end{tabular}




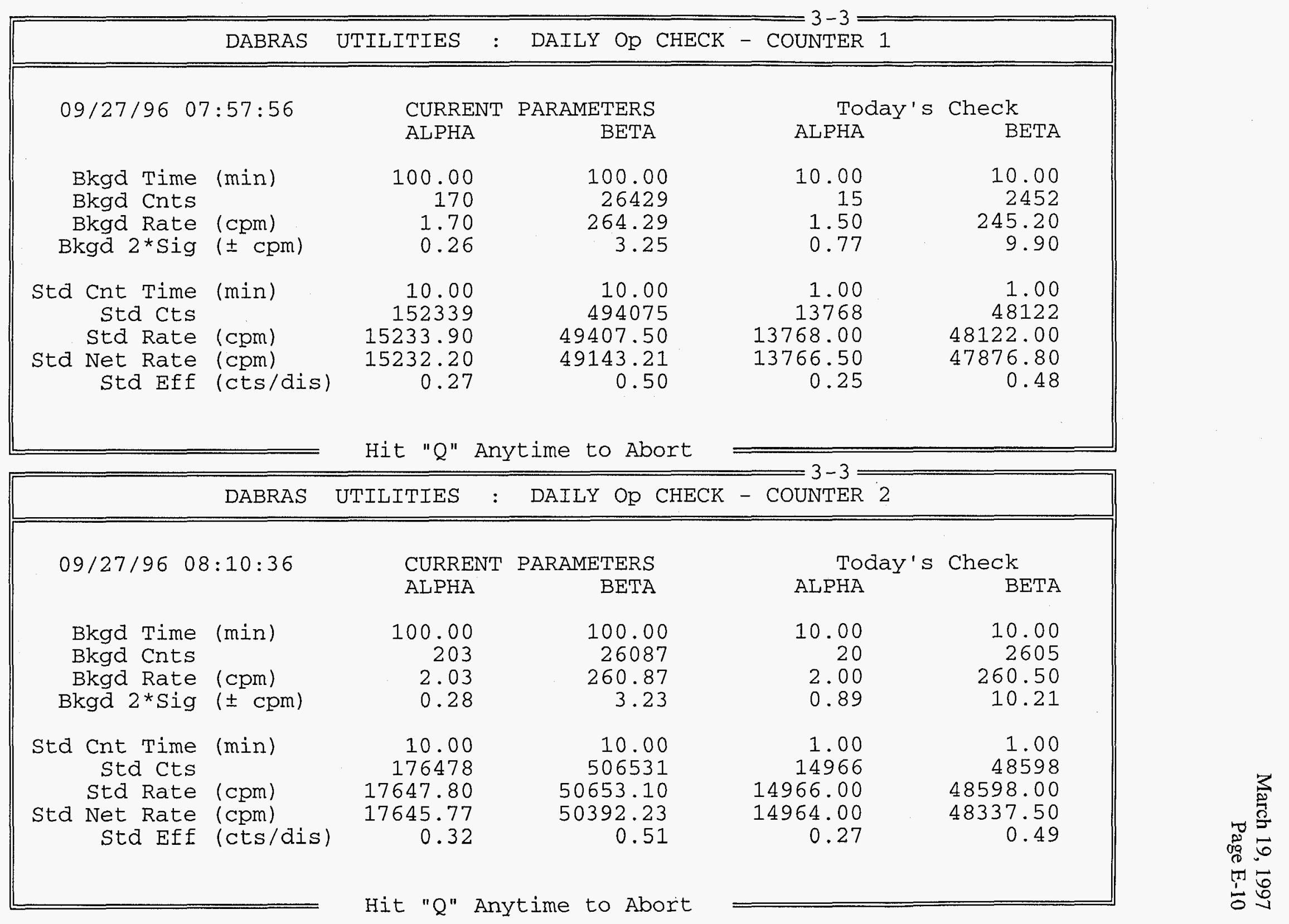


Certificate \#015-D2

rev. 1.0, January 1996

\section{INSTROMEATT MAINTENANCE/CALIBRATION CERTIFICATE}

Set\#: 2259 Procedure\#: 015 Configuration: surface contam Type: $\mathbf{x}$ Dual Scintillator (dpm units) Unit\#:5497 MEr/Model: NE Technology Electra Unit\#: 69 C) Mfr/Model: NE Technology DP6A

$S / N$ : $\mathrm{S} / \mathrm{N}:$ $S / N 5 \%$

Pulse Generator, Eberline MPElectrostatic Kilovoltmeter: Alpha Source: ESO $2 k$ Alpha source: $\frac{4 m 24}{50}$, Activity 60800 dpm

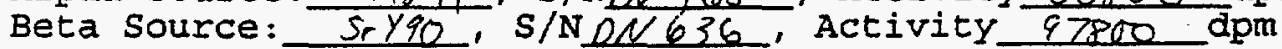

\section{I) MAINTEMANCE/PRECAIIBRATION:}

Window: Cabling:_ Mechanical/Cleanliness : Battery Voltage (Parameter \# 0 ): 4.5 (ref: > 4) Threshold: 22 (verify $25 \mathrm{mv}$ with the Minipulser) As the Electra "supervisor" set the Upper Level Discriminator (Parameter \#6): $2 \mathrm{~V}$ (ref: $2.00 \mathrm{~V}$ ), then INHIBIT:Count Rate Check @ $\overline{10 \mathrm{k}} \mathrm{cpm}$ : HV Calib.: (compare parameter \#3

w/ the Electrostatic)

High Voltage Adjustment:

Using Sr-90 beta source, observe count rates in both alpha and beta channels. Adjust HV so that count rate in alpha channel is less than $0.1 \%$ of count rate in beta channel. Beta cpm: $273 \mathrm{~K}$ Alpha cpm: $1 /$ (e.g., $\leq . / \%$ of beta) $\mathrm{HV}=$ 990 , and INHIBIT:

\section{Parameter settings:}

As the Electra "supervisor" (i.e., the internal switch s12 to $O N$ ); set the remaining parameters as follows:

Parameter \#4 (Overload Current): $10 \mu \mathrm{A}$, then INHIBIT:

Parameter \#5 (Deadtime): $3 \mu \mathrm{sec}$, then INHIBIT:

Parameter \#8 (Units): cpm

Parameter \#A (inhibit bkgd subt): set to OFF

parameter \#b (inhibit integrate): set to OFF

Parameter \#C (rate mode): set to preset

Parameter \#d (preset response time): set to 3 sec

Parameter \#E (pulse mode): set to dUAL

Parameter \#F (ohms): set to $\mathrm{s} 66$

II) PRIMARY CAIIBRATION:

Response to Alpha Std: $2.2 \mathrm{Kcpm}$. 200 efficiency (alpha) Alpha Mode Bkgd: $5 \mathrm{cpm}$ (ref.: $<7 \mathrm{cpm}$ )

Response to Beta Std: $275 \mathrm{~K}$ cpm; $279^{\circ}$ efficiency (beta)

Beta Mode Bkgd: 360 cpm (ref.: $<400 \mathrm{cpm}$ )

Integrate Check:_— Audible Functional Check:

Parameter \#8 (Units): change from cpm to dpm

Parameter \#9 (Efficiencies): enter efficiencies from above Now set switch SI-2 back to OFF (user), and leave switch S1-3 set to on (hide)

REMARTS :

Calibrated by: Date: $5,1-96$ 


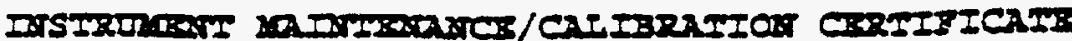

Ser\#:14/ Procedure\#: 018 Configuration: Scintillator Type: ${ }^{2}-2$ maI

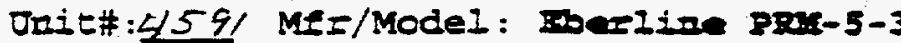

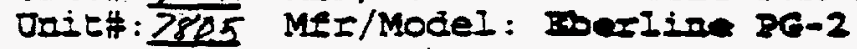

$S / N: 2983$

Pulse Generator, Bberline MPBlectrostatic Rilovol tmeter:

Source (s) : Pu-239, S/N 7552

Am-24I,, $5 / N 7551$

J-235 , S/N 22B6102

$S / N:$

$5 / 2300$

ACEIVIEY $4150000 \mathrm{dpm}$ $5 / N \quad 250556$

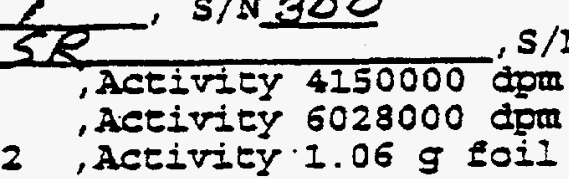

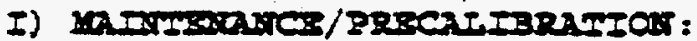

Batteries: OK Cabling: Of Mechanical/Cleaninness: lavi I\{ mreshold: id wV (Eixed at 5 to $10 \mathrm{mV}$ ).

Window: $12.5 \mathrm{mV}=2.5 \%$ (ref.: 25t of Threshold mV)

Audible Function check: ase

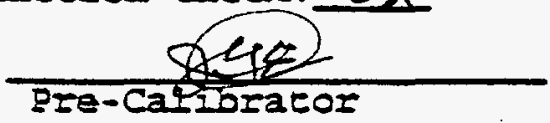

$\frac{5 / 52}{\text { Date }}$

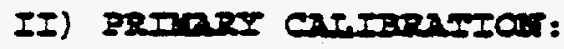

\begin{tabular}{|c|c|c|c|c|}
\hline $\begin{array}{c}\text { Range } \\
\text { (cpin) } \\
\frac{2.3}{8.3}\end{array}$ & 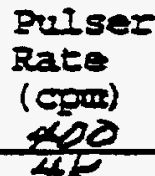 & $\begin{array}{l}\text { As } \\
\text { Roued } \\
\text { (cout) } \\
3>5\end{array}$ & $\begin{array}{l}\text { As } \\
\text { Left } \\
\text { (cpins) }\end{array}$ & $\begin{array}{l}\text { f Dife. } \\
\text { Puiser vs } \\
\text { As Left } \\
\text { uso }\end{array}$ \\
\hline$\frac{x-3}{e-4}$ & $\frac{4 k}{40 K}$ & $\frac{3.6 k}{39 k}$ & $\frac{x}{4}$ & $\frac{4 k}{40 k}$ \\
\hline$x-1$ & 400K & $380 k$ & $43 k$ & SOOK \\
\hline
\end{tabular}

With IEA in, adjust EV to maximize detector response to isocope of interest:

Isotope As zound As Left
Mode (cpm)
(cpm)

$$
\text { Isotope }
$$

(If Applicable)

EV- 1

IV- 2

Pu-239

Am-24I

J-235

$\frac{13 k}{300 k} \frac{\frac{14 k}{3 \Delta 0 K}}{175 K}$

$N / A$

$N / A$

$N / A$

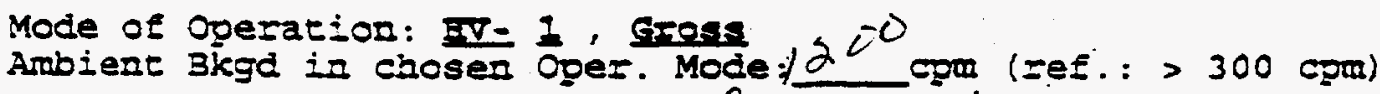

REDares:

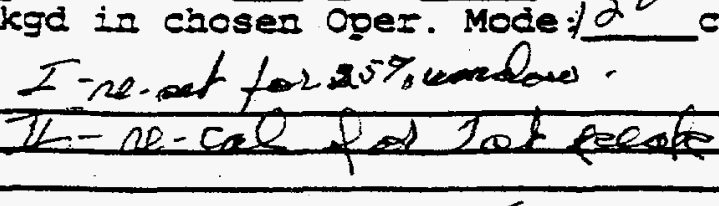

$\frac{2 \cdot x+1}{\text { Pamary-carabracor }}$

15,96

Dace 
NE Instrument Operational Check Log

\begin{tabular}{|c|c|c|c|c|c|}
\hline Building 579 & & & Location & & \\
\hline SOURCE \# & $E_{A} 119$ & MINIMUM & 099333 & Br 80516 & \\
\hline & DN 639 & MAXIMUM & $a 68000$ & $\beta Y 120750$ & \\
\hline INSTRUMENT SET \# & DATE & A PASS & RG PASS & IMITIIe & cOMAMratc \\
\hline 1257 & $9 / 16 / 96$ & 2 & 2 & $D C 6$ & \\
\hline 1259 & $9 / 17 / 96$ & - & - & $D C$ & \\
\hline 1259 & $9 / 18 / 96$ & $\sigma$ & 一 & $D C C$ & \\
\hline 1259 & $9 / 19 / 96$ & $\longleftarrow$ & 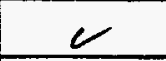 & $0 c 6$ & \\
\hline 1259 & $9 / 20 / 96$ & - & 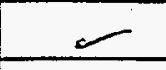 & Dec & \\
\hline 1259 & $9 / 2-3 / 96$ & 2 & $r$ & DCS & \\
\hline 1259 & $9 / 25 / 96$ & J & 7 & $O C C$ & \\
\hline & & & & & \\
\hline & & & & & \\
\hline & & & & & \\
\hline & & & & & \\
\hline & & & & & \\
\hline & & & & & \\
\hline & & & & & \\
\hline & & & & & \\
\hline & & & & & \\
\hline & & & & & \\
\hline & & & & & \\
\hline & & & & & \\
\hline & & & & & \\
\hline & & & & & \\
\hline & & & & & \\
\hline & & & & & \\
\hline & & & & & \\
\hline & & & & & \\
\hline & & & & & \\
\hline & & & & & \\
\hline & & & & & \\
\hline
\end{tabular}


PRM 5-3 Operational Check Log

\begin{tabular}{|c|c|c|c|c|}
\hline Building 579 & & Location & & \\
\hline SOURCE \# Mande & MINIMUM & 90.0 & cts/min & \\
\hline & MAXIMUM & 12000 & $\mathrm{cts} / \mathrm{min}$ & \\
\hline & & & & \\
\hline INSTRUMENT SET \# & DATE & PASS/READING & INITIALS & COMMENTS \\
\hline$|\psi|$ & $9 / 17 / 96$ & -8000 & $x$ & \\
\hline 141 & $9 / 18 / 96$ & -9000 & $D C C$ & \\
\hline 41 & $9 / 20 / 96$ & -8000 & $D C 6$ & \\
\hline & & & & \\
\hline & & & & \\
\hline & & & & \\
\hline & & & & \\
\hline & & & & \\
\hline & & & & \\
\hline & & & & \\
\hline & & & & \\
\hline & & & & \\
\hline & & & & \\
\hline & & & & \\
\hline & & & & \\
\hline & & & & \\
\hline & & & & \\
\hline & & & & \\
\hline & & & & \\
\hline & & & & \\
\hline & & & & \\
\hline & & & & \\
\hline & & & & \\
\hline & & & & \\
\hline & & & & \\
\hline & & & & \\
\hline & & & & \\
\hline & & & & \\
\hline
\end{tabular}


Internal

N.L. Contos (ESH-HP)

D.C. Geraghty (ESH-HP)

M.J. Robinet (ESH-HP)

C.M. Sholeen (ESH-HP)

HP Office - 13 copies (ESH)

C. Grandy (EMO)

J. Johnson (EMO)

D.C. Pancake (EMO)

T. Yule (TD)

R.W. Rose (TD) - 9 copies

TIS File (203)

External

DOE-OSTI (2)

ANL-E Library (Bldg 203)

ANL-W Library (J. Krieger IS/AW - 752)

DOE CH Operations Office 